\title{
Keratin Networks in Live Cells
}

\author{
Dissertation \\ for the award of the degree \\ "Doctor rerum naturalium" \\ of the Georg-August-Universität Göttingen
}

within the doctoral program

Göttingen Graduate School for Neurosciences, Biophysics, and Molecular Biosciences (GGNB)

of the Georg-August University School of Science (GAUSS)

submitted by

\section{Jens-Friedrich Nolting}

from Hannover

Göttingen 2014 


\title{
Thesis committee:
}

\author{
Prof. Dr. Sarah Köster \\ Institute for X-Ray Physics \\ Georg-August-Universität Göttingen \\ Prof. Dr. Andreas Janshoff \\ Institute for Physical Chemistry \\ Georg-August-Universität Göttingen \\ Prof. Dr. Tim Salditt \\ Institute for X-Ray Physics \\ Georg-August-Universität Göttingen
}




\title{
Members of the examination board:
}

\author{
Reviewer: Prof. Dr. Sarah Köster
}

Institute for X-Ray Physics

Georg-August-Universität Göttingen

Second reviewer: Prof. Dr. Andreas Janshoff

Institute for Physical Chemistry

Georg-August-Universität Göttingen

\section{Further members of the examination board:}

Prof. Dr. Tim Salditt

Institute for X-Ray Physics

Georg-August-Universität Göttingen

Dr. Florian Rehfeldt

Third Institute of Physics

Georg-August-Universität Göttingen

Ph.D. Thomas Burg

Department for Biological Micro- and Nanotechnology

Max Planck Institute for Biophysical Chemistry, Göttingen

Prof. Dr. Jörg Enderlein

Third Institute of Physics

Georg-August-Universität Göttingen

\section{Date of oral examination:}





\section{Contents}

1 Introduction 1

2 State of the Art 3

2.1 Cytoskeleton . . . . . . . . . . . . . . . . . . . 3

2.2 Keratin - an Intermediate Filament Protein . . . . . . . . . . . 4

$2.2 .1 \quad$ Aggregation of Keratin Filaments $\ldots \ldots \ldots . . \ldots .6$

2.2 .2 Mechanical Properties of Keratin . . . . . . . . . . . . . 9

2.2 .3 Keratin in Cells . . . . . . . . . . . . . . . . . . . . . 9 9

2.2.4 The Role of Keratin in the Cellular Response to Stress . . . . . . 12

2.3 Buckling Events in Cells . . . . . . . . . . . . . . . . . 13

3 Materials and Methods 17

3.1 Cell Culture . . . . . . . . . . . . . . . . . . . . . . . 17

3.2 Microscopy Methods . . . . . . . . . . . . . . . . . . . . 18

$3.2 .1 \quad$ Fluorescence Microscopy . . . . . . . . . . . . . . . . . . 18

3.2 .2 Confocal Microscopy . . . . . . . . . . . . . . . . . . 20

3.3 Experiments with Cells under Shear-Flow . . . . . . . . . . . 22

3.4 Microfluidic Devices . . . . . . . . . . . . . . . . . . . 23

3.5 FEM Simulation . . . . . . . . . . . . . . . . . . . . 25

3.6 Buckling-Experiments . . . . . . . . . . . . . . . 26

4 Cells in Micro-Flow 27

$4.1 \quad$ Fluid Dynamics. . . . . . . . . . . . . . . . . . . . . . . . . . . . . . 27

4.1 .1 Theoretical Background . . . . . . . . . . . . . . . . 28

4.1 .2 Flow-Fields in Microfluidic Channels. . . . . . . . . . . . . 30

4.2 Data Analysis of Keratin Networks in Cells . . . . . . . . . . . 33

4.2 .1 Image Preprocessing . . . . . . . . . . . . . . . . 34

4.2 .2 Image-to-Image Cross-Correlations . . . . . . . . . . . . 35

4.2 .3 Characterization of Node-Trajectories . . . . . . . . . . 37 
4.3 Reduced Keratin Network Motion under Flow . . . . . . . . . . . . . 41

4.3 .1 Global Approach: 2D-Cross-Correlation . . . . . . . . . . . . . 41

4.3 .2 Local Effects of Shear Flow on Keratin Networks . . . . . . . . 43

4.4 Treatment with Blebbistatin . . . . . . . . . . . . . . . 48

4.5 Discussion . . . . . . . . . . . . . . . . . 50

5 Buckling of Keratin Bundles 59

5.1 Theoretical Background . . . . . . . . . . . . . . . . . 59

$5.1 .1 \quad$ Euler Buckling and Bending Energy . . . . . . . . . . . 60

5.1 .2 Energy Contribution due to External Forces. . . . . . . . . . 65

5.1 .3 Additional Energy Contribution for Embedded Rods . . . . . . . 66

5.1 .4 Constrained Buckling . . . . . . . . . . . . . . . . . 69

5.1 .5 Bending of Coupled-Rods . . . . . . . . . . . . 71

5.1 .6 Constrained Buckling of Bundles . . . . . . . . . . 73

5.2 Evaluation of Buckling Events . . . . . . . . . . . . . . . . 73

5.2 .1 Determination of the Buckling Wavelength . . . . . . . 74

5.2 .2 Analysis of the Bundle Diameter . . . . . . . . . . 79

5.3 Results . . . . . . . . . . . . . . . . . . . . . 8 82

5.3 .1 Structure of Keratin Bundles . . . . . . . . . . . . . . 82

5.3 .2 Buckling of Keratin Bundles . . . . . . . . . . . . . . . 85

5.4 Discussion . . . . . . . . . . . . . . . . . . . . 88

5.4 .1 Influence of the Surrounding Matrix . . . . . . . . . . 88

5.4 .2 Strong Coupling between Filaments inside a Bundle . . . . . . 90

5.4 .3 Theoretical Model . . . . . . . . . . . . . . . . . . . . 93

5.4 .4 Buckling in Sheared Cells . . . . . . . . . . . . . . . 94

5.4 .5 Possible Optimizations . . . . . . . . . . . . . . . . 96

6 Summary and Conclusion 99

\begin{tabular}{ll}
\hline Appendix A Additional Datasets & 103
\end{tabular}

\begin{tabular}{ll}
\hline Appendix B TEM Preparation & 109
\end{tabular}

\begin{tabular}{ll}
\hline List of Figures & 111
\end{tabular}

\begin{tabular}{ll}
\hline List of Abbreviations and Symbols & 115
\end{tabular} 
Bibliography

List of Publications

133

Lebenslauf

135

\begin{tabular}{ll}
\hline Danksagung & 137
\end{tabular} 


\section{Introduction}

The building blocks of higher forms of life, including for example vertebrates, are cells. Eukaryotic cells contain a variety of constituents like organelles, DNA and a large diversity of proteins. These proteins are large biological macromolecules which fulfill multiple and crucial tasks within a cell. The spectrum of their functions ranges from the specific catalysis of chemical reactions over signal transduction and force generation up to structure-giving functions. The group of proteins for the latter tasks covers networks of stiff structures which substantially determine the mechanical properties of the cell, as well as binding proteins which cross-link various cell constituents.

One structural protein is keratin. It is an intermediate filament protein which tends to form higher-order aggregates that ultimately result in bundles of filaments. Apart from representing the most prominent constituent in varied forms of hair or nails, keratin is also a key structural protein in the outer layer of epithelial tissue, which covers all inner and outer surfaces throughout the body of at least vertebrates. In epithelial cells, keratin helps to withstand potentially harmful mechanical forces and to guarantee the integrity of the cell. The importance of keratin becomes most evident in regard to the broad spectrum of diseases which are related to keratin mutations and malfunctions. One example of such a disease is epidermolysis bullosa simplex, which results in the formation of blisters in the skin of affected persons under the influence of even small mechanical shear forces.

As a mechanical "buffer" for cells, keratin poses an interesting system to study from a biophysical perspective. Questions that manifest themselves in this context relate, for example, to the underlying mechanisms or material properties by which keratin structures are able to provide the cells with resilience to external forces. To address these points two sets of experiments are conducted, which are presented and discussed in detail in this thesis. In one set of experiments, we analyze the influence 
of shear forces on the active behavior of keratin networks in living cells. To this end, microfluidic methods are used to be able to expose the cells to well-controlled shear stress. The induced cellular response is extracted by observing and analyzing the motion of the keratin network. Our results shed light on a short-time active reaction of cells to external, mechanical influences and has consequences for the role of cytoskeletal cross-talk for cellular mechanics. In a second experimental block, we investigate the inherent, mechanical properties of keratin in living cells by analyzing buckling events in cellular networks. Here, our focus on keratin bundles in live cells, for which we also adapt a theoretical model to take the nature of the bundle into account, helps to further our understanding of the bundling process in vivo and gives new insights about the strength of inter-bundle linkage.

The thesis is organized in the following way: At first, an overview of the relevant scientific background is given (chapter 2). Here, keratin is introduced as part of the cytoskeleton and previous results regarding the influence of shear forces on keratin structures in cells are presented. Furthermore, the nature of buckling events are described for various types of filaments.

In chapter 3, details are given about the experiments that were carried out. This chapter contains information about the cell culture, the fabrication of microfluidic devices, the experimental procedure that was followed, and furthermore describes the principle of (confocal) fluorescence microscopy.

Chapter 4 comprises the applied methods and results of the shear stress experiments. It begins with a description of microfluidic flow in general and also specifies the velocity and shear stress profiles within our microfluidic devices. After describing image processing procedures, the chapter continues by outlining the methods employed for data analysis. Finally, our results of the influence of shear stress on the motion of keratin networks in live cells are presented and discussed.

Naturally occurring buckling events of keratin bundles in live cells are the topic of chapter 5. Here, we first describe the theory behind buckling events which we extend to match buckling of bundles that are formed from multiple individual filaments, which may slide against each other. Afterwards, the procedure for the evaluation of buckling events is explained. Finally, we present the obtained results followed by a subsequent discussion. In this context, we also address buckling events under shear stress conditions and debate implications for the influence of shear stress on cells.

Chapter 6 finally summarizes the work by presenting the main results and the conclusions derived. 


\section{State of the Art}

In the following, the biological and biophysical background of this thesis and ongoing investigations are discussed. After a short introduction to the cytoskeleton and in particular intermediate filaments (IFs), an overview of current opinions regarding the IF keratin is given. Furthermore, results from previous studies about the effect of shear stress on cells are described in addition to a depiction of current research on buckling events in cytoskeletal filaments.

\subsection{Cytoskeleton}

The cytoskeleton is a protein network distributed over the whole cytoplasm. It is a dynamic and adaptive structure which is continuously undergoing changes in morphology and network motion. In eukaryotic cells, the cytoskeleton consists of three types of cytoskeletal filaments, which are microtubules (MTs), micro- or actin filaments (MFs) and intermediate filaments (IFs), in combination with various associated proteins [1]. It fulfills a variety of functions starting with the definition of the cell shape and the organization of cell components over intracellular transport up to force generation, implying cell motility, and the protection of the cell against external influences [1,2].

IFs receive their name from the fact that their 10-nanometer diameter lies inbetween that of MFs (about $8 \mathrm{~nm}$ ) and MTs (about $25 \mathrm{~nm}$ ) [1]. Since MTs and MFs are assembled from polar globular proteins (G-actin and $\alpha$-, $\beta$-tubulin, respectively), they have a polar overall structure which enables directed motor protein activity. By contrast, IFs exhibit no polarity. The IF proteins, which are encoded by at least 70 genes [3], are subdivided into six classes based on their assembly properties, amino-acid-sequence and tissue association (overview given in table 2.1) [4,5]. All IFs are formed of building blocks with a tripartite structure consisting of an $\alpha$-helical 


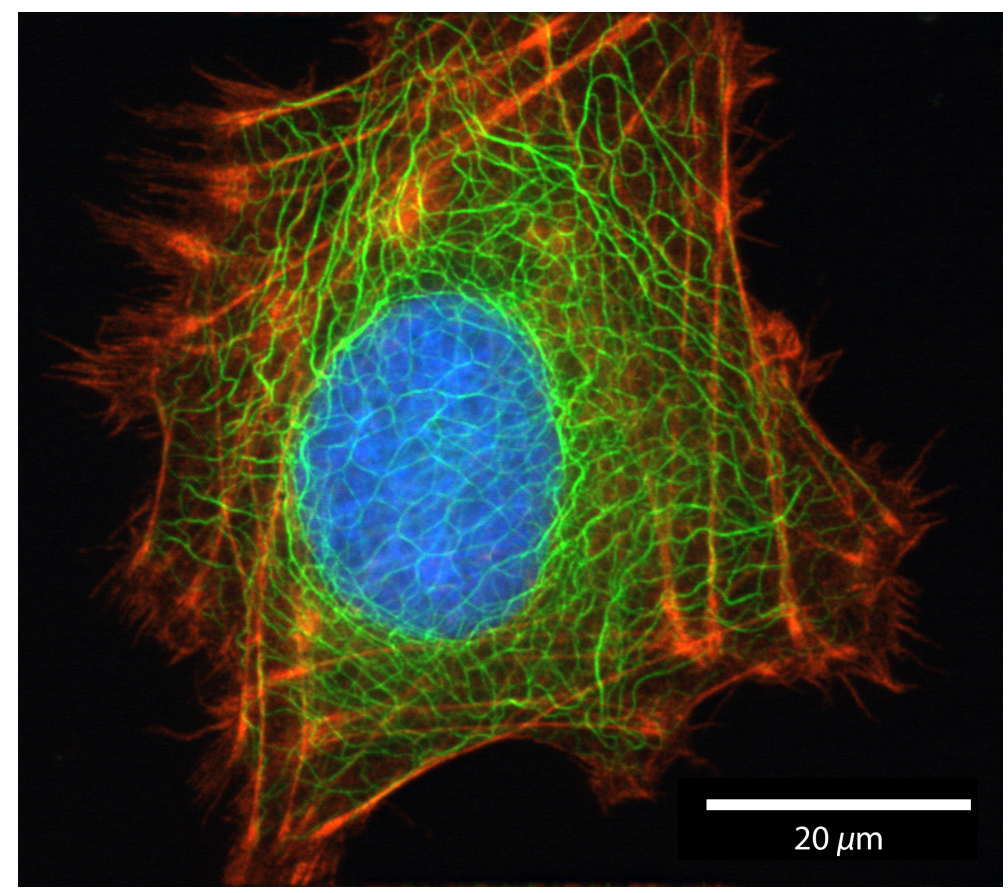

Figure 2.1: Fluorescence microscopy image of a SK8/18-2 cell showing parts of the cytoskeleton: the keratin intermediate filaments are depicted in green, the actin network in red and the DNA in blue. Image provided by courtesy of Jannick Langfahl-Klabes.

rod domain, which is flanked by an N-terminal (head) and a C-terminal (tail) (see also figure 2.2. While for vertebrates the rod domain of IFs is highly conserved [6], the head and tail domains vary distinctly in length and sequence throughout the IF protein family [6,7].

\subsection{Keratin - an Intermediate Filament Protein}

As depicted in table 2.1, keratins comprise class I and II of the IF protein family. Keratins are the largest and most heterogeneous group of cytoplasmic IF proteins. The majority of genes related to IF proteins in the human genome (at least 55 genes) encodes keratin proteins [8]. These proteins are divided into three categories: epithelial keratins, hair keratins and keratin pseudogenes. In the nomenclature, human keratins are additionally distinguished from non-human keratins. Each keratin is furthermore attributed a number for distinction. An overview of the numbering in each keratin category is shown in table 2.2 .

The category of hair keratins also includes other 'hard'-keratins (e.g. keratins from wool, nail, hooves or feathers), which are some of the most important structural proteins in vertebrates 9 . However, most genes encode for keratin in epithelial cells, where the keratin constitutes the major structural protein as well [10]. The epithelial 


\begin{tabular}{lll}
\hline type & IF protein & localization \\
\hline I & acidic keratins & epithelia, hair, horn, etc. \\
II & basic-neutral keratins & epithelia, hair, horn, etc. \\
III & vimentin & mesenchymal cells \\
& desmin & muscle cells \\
& glial fibrillary acidic protein & astrocytes \\
& synemin & muscle cells \\
& peripherin & diverse neuronal cells \\
IV & neurofilaments & neurons \\
& nestin & neuroepithelial stem cells \\
& $\alpha$-internexin & neurons \\
V & lamins & nucleus \\
VI & filensin & lens cells \\
& phakinin & lens cells \\
\hline
\end{tabular}

Table 2.1: Overview of the six classes of intermediate filaments [4,5].

tissue can be categorized into simple (single-layered) and complex (multi-layered) types. In general, the type of keratin which is expressed in any cell is highly specific to the cell type [11, 12]. Keratin K8 and K18 are, for example, the most prominent keratins expressed in simple epithelial tissue like liver tissue.

As mentioned before, the assembly of IFs differs from those of MTs and MFs since the IF subunits assemble in a hierarchical fashion. Within the IF family, the keratins (assembly group I: type I and II of IF classification) further distinguish themselves in their assembly properties from other IF proteins such as vimentinlike proteins (assembly group II: type III and IV of IF classification) and lamins (assembly group III: type V of IF classification) [7]. Uniquely for keratins, one

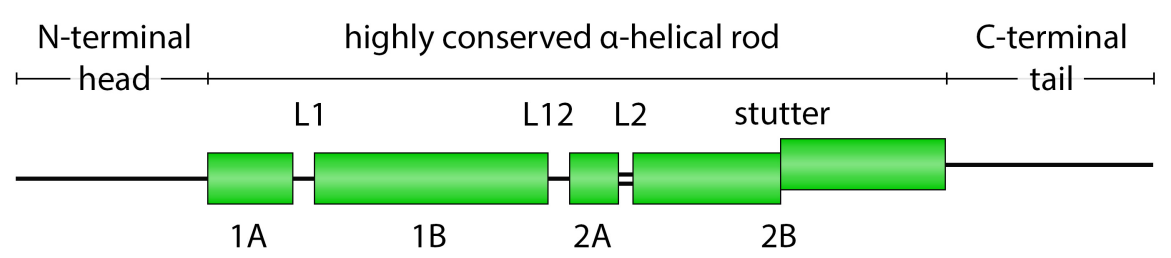

Figure 2.2: All IF proteins share the same characteristic tripartite structure: An $\alpha$ helical rod domain is flanked by an $\mathrm{N}$ - and a C-terminal. The rod is subdivided into four $\alpha$-helical segments (1A, 1B and 2A, 2B) interrupted by short linker sequences (L1, L12, L2). For all IF proteins from vertebrates, excepting lamins, the rod domain is highly conserved with regard to structure and length. Image adapted from reference [4]. 


\begin{tabular}{ll}
\hline category & number range \\
\hline human type I epithelial keratins & $9-28$ \\
human type I hair keratins & $31-40$ \\
non-human type I epithelial and hair keratins & $41-70$ \\
human type II epithelial keratins & $1-8$ and 71-80 \\
human type II hair keratins & $81-86$ \\
non-human type II epithelial and hair keratins & $87-120$ \\
type II keratin pseudogenes & $121-220$ \\
type I keratin pseudogenes & $221-?$ \\
\hline
\end{tabular}

Table 2.2: Overview of the numbering scheme in the keratin categories [8. Note that the type I keratin pseudogenes, as the last category, have an open system starting with number 221.

acidic monomer keratin of type I and one basic-to-neutral monomer keratin of type II build a coiled-coil heterodimer. By contrast, vimentin-like proteins form homodimers consisting of two building-blocks from the same class of proteins. For keratins, two heterodimers then align in an anti-parallel and partly overlapping way to build tetramers, which assemble laterally to so-called unit-length-filaments (ULFs) comprising four tetramers [7,13,14]. The ULFs finally build the $10 \mathrm{~nm}$ thick keratin intermediate filaments (KIF) by annealing longitudinally.

Another aspect in which keratin assembly differs from vimentin-like proteins lies in the fact that keratin ULFs are composed of only four tetramers (in place of typically eight tetramers for vimentin-like proteins). Interestingly, the decrease in the number of tetramers within keratin ULFs has no influence on the final filament diameter, which is about the same for vimentin and keratin. It has been suggested that this fact is caused by the lack of a compaction step for keratin which, in contrast, occurs for vimentin-like proteins during the filament elongation phase [14]. Another feature particular to keratins is the assembly speed which is about 80 times faster than, for example, for vimentin. Accordingly, the ULF-building phase has only been observed in vitro for low protein concentrations [14]. Since the buffer systems are not identical for the assembly of keratin and vimentin, however, the comparison between the two assembly speeds is debatable.

\subsubsection{Aggregation of Keratin Filaments}

The assembled keratin filaments can be treated as polyelectrolytes, meaning that they are polymers whose subunits are charged (e.g. K8K18 dimers have a net charge 
of -17e [15]). Charge-related electrostatic interactions between polyelectrolytes are described by polyelectrolyte theory. Since many intracellular biopolymers, including DNA, actin, microtubules and intermediate filaments, are characterized by large negative net charges of their monomeric constituents, their in vitro behavior is often governed by similar principles which can be explained by polyelectrolyte theory 16. In the following, in vitro structure formation is discussed in particular. However, electrostatic interactions are not only relevant in vitro, they also play a role in vivo, e.g. during targeting of actin cross-linking proteins or motor proteins to actin filaments.

As reviewed by Janmey et al., polyelectrolytes establish links between each other when counterions are present 16. Here, the counterions serve a double purpose 17. First, they reduce the repulsion between the polyelectrolytes by shielding their charges. Secondly, they provide an attractive interaction between polymers which results in polymer-counterion-polymer contact points. This can lead to a number of structural morphologies depending on e.g. polymer- and ion-concentration or polymer characteristics such as length, stiffness and charge distribution on the filament surface [16]. Prominent among the achievable effects are the assembly of networks or the emergence of bundles. Interestingly, bundle formation occurs only for sufficiently high counterion concentrations. The critical ion concentration for bundle formation, in turn, depends on ion valence but is hardly affected by ion structure or the type of polyelectrolyte. Thus, similar critical ion concentrations ( $\gtrsim 10 \mathrm{mM})$ have been identified for $\mathrm{Ca}^{2+}$ - and $\mathrm{Mg}^{2+}$-induced bundle formation in F-actin 17 and vimentin 18 . Furthermore, the structurally different cations spermine $\mathrm{Sp}^{4+}$ and the antimicrobial agent $\mathrm{CSA}_{1} 3^{4+}$ both initiate bundling in F-actin and DNA at sub mM concentrations (reviewed in [16]).

For keratins, in vitro experiments confirm that the filament elongation as well as network and bundle formation is regulated by concentrations of at least monoor divalent salts 1921 . Bundle formation is mediated, in particular, by $\mathrm{Mg}^{2+}$ concentration above $\sim 1 \mathrm{mM}[22]$. Furthermore, it is currently under discussion whether the C-terminal of the keratins plays a key role in mediating the crosslinking interactions during bundling. The idea is suggested in analogy to findings for neurofilaments, for which the C-terminal was revealed to protrude from the filament 23,24]. Supporting the idea, Lee and Coulombe have found evidence for the involvement of the C-terminal for K5K14 bundle formation 20]. In addition, they have shown that the head-1A domain and the L2-2B regions play similarly important roles (compare with figure 2.2). It should be noted, however, that although these studies make an involvement of the C-terminal for K8K18 assembly plausible, they 
do not provide conclusive evidence. Thus, it is debatable whether the C-terminal protrudes from keratin filaments in the way it does for neurofilaments, since the former have distinctly shorter tail domains as compared to the latter. Likewise, it is yet unknown whether the findings for K5K14 keratins are also valid for K8K18 keratins, which we are interested in.

The organization of keratin in the cell is further mediated by proteins (see e.g. review 25]). On the one hand, some proteins have been identified which are directly involved in the bundling process of individual keratin filaments, namely filaggrin [26] and filaggrin-2 [27]. Other compounds, such as trichohyalin [28], repetin [29] and hornerin [30], are also considered to play a role in keratin bundling, although their function is still being discussed. On the other hand, proteins are also involved in the regulation of the keratin network and the keratin bundles. As evidenced by the examples described in the following, this implies not only keratin-keratin cross-links but also the interactions between keratin and other constituents of the cytoskeleton.

Plectin is a known linker between various filaments of the cytoskeleton and also plays an important role in keratin linkage 31,32 . In this context, OsmanagicMyers et al. have shown that in plectin-deficient keratinocytes the bundle thickness is enhanced, probably due to a collapse of finer bundles [33]. By contrast, they have observed orthogonal cross-bridges in between numerous thin bundles stabilizing the network in wild-type cells. From the two observations they conclude that plectin is involved in the formation of IF cross-links. Also, other members of the plakin protein family are similarly related to keratin [34 and play a role in KIF reorganization. Epiplakin is one example and has been shown to associate with keratin when stress (e.g. osmotic shock or UV irradiation) is applied to cells. As a result, the keratin network is more stable. Evidence to the fact is provided by experiments on epiplakin deficient cells demonstrating that the KIF network disruption occurs faster for this type of cell 35.

Another factor which influences keratin properties is phosphorylation. It is important for the assembly state of keratin and thus the KIF bundling and network organization, as well as the solubility and conformation of the protein (see review 36] for details). Phosphorylation takes place at multiple sites preferentially within the C- or N-terminal and is regulated by several kinases. It is also involved in the reaction of cells to external disturbances like mechanical or chemical stresses 36 38 (see also section 2.2.4). 


\subsubsection{Mechanical Properties of Keratin}

In vitro experiments using rheology have revealed that IF networks show a viscoelastic behavior with strain hardening effects [39,40]. These effects are explained by Storm et al. as a response counteracting the decrease in entropy caused by the pulling out of fluctuations in the filaments' shape during filament stretching [41] (see also section 4.5. In general, strain hardening is, however, primarily observed when networks are cross-linked [40].

Ma et al. have revealed using rheology measurements that keratins also demonstrate strain hardening. Moreover, they have observed that uniquely for keratins a repeated imposition of small deformations leads to a progressively stiffer protein suspension [42]. Remarkably, both keratin and vimentin are able to quickly recover their original state after shear forces cease to be applied. In a separate study, Ma et al. have compared the in vitro mechanical properties of a wildtype keratin network and the network of a K14 mutation which is characterized by inhibited ion-induced bundle formation [19]. Based on rheology data, they have found that bundling in keratin networks increases the resilience of the network against large deformations.

Further mechanical properties in form of filament extensibilities have been studied for keratin by Kreplak et al. By laterally deforming single keratin filaments with an AFM tip, they have shown in in vitro experiments that keratin filaments are highly extensible and can be stretched by a factor of more than 2.5 [43. Fudge et al. have later revealed that keratinocytes which are subject to uniaxial cell strains of $133 \%$ can not only survive these deformations but show only minor damage in their KIF network [44].

\subsubsection{Keratin in Cells}

The mechanical properties of IFs, such as their nonlinear strain hardening behavior, provides evidence that IFs serve as a mechanical buffer system which helps the cell withstand potentially harmful external mechanical influences [38, 45, 46]. As shown in in vitro experiments, keratin filaments also tend to form bundles which enhance the mechanical stability of the cell in vivo [20,47]. Indeed, Ramms et al. have revealed by analyzing the mechanical properties of keratinocytes with atomic force microscopy (AFM) and magnetic tweezers that keratin-deficient cells are much softer than wildtype cells and hence they have demonstrated the importance of the contribution of keratin filaments to the mechanical properties of cells [48]. Furthermore, Seltmann et al. have shown by performing microfluidic optical stretcher experiments that keratin-free cells exhibit a deformability which is $60 \%$ higher in 

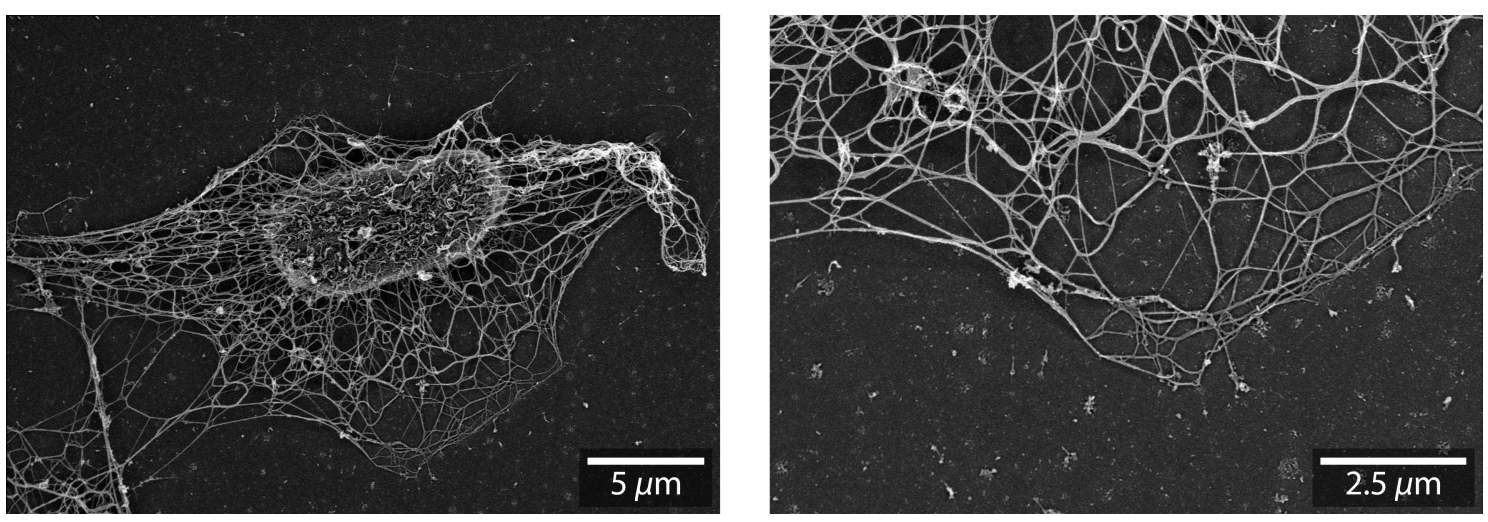

Figure 2.3: SEM images of a keratin network which has been extracted from a cell. Images provided by courtesy of Prof. Dr. P. Walter and Prof. Dr. M. Beil, University of Ulm.

comparison to wildtype cells 49 .

The keratin network inside cells, as it is shown in a fluorescence image in figure 2.1 and in a scanning electron microscopy (SEM) image in figure 2.3, cannot be understood as a static structure, such as the bone-skeleton inside a body, since it is a very dynamic network with ongoing restructuring. The KIF bundles furthermore show distinct oscillations [50] and a propagation of waveforms along their long axis 51. Additionally, the whole network undergoes a continuous assembly and disassembly cycle [52] (see figure 2.4): The assembly of keratin particles occurs at the cell periphery from a non-filamentous pool of keratin proteins in the cytoplasm close to focal adhesions. While these particles elongate by continuous end-to-end annealing of further KIF subunits, they move preferentially along actin fibers and occasionally also along microtubules 53 towards the nucleus. During their translocation to the nucleus they are integrated into the existing network and form bundles. Finally, KIF particles are dissolved again near the nucleus and diffuse into the cytoplasm, or form a stable perinuclear cage [54].

IFs are also discussed with regard to their importance to mechanical signal transduction since the filaments are associated with components of cell-to-cell and cellto-substrate adhesion sites [55]. Keratin, in particular, is linked with both integrinand cadherin-associated cell surface junctions like hemidesmosomes and desmosomes [56, 57]. Furthermore, it is associated with the outer nuclear membrane via plectin-nesprin-3 cross-links 58 .

Further functions of keratin, which are only briefly described in the following, are related to cellular growth, motility and apoptosis [59]. Studies have revealed, for example, the involvement of keratins in embryonic development, where keratin deficiency in embryonic epithelia leads to malplaced desmosomes and fatal growth 


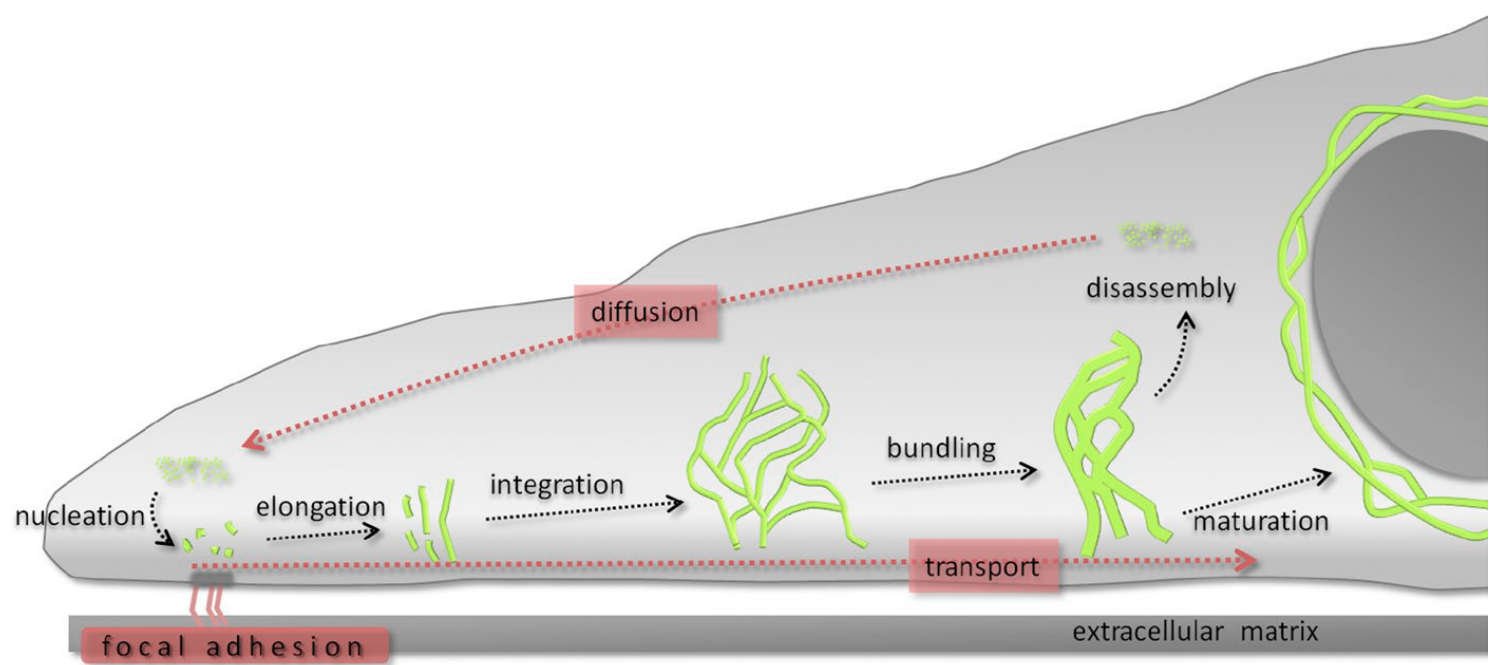

Figure 2.4: The keratin network inside cells follows a continuous assembly and disassembly cycle. The assembly process starts near focal adhesions where keratin particles are formed. While moving along actin fibers towards the nucleus, they are further elongated by end-to-end annealing. The resulting filaments are integrated into the existing network and form bundles. Finally, they disassemble again or build a stable cage-like structure around the nucleus. Image taken from reference [54].

retardation [60]. Cells which have been treated with sphingosylphosphorylcholine (a bioactive lipid which is present in ovarian cancer patients) show a decrease in the average elastic modulus of the cell, originating from perinuclear collapse of the keratin filaments [61]. In consequence, the cells are able to deform more easily and move through smaller gaps, thus enhancing overall cell mobility.

With the functions of keratin being of structural support and regulation of metabolic processes, there is also a broad spectrum of skin, oral, hair and liver diseases which are connected to keratin malfunction [59,62,65]. An example of a skin fragility disorder is the skin blistering disease, epidermolysis bullosa simplex, caused by mutations in K5 and K14 keratins, which lead to a loss of structural integrity under external mechanical stress [66]. Malfunctions of the keratins K8 and K18 are known to cause a predisposition for chronic [67] and acute 68] liver diseases. Since keratin pairing is specific to the cell type, keratins are often used as markers for cancer diagnostics (overview given in [64]). Apart from these diagnostic features, keratin's role during cancer cell invasion and metastasis is also under current scientific discussion 64]. 


\subsubsection{The Role of Keratin in the Cellular Response to Stress}

Keratin has been revealed to play a key role in the cellular response to various types of stress (e.g. UV, oxidative or chemical stress; see reviews [11, 59]), in particular to mechanical forces, as discussed in the following. Upon application of shear stress, several properties of keratin alter, leading to structural changes in the network which depend on the shear stress exposure time. For shear stress of $3 \mathrm{~Pa}$ applied to cells over $24 \mathrm{~h}$, the KIF network deteriorates resulting in disorganization and a large amount of free keratin particles and squiggles 69,70. For shorter time periods of shear stress exposure (1.5 Pa over $4 \mathrm{~h})$, Sivaramakrishnan et al. have revealed a reorganization of the network. They have found that the KIF network mesh sizes decrease near the cell periphery, which results in a more uniform mesh size distribution across the entire cell. As a consequence, the mean storage modulus of the cell increases by $40 \%$, according to measurements using particle-tracking microrheology [71. For even shorter periods in time, where a network reorganization is not observable yet, the properties of the keratin bundles themselves already change. After 1-4h of shear stress, the formation of tonofibrils, which are comprised of KIF bundles, have been observed by transmission electron microscopy (TEM) images 72 . The remodeling of the KIF network is then maintained at least for $24 \mathrm{~h}$. Sivaramakrishnan et al. have further investigated the subunit exchange of KIF bundles in shear-stress-exposed cells using fluorescence recovery after photobleaching (FRAP). They have found that the exchange rate is about doubled under the influence of shear stress (FRAP recovery time: $t_{1 / 2}=55 \pm 16$ min versus $t_{1 / 2}=120 \pm 22 \mathrm{~min}$ without shear stress) $[72]$. Flitney et al. have also reported that the soluble pool of free keratin particles is distinctly reduced when cells are exposed to shear stress ( 0.7 or $1.5 \mathrm{~Pa}$ for up to $10 \mathrm{~h}$ ) [38]. These particles are hence thought to attach to preexisting KIF bundles and to be eventually integrated into the keratin network.

Important for the reorganization of the keratin network is an increase in the phosphorylation of keratin for both keratin types which make up the filaments and particles. Using immunofluorescence, Flitney et al. have identified in particular two amino acids (serine residues 73 and 33, respectively) which are phosphorylated in K8K18 as a response to shear stress. This, in turn, enables more keratin particles to be integrated into the preexisting keratin network [38]. Sivaramakrishnan et al. have further revealed that the phosphorylation is mediated by the protein kinase $\mathrm{C} \zeta(\mathrm{PKC} \zeta)$, at least in the case of serine 33 on K18. Furthermore, they have shown the importance of the shear stress induced KIF network reorganization in cells: While the inhibition of PKC $\zeta$ has no effects on cells in the absence of shear 
stress, it results in a crucially reduced cellular viability in cells which have been exposed to external shear forces 38.

In sum, a soluble pool of keratin is believed to make up a reservoir which can be recruited by the cells at need. By reinforcing the preexisting network, the additional keratin helps the cells to withstand shear forces more efficiently. The reorganization due to shear stress is mediated by phosphorylation of specific amino acids in the keratin, a process in which protein kinases are involved.

Altogether, the study of keratin under cellular shear stress conditions is most important in view of the function of keratin as a cellular buffer system, a role that is also highlighted by the diseases associated with keratin malfunction and mutations. Previous investigations on the structural reorganization of keratin have shed light on the long-term response of cells to external mechanical influences. However, not much is known about the intermediate steps that cells take on time scales of minutes to protect themselves against external forces. To bridge the gap, we analyze the keratin network within minutes after shear stress application. Furthermore, we focus on the change in KIF bundle motion to conclude upon a possible short-termed alteration in the mechanical properties of the cellular environment. A description of the experimental work involved as well as the results obtained is provided in chapters 3 and 4 .

\subsection{Buckling Events in Cells}

An elastic rod to which a sufficiently high compressive force is applied at its ends buckles along its full length (Euler Buckling). This behavior is also present in cells, where rod-like parts of the cytoskeleton are compressed and deflected accordingly. However, the buckling characteristics are changed since the filaments are surrounded by the cytoplasm, which is comprised of other constituents of the cytoskeleton, organelles and the cytosol. In such a case of constrained buckling, the buckling wavelength is shorter and the deflection arc spans only part of the filament length (see figure 2.5). Although the curvature is high for short wavelengths, this sort of buckling ultimately consumes less energy since the cytoplasm is deformed less.

In their paper, Brangwynne et al. have described experiments on buckling events of microtubules in cells as a result of both naturally occurring intracellular and exogenous compressive forces 73]. Thus, in one set of experiments they have observed buckling induced by polymerization of MTs against the cell cortex at the cell periphery. As also observed previously $[74 \sqrt{76}]$, they have found that the generated forces lead to a multi-peak, short-wavelength deflection of the MTs. Brangwynne 


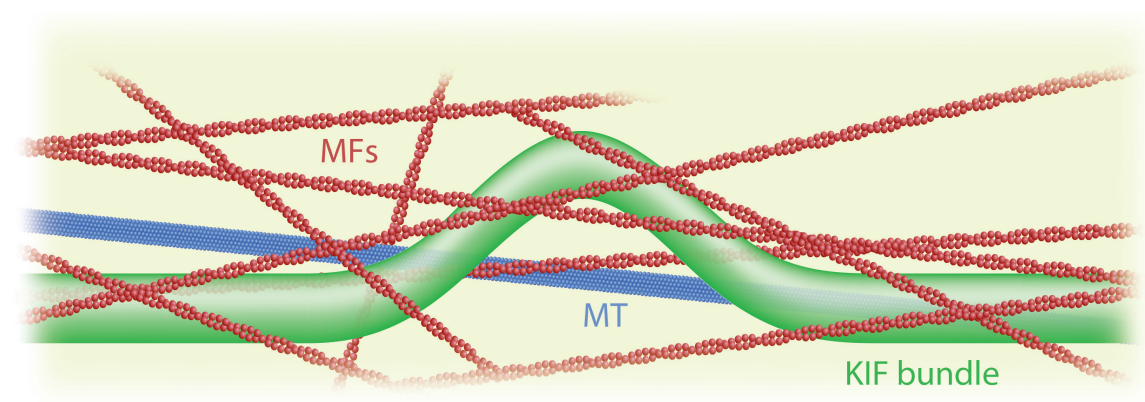

Figure 2.5: Due to compression forces, filamentous members of the cytoskeleton show buckling events. The resulting buckling wavelengths are not only defined by the intrinsic properties of the rod but are also a result of the mechanical attributes of the surrounding matrix (i.a. other constituents of the cytoskeleton).

et al. have moreover revealed the wavelength of buckling due to polymerization to be of the same length $(\sim 3 \mu \mathrm{m})$ as for buckling which has been induced in vivo in MTs by applying an external compressive force using a micromanipulator. Additionally, myosin driven actin contraction has been found to cause buckling events of MTs both inside cells and in in vitro experiments 73,77$]$. Actin, however, has yet another effect on buckling. In fact, the MFs influence the buckling events by their mere presence. As they surround the MTs they contribute to the stiffness of the cytoplasm, which affects the buckling wavelength. Evidence to the purpose is once more provided by Brangwynne et al., who have found a buckling wavelength of about $4 \mu \mathrm{m}$ instead of approximately $3 \mu \mathrm{m}$ for cells which were treated with cytochalasin - a drug which disrupts the actin network 73.

Apart from corresponding observations in MTs, buckling events have also been seen for actin stress fibers 78 and keratin [44]. For example, Lu et al. have measured the waveforms generated in actin stress fibers by a shortening of the pre-stretched flexible membranes on which the cells were cultured [78. The buckling wavelength was determined to be about $2 \mu \mathrm{m}$. According to the theory briefly mentioned above, they have furthermore revealed that the wavelength increases when the influence of the surrounding matrix is decreased by disrupting MTs or IFs through the addition of nocodazole or acrylamide, respectively.

Fudge et al. have also observed buckling of keratin after first uniaxially stretching cells up to a final length that is $233 \%$ of their original size and then allowing the cells to relax [44]. They have determined the wavelength of keratin bundles on TEM images to be about $1.7 \mu \mathrm{m}$ during cell relaxation. To be able to apply a theoretical model to their buckling data, they have furthermore characterized the keratin bundles. They have thus found the average number of individual filaments 
in a longitudinal cut to be about seven according to the images. Based on this parameter and assuming hexagonal packaging of the $10 \mathrm{~nm}$ thick filaments within a bundle, they have estimated the bundle diameter to be on average $64 \mathrm{~nm}$. Using the average buckling wavelength and the estimated average bundle diameter Fudge and colleagues have applied a theoretical model established by Brangwynne et al. [73] to conclude upon a stiffness of the surrounding matrix of about $1 \mathrm{kPa}$.

The model of Brangwynne et al. which was used, for example, by Fudge et al. is briefly presented in the following together with suggestions for further extensions that have been proposed. An in-detail derivation of the theory is given in chapter 5.1. The model considers the following equation for the energy of constrained buckling 73 :

$$
E=\frac{\kappa}{2} \int \mathrm{d} x\left(u^{\prime \prime}\right)^{2}-\frac{f}{2} \int \mathrm{d} x\left(u^{\prime}\right)^{2}+\frac{\alpha}{2} \int \mathrm{d} x(u)^{2} .
$$

Here, $u$ represents the transverse displacement of the rod as a function of the axial coordinate $x$. The first term in equation 2.1) describes the bending energy of the $\operatorname{rod}(\kappa$ is the bending rigidity). The second term represents the axial compression energy released by the buckling (with $f$ as the compressive force acting on the rod). Finally, the third term expresses the energy which is needed for the linear elastic deformation of the surrounding medium when the rod pushes through it while bending ( $\alpha$ is a measure for the elastic modulus of the medium). The reduced wavelength (in comparison to Euler buckling) is hereby expressed by $\lambda=2 \pi\left(\frac{\kappa}{\alpha}\right)^{1 / 4}$. The model has been further adapted by Das et al. and Shan et al. The first group of authors presents the more complete extension which additionally allows for nonlinearities in the coupling between rod and medium, and takes longitudinal deformations of the surrounding matrix into account 79. By contrast, Shan et al. include only the extra energy term relating to the longitudinal deformation of the surrounding medium [80. Following a numerical ansatz, Das et al. have found that both the threshold force for buckling $f_{\text {crit }}$ and the buckling wavelength $\lambda$ remain approximately the same for the nonlinear and linear system [79. Shan et al. further state that these two parameters are also independent of the longitudinal deformation in the medium. Hence, the critical force for buckling $f_{\text {crit }}$ as well as the buckling wavelength $\lambda$ remain unaffected by the two extensions of the original model by Brangwynne et al. The extra energy terms, however, affect the amplitude of buckling. In fact, they lead to buckling with multiple wave peaks, where the amplitude follows an exponential decay. Thus, both Das et al. and Shan et al. express their solutions by the following equation 


$$
u(x)=u_{0} \exp (-x / l) \sin (q x),
$$

where $l$ represents the decay length and $q$ is connected to the buckling wavelength by $\lambda=2 \pi q$. According to Shan et al., the decay length $l$ is dependent on the bending rigidity $\kappa$ of the rod and a parameter $\alpha_{\|}$which relates both to the elasticity of the surrounding matrix and the strength of longitudinal coupling between rod and matrix $\left(\right.$ i.e. $\left.l \sim\left(\kappa / \alpha_{\|}\right)^{1 / 4}\right)$. However, for strongly non-linear coupling between rod and matrix there is a transition to a regime, where $l \sim\left(1 / \alpha_{\|}\right)^{1 / 2}$ or more precisely $l \sim\left(\beta / \alpha_{\|}\right)^{1 / 2}$ with the nonlinear elastic constant $\beta[79,80$. Depending on the exact ratio between $\kappa$ and $\alpha_{\|}$or $\beta$ and $\alpha_{\|}$the decay length is shorter or longer, which ultimately also defines the number of wave peaks visible in the buckling.

Further studies follow different approaches for a theoretical description of constrained buckling, e.g. treating the rod environment as viscous, elastic or viscoelastic [81]. In another publication, Li et al. have set up a model similar to Brangwynne et al. with a small modification consisting of a surrounding matrix that is comprised of two individual components: a filamentous network and a fluid cytosol 82 . While the network delivers an elastic term to their equation of motion, the cytosol is accounted for by including viscous contributions to their model. In the two studies 81] and [82], the authors conclude that the conformation of the buckling wavelength is solely defined by elastic properties (yielding similar expressions for $\lambda$ to the one from Brangwynne et al.), while the viscosity of the surrounding matrix determines the dynamics of buckling i.e. the growth rate.

Although the theory of constrained buckling for a single elastic rod has received much attention, little is known about how buckling is changed if several rods (i.e. filaments) are jointly bent within a bundle that buckles. In our study, we address this point by extending the theory from Brangwynne et al. To this end, we introduce a coupling parameter which describes the ability of individual filaments within a bundle to slide against each other. Furthermore, we apply the adapted theory to naturally occurring buckling events of keratin bundles in live cells to conclude upon the strength of inter-linkage in a bundle. The corresponding experiments and our findings are described in chapters 3 and 5 . 


\section{Materials and Methods}

Our experimental work was performed on a cellular model system of SW-13 cells, into which fluorescent keratins were introduced via stable transfection of the cells. Details about the cell line as well as cell culture steps are provided in section 3.1 at the beginning of this chapter. Subsequent experiments on the cells are composed of two parts. Both sets of experiments employed microscopy techniques, the principles of which are reviewed in section 3.2 . In the first set of experiments, the cells were exposed to microfluidic flow and the cellular response to the flow-induced shear was analyzed by monitoring changes in the keratin network dynamics using fluorescence microscopy. The protocol followed for these experiments is explained in section 3.3 . while the fabrication of microfluidic devices is specified in section 3.4. To estimate the shear stress distribution across a cell in a microfluidic channel, we have furthermore carried out finite element method (FEM) simulations, which are described in section 3.5. The second set of experiments is focused on the mechanical properties of both the cellular keratin network as well as its surrounding matrix. To that end, we have studied buckling events of keratin bundles in cells under no-shear conditions using confocal microscopy. Particulars for this specific experimental block are represented in section 3.6 .

\subsection{Cell Culture}

Our cells were obtained from subclones of a human adrenal cortex carcinoma-derived cell line SW-13 (ATCC CCL-105) [83], which has been stably transfected with DNA encoding for fluorescent human keratin hybrids (HK8-CFP, HK18-YPF) [47, 53, 84]. The resulting SK8/18-2 cells thus express a network of KIFs that is observable with fluorescence microscopy techniques. Both the SW-13 and the final SK8/18-2 cell line were kindly provided by Rudolf Leube (RWTH Aachen, Germany). 
For cell culture, we used Dulbecco's Modified Eagle's medium (DMEM) with a high glucose concentration of $4.5 \mathrm{~g} / \mathrm{l}$ (PAA E15-810, PAA Laboratories GmbH, Pasching, Austria) mixed with $10 \%$ fetal calf serum (FCS, Invitrogen, Darmstadt, Germany), $100 \mathrm{U} / \mathrm{ml}$ penicillin and $0.1 \mathrm{mg} / \mathrm{ml}$ streptomycin (Pen- Strep; SigmaAldrich, München, Germany). The cells were maintained in a water vapor saturated atmosphere with $5 \% \mathrm{CO}_{2}$ at $37^{\circ} \mathrm{C}$ and passaged two times per week. To this end, the growth medium was removed from the cell culture dish and the cells were washed with a PBS/EDTA-solution (0.02\% (w/v) EDTA (Roth, Karlsruhe, Germany)). Subsequently, we incubated the cells with a trypsin/EDTA-solution $(0.25 \%(\mathrm{v} / \mathrm{v})$ trypsin (Sigma-Aldrich), 0.02\% (w/v) EDTA) until they began detaching from the ground surface. Afterwards, the trypsin/EDTA-solution was gently removed and the cells were completely detached by washing them with growth medium. The resulting cell-suspension was diluted as required and applied to a new cell culture dish.

\subsection{Microscopy Methods}

The investigation of living SK8/18-2 cells involved two microscopy methods: Epifluorescence and confocal microscopy. During all experiments, the cells were kept under physiological conditions by mounting an incubation chamber INUG2E-ONICS (Tokai Hit CO., Ltd, Gendoji-cho, Fujinomiya-shi Shizuoka-ken, Japan) on top of the microscope stage. Within this chamber, the atmosphere was saturated with water vapor containing $5 \% \mathrm{CO}_{2}$. Both the objective of the microscope and the chamber itself were warmed to $37^{\circ} \mathrm{C}$.

\subsubsection{Fluorescence Microscopy}

The ability of common brightfield microscopy techniques to resolve nm-sized features in a sample is limited according to Abbe's law by the wavelength of the light $\lambda$, the refractive index $n$ of the material in between lens and sample and the opening angle $2 \phi$ of the microscope objective: the resolution is given by $d=\frac{\lambda}{2 n \sin (\phi)}$ where $d$ describes the minimal distance between two objects which can still be distinguished from each other [85]. Fluorescence microscopy circumvents these limitations to a certain extent by the fact that the sample itself reemits light which can be then detected specifically. To this end, the sample has to contain fluorophores. The technique has two main advantages: First, the fluorophores can bind to and hence label specific parts of the sample that are of particular interest, e.g. keratin proteins. 
(a)

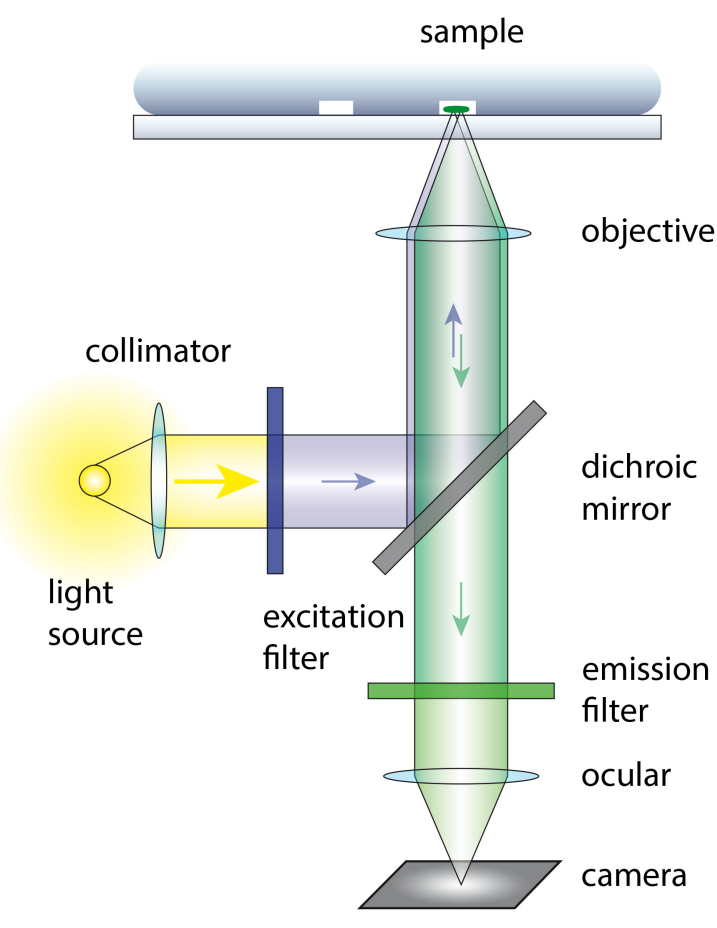

(b)

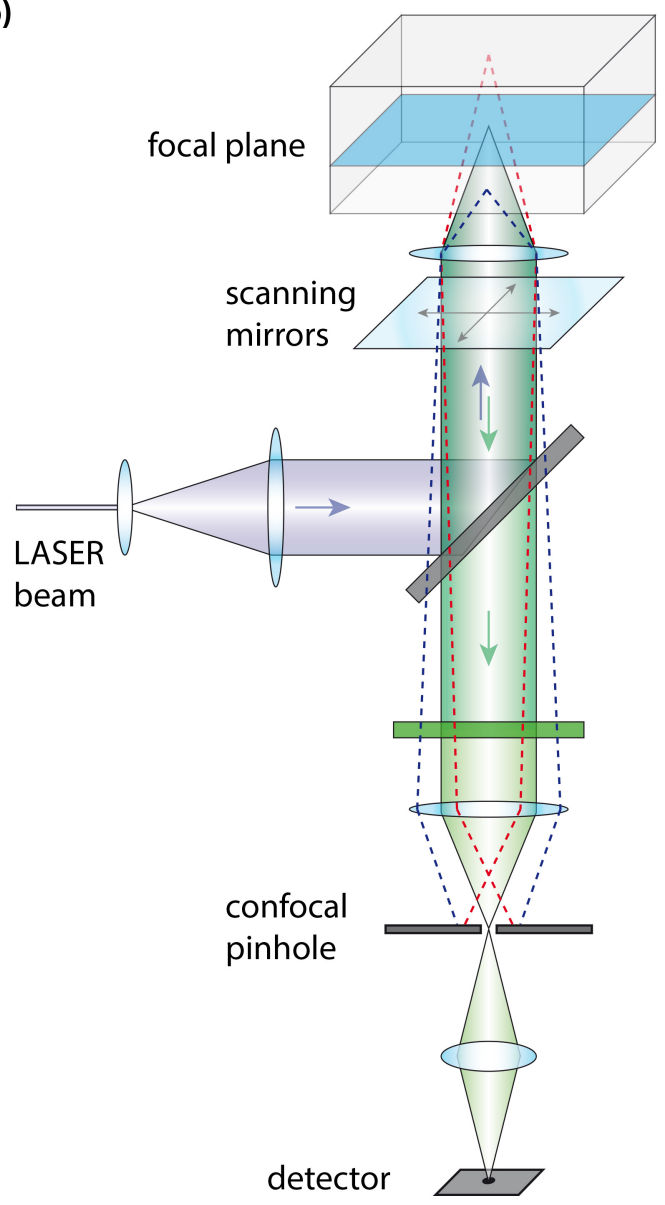

Figure 3.1: (a) Basic buildup of an inverted epifluorescence microscope with a common divergent light source and a single filter cube containing an excitation filter, an emission filter and a dichroic mirror. (b) Laser scanning confocal microscopes acquire the image pixel by pixel, a process which is controlled by scanning mirrors deflecting a laser beam. The confocal pinhole filters the signal depending on its origin in the sample, thus enhancing the in-depth resolution.

Secondly, the components to which the fluorophores are bound can be smaller than the wavelength of the incident light.

The microscope that we use for our experiments is an Olympus IX 81 inverted fluorescence microscope (Olympus Microscopy, Hamburg, Germany) with an Olympus MT20 illumination system including a $150 \mathrm{~W}$ Xenon arc lamp serving as light source. As depicted in figure 3.1 a , the light is collimated by a lens and filtered by the excitation filter. Here, the broad spectrum of the Xenon lamp is reduced to a small band so that only light with suitable wavelengths for the specimen is transmitted. Afterwards, the light is reflected by a dichroic mirror, which is only transparent for specific wavelengths (light which is emitted by the sample) and remains otherwise reflective. By illuminating the fluorophores with a certain wavelength, electrons are 


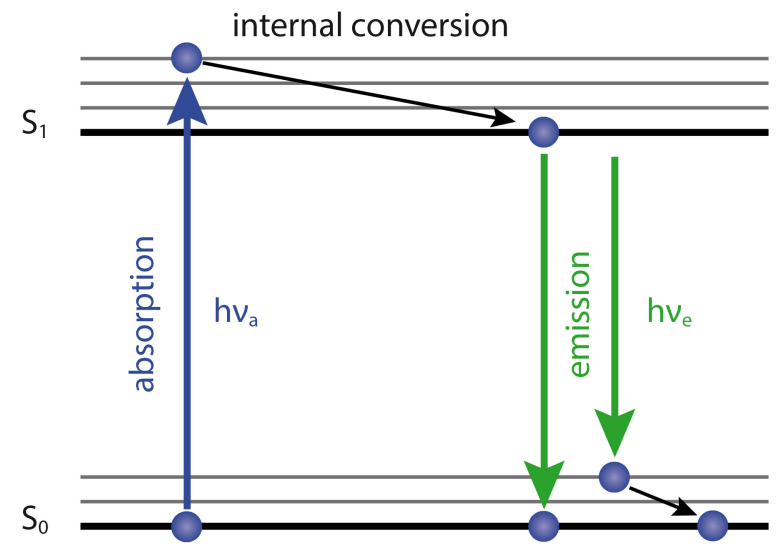

Figure 3.2: In fluorescence microscopy, electrons which are in an energetic ground state $\mathrm{S}_{0}$ are excited by absorbing external light. This excitation light with a suitable energy of $E_{\mathrm{a}}=h \nu_{\mathrm{a}}$ and a corresponding wavelength $\nu_{\mathrm{a}}$ elevates the electron to an higher energy level $\left(S_{1}\right)$. In each energy state, the electron can be in a different vibrational mode which is illustrated by gray lines. Usually, the electron is excited to a vibrational mode of higher energy but relaxes rapidly to the ground vibrational mode without the emission of light. The fluorescence takes place when the electron relaxes from the lowest excited energy state to a ground state under the emission of light with an energy $E_{\mathrm{e}}=h \nu_{\mathrm{e}}$.

elevated to an excited state. As the electrons subsequently relax to their ground state, they emit a photon with a wavelength longer than the excitation wavelength, since part of the energy has been dissipated as vibrations (process depicted in a so-called Jablonski diagram in figure 3.2 86.

This effect is called Stokes shift. The light emitted by the sample is then separated from the original excitation light by the dichroic mirror and the emission filter. In our setup, which was used for observing the SK8/18-2 cells, an excitation filter BP470-495, a dichroic mirror DM505 and an emission filter BA510-550 were combined in a U-MNIBA3 filter cube. The corresponding spectra characterizing these optical components are depicted in figure 3.3. After passing the filter cube, the light which was emitted from the sample is finally detected with a Hamamatsu Orca-R2 C10600-10B camera (Hamamatsu Photonics Deutschland GmbH, Herrsching am Ammersee, Germany) containing a CCD (resolution: $1344(\mathrm{H}) \times 1024(\mathrm{~V})$ pixels, with a pixel size of $6.45 \mu \mathrm{m} \times 6.45 \mu \mathrm{m})$, which is able to acquire images with 16 -bit gray color depth.

\subsubsection{Confocal Microscopy}

Laser scanning confocal microscopy enhances the resolution of epifluorescence microscopy by reducing the focal volume in a sample from which signal is acquired. As 


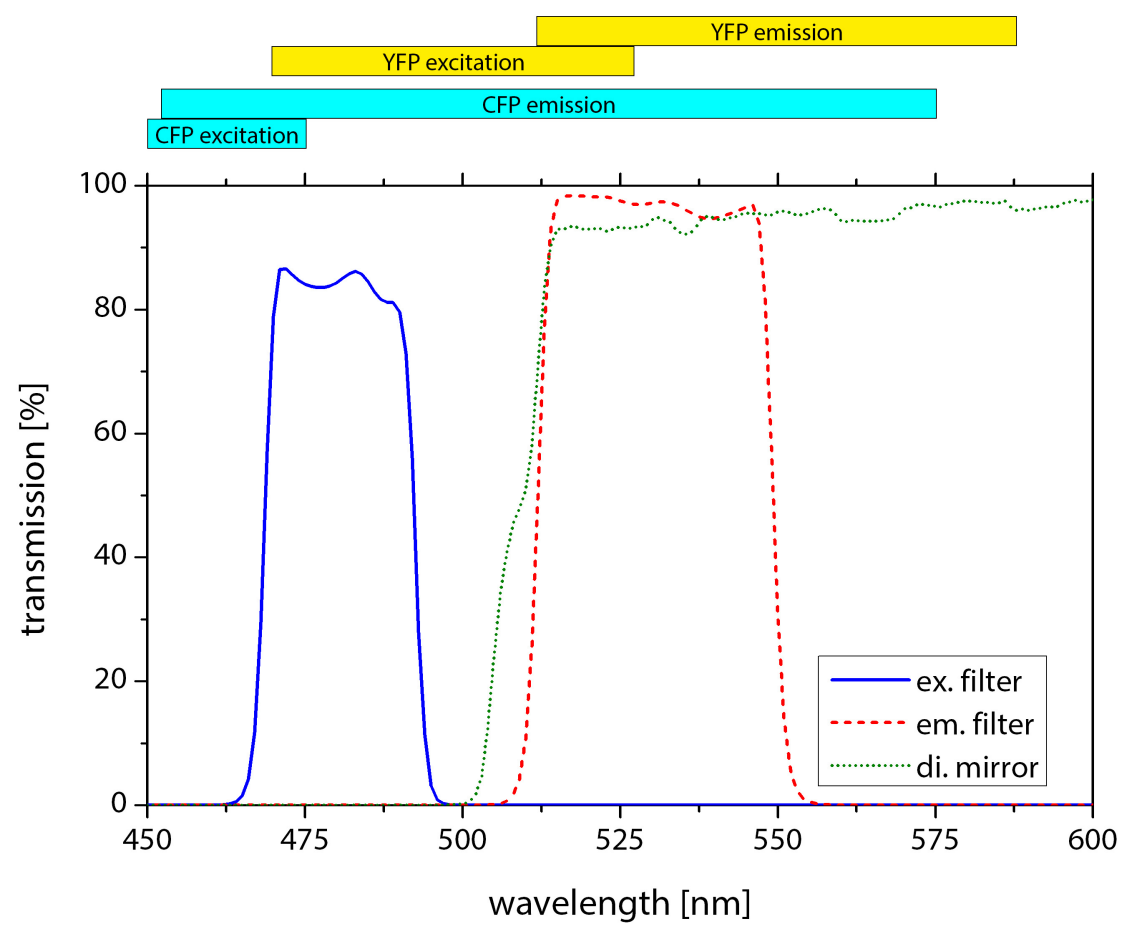

Figure 3.3: The excitation filter of the U-MNIBA3 filter-cube is transparent to light from 470 to $495 \mathrm{~nm}$, which is suitable for the combined excitation of fluorophores from CFPlabeling and in particular from YFP-labeling that are present in the keratin networks we observe in the SK8/18-2 cells (related excitation and emission wavelengths shown on top correspond to $20 \%$ of the relative intensity). The dichroic mirror and the emission filter are both transparent to light with a wavelength between 510 and $550 \mathrm{~nm}$ which is also in accordance with the spectra from the keratin labeling. Data taken from references [87, 88].

a consequence, the out-of-focus light, which consists of light from layers other than the focal plane and leads to blurring in recorded images, is reduced. To this end, the sample is illuminated with a laser beam focused to a single spot. In addition, there is a confocal pinhole in the optical pathway restricting light detection to a thin focal plane in the sample (see figure $3.1 \mathrm{~b}$ ). A full scale image is then acquired by scanning the sample with the laser beam while recording the sample emission, typically with a photomultiplier or a photodiode. For this purpose, the laser beam is deflected by scanning mirrors. The complete image is therefore assembled pixel by pixel from each scanning point in the sample. A disadvantage of this technique is, however, a longer duration of the acquisition process for each image (typically several seconds for the observation of SK8/18-2 cells) compared to the time needed to take an image with a common fluorescence microscope (typically $35 \mathrm{~ms}$ in our case). Furthermore, the bleaching effect is enhanced when using a confocal setup due to a longer total exposure time and a potential overlap of illumination spots in the sample, when choosing sufficiently small pixel steps. 
For image acquisition using a confocal setup, the Olympus IX 81 microscope is extended by an argon ion laser and an Olympus FV-1000 confocal unit. During confocal imaging, a DM405/488/461/633 excitation dichroic mirror (Olympus) was used in combination with a BA505IF emission filter (Olympus).

\subsection{Experiments with Cells under Shear-Flow}

Shear forces were applied to SK8/18-2 cells by microfluidic flow. In detail, we placed the SK8/18-2 cells in straight microfluidic channels (height $40.4 \mu \mathrm{m}$, width $150 \mu \mathrm{m}$ ). Since the cells in a suspension tend to clog inside tubings and especially at tight bendings, we cut one side of the microfluidic channel to be later able to insert the cells into the channel. The actual insertion of the cells into the microfluidic devices was performed directly after device fabrication through the opened ends of the channels. To achieve this goal, the cells were detached from the cell culture dish as described in section 3.1. Several drops of the resulting cell suspension with a concentration of about 1 million cells $\mathrm{ml}^{-1}$ were then placed to the ends of the channels at the cut edge (see figure 3.4 ). By establishing an under pressure with a syringe at the hole for tubing near the front end of the channels, the cell suspension was sucked through the channels. Afterwards, the device was completely covered with cell culture medium to prevent evaporation of the medium through the channel walls. The device was maintained in this state overnight in a cell incubator allowing the cells to reattach to the bottom surface of the channels and reequilibrate.

To establish a micro-flow the microfluidic devices were connected with polyethylene tubing (Intramedic PE20, Becton Dickins and Company, Franklin Lakes, USA) to gas-tight glass syringes ( $0.5 \mathrm{ml}$; Hamilton, Bonaduz, Switzerland). The syringes were previously loaded with cell culture medium, to which beads (diameter $500 \mathrm{~nm}$, 2\% solid diluted 1 : 50000; FluoSpheres; Invitrogen, Darmstadt, Germany) were added for visual control purposes. The syringes were operated by precise syringe pumps (neMESYS, Cetoni GmbH, Korbußen, Germany). Physiological conditions were meanwhile ensured by tempering the syringes with heat packs to $37^{\circ} \mathrm{C}$ in addition to the use of the incubation chamber (see 3.2). During shear-flow experiments, epifluorescence images were taken every $2 \mathrm{~s}$ with an exposure time of $35 \mathrm{~ms}$ employing a $100 \times, 1.4$ NA Olympus oil immersion objective (UPLSAPO 100XO). By using epifluorescence microscopy, it was possible to acquire a high number of frames before bleaching prevailed.

The experiment started with the recording of cells for 15 min in the absence of flow. The behavior of the keratin network during this time served both as a control 
and as a basis for the comparison with the behavior of the network under shear conditions. Afterwards, the flow was applied with a constant flow rate of $25 \mu \mathrm{l} \mathrm{h}{ }^{-1}$ for $20 \mathrm{~min}$. For the analysis, data of the first $5 \mathrm{~min}$ of flow conditions were neglected to exclude a unique network displacement from our analyses (see also chapter 4.3).

Both imaging of the SK8/18-2 cells and no-neighbor filtering were carried out using the xcellence software by Olympus.

\subsection{Microfluidic Devices}

The microfluidic devices which have been used for cell shearing experiments were made up of a molded silicon-based organic polymer PDMS (polydimethylsiloxane, $\left.\mathrm{CH}_{3}\left[\mathrm{Si}\left(\mathrm{CH}_{3}\right)_{2} \mathrm{O}\right]_{n} \mathrm{Si}\left(\mathrm{CH}_{3}\right)_{3}\right)$, which was covalently bonded to a glass cover slip. The material is very suitable for our purpose since it allows for the use of brightfield and fluorescence microscopy techniques due to its transparency to visible light $(240 \mathrm{~nm}$ to $1100 \mathrm{~nm}$ ). Furthermore, the usage of PDMS is convenient for experiments with cells: It is nontoxic and chemically inert. Additionally, the material is impermeable to liquid water on short timescales but permeable to gas and nonpolar organic solvents 89 .

The microfluidic device was produced using rapid prototyping [90]. First, a mask was designed using AutoCAD (Autodesk GmbH, München, Germany) software. The design was then commercially printed on a transparent foil by JD-Photo-Tools Ltd. (Oldham, UK) and used as a mask for photolithography [91 in a class 100 cleanroom. The production of the structured wafer which acts as a stamp for the PDMS elastomer was performed according to the photoresist data sheet 92. To this end, silicon wafers (SilChem, Freiberg, Germany) were cleaned with isopropanol and then dried both with nitrogen and by placing them on a hotplate with a temperature of $200{ }^{\circ} \mathrm{C}$ for 5 minutes. Afterwards, $1 \mathrm{ml}$ of SU-8 3025 (MicroChem, Newton, MA, USA) photoresist was poured on the wafer. Next, spin coating was started with a ramp from 0 to $500 \mathrm{rpm}$ (acceleration of $100 \mathrm{rpm} / \mathrm{s}$ ) and followed by a further ramp to a final speed of $2000 \mathrm{rpm}$ (acceleration of $300 \mathrm{rpm} / \mathrm{s}$ ). The final speed was maintained for $30 \mathrm{~s}$. After spin coating, the wafer with the thin layer of photoresist was soft-baked on a hotplate for $13 \mathrm{~min}$ at $95^{\circ} \mathrm{C}$. As depicted in figure 3.4 a, the photoresist was afterwards exposed to UV light $(\lambda=365 \mathrm{~nm})$ using a Karl Süss MJB4 mask aligner (Süss Microtech AG, Garching, Germany). Directly after exposure, the wafer was placed on a hotplate again for $1 \mathrm{~min}$ at $65^{\circ} \mathrm{C}$ followed by further 5 min at $95^{\circ} \mathrm{C}$. Finally, the resist was developed by placing and gently shaking the wafer in a developer solution (Microchem) for 10 min to wash away non-illuminated 
(a)

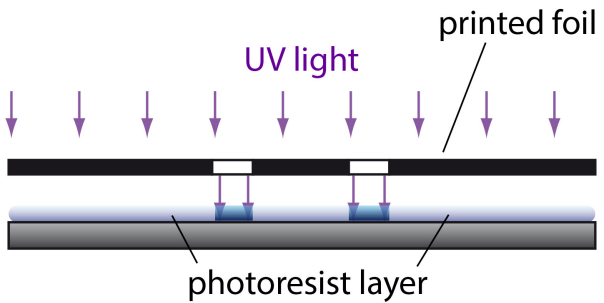

(c)

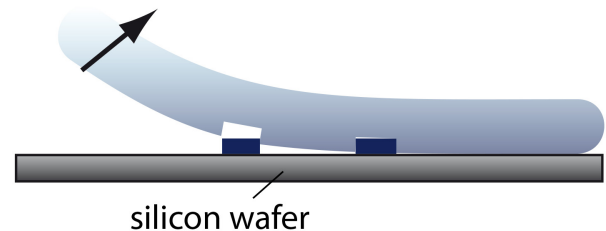

(e)

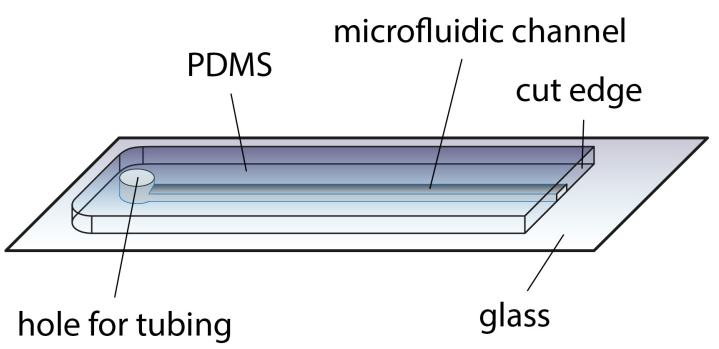

(b)

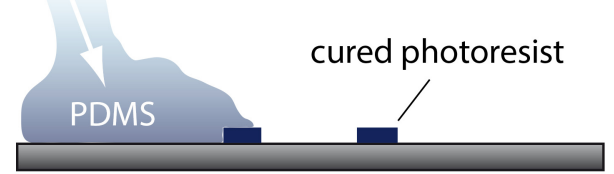

(d)

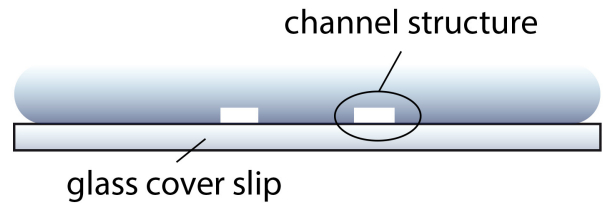

(f)

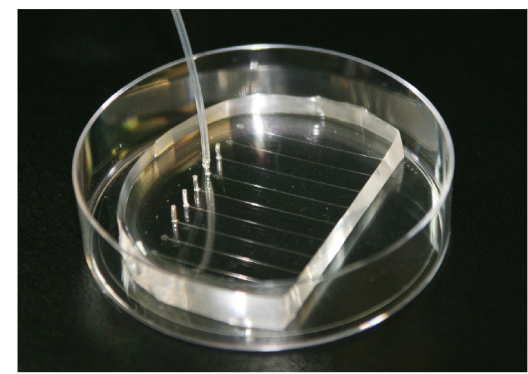

Figure 3.4: Side view of the microfluidic device fabrication: (a) Photo resist, which has been spin coated on top of a silicon wafer, was exposed to UV light through a printed mask and subsequently developed. (b) A PDMS cross-linker mixture was poured and cured on top of the developed photoresist structure so that the pre-cured, viscous PDMS was molded by the photoresist. (c) After curing in an oven, the PDMS cut along the edge of the wafer and subsequently was pealed off. (d) By exposing the elastomer to an oxygen plasma, it was finally covalently bonded to a glass cover slip. (e) Sketch of a final microfluidic device and a corresponding photo (f).

and thus non-cured photoresist. The height of the cured photoresist was determined using a profilometer (Dektak 6M, Veeco, Mannheim, Germany) and was measured to be $40.4 \mu \mathrm{m}$. To ensure a proper removal of the PDMS from the structure, the wafer was finally treated with (heptafluoropropyl)-trimethylsilane (Aldrich, Steinheim, Germany).

The wafer produced from the cured photoresist functioned as a stamp and allowed for the fabrication of multiple microfluidic devices. After placing the wafer in a petri dish, Sylgard 184 PDMS mixed with corresponding cross-linker at a ratio of 10:1 (Dow Corning GmbH, Wiesbaden, Germany) was poured on top of it (figure $3.4 \mathrm{~b})$. Subsequently, the PDMS-cross-linker mixture was degassed using a desiccator. The PDMS was then cured for $3 \mathrm{~h}$ in an oven with a temperature of $65^{\circ} \mathrm{C}$. 
Afterwards, the PDMS could be cut along the edge of the wafer and peeled off (figure 3.4.). As a following step, holes with a diameter of $0.75 \mathrm{~mm}$ for tubing connections were punched into the cured elastomer at one side of the channels using Harris Uni-Core biopsy punchers (Ted Pella, Inc, Redding, USA). Near the other side of the channels, the PDMS layer was cut with a scalpel for later insertion of the cell suspension. Eventual residue and dust were removed by cleaning both the PDMS and a glass cover slip with isopropanol. After drying the glass and the elastomer, they were exposed to an oxygen plasma for $10 \mathrm{~s}$ using a PDC-32G-2 plasma cleaner (Harrick Plasma, Ithaca, USA). During this process, methyl $\left(\mathrm{Si}-\mathrm{CH}_{3}\right)$ groups of the PDMS are substituted by silanol groups ( $\mathrm{Si}-\mathrm{OH}$ ) which changes the surface properties from hydrophobic to hydrophilic. Directly afterwards, both materials were brought into contact. This allows for the establishment of covalent bonds between the two surfaces ( $\mathrm{Si}-\mathrm{O}-\mathrm{Si}$; result is depicted in figure $3.4 \mathrm{~d}$ ).

\subsection{FEM Simulation}

Flow characteristics in the micro-channels were obtained by simulating the hydrodynamics using the software COMSOL Multiphysics (version 4.0 to 4.4; COMSOL Multiphysics GmbH, Göttingen, Germany). The software is able to describe and solve physical problems based on partial differential equations by employing finite element methods (FEM). This numerical approach comprises subdividing the object of interest into a finite amount of elements, for which a solution is then approximated in accordance with user defined starting, boundary, and appropriate element-transition conditions. Since shear stress depends on the channel height, we performed three dimensional simulations of the flow field for flow characterization. The basis for the simulation of the fluid motion inside microfluidic channels was the Stokes equation (see chapter 4.1.1). As flow material, water was chosen at a temperature of $37^{\circ} \mathrm{C}$. The physical properties of water, like the viscosity or density, were taken into account accordingly. For the channel walls and the cell-like obstacle in the channels, no-slip boundary conditions were chosen. The inlet was defined as a cross-section with a volumetric flux of $25 \mu \mathrm{lh}^{-1}$. Here, COMSOL allows for a definition of an entrance length ( $1 \mathrm{~m}$ as a default value) so that the flow distribution at the inlet is no longer homogeneous but has the equilibrated profile of an extended channel. 


\subsection{Buckling-Experiments}

To record buckling events of keratin bundles, we built microscopy-compatible cell chambers. To this end, holes were drilled into the center of normal cell culture dishes. In a next step, glass cover slips were glued beneath the hole using a silicone glue (Scrintec 600, Roth, Karlsruhe, Germany). After curing for $24 \mathrm{~h}$, the cell chambers were sterilized by exposure to UV light in a safety cabinet (Herasafe Class II, Thermo Fisher Scientific, Inc, Waltham, MA, USA). Afterwards, the cells were seeded on the glass surface as described for normal cell passaging (see section 3.1). Subsequently, they were kept overnight in a cell incubator.

To ensure high resolution images, where the bundle shape can be resolved properly, the keratin network containing cells were observed using confocal microscopy. For sample illumination, a $488 \mathrm{~nm}$ laser-line of an argon-ion laser was used with an intensity of 0.1 to $2 \%$. To reduce the out-of-focus light, the pinhole diameter was adjusted to the smallest value possible, i.e. $101 \mu \mathrm{m}$. The images were assembled of pixels which had each an illumination time of $2 \mu \mathrm{s}$. The step width between the pixels was chosen to be within a range of 20 to $80 \mathrm{~nm}$. 


\section{Cells in Micro-Flow}

This chapter describes our work on the shear stress response of keratin networks in live cells as revealed by epifluorescence microscopy recordings (see also section 3.3). Shear stress conditions are established using microfluidic methods. In particular, we characterize the flow-field and shear stress distribution in the microfluidic channels by providing both the theoretical background for fluid dynamics as well as a description of performed simulations and their results in section 4.1. Having described shear stress generation, we present the methods used for data analysis in section 4.2 . These methods contain a global approach in which images are cross-correlated to yield typical time scales for the motion in the overall depicted network region. In addition, we develop and employ methods, in which selected network nodes are locally tracked and the spatial spreading and temporal evolution of the trajectories are quantified. The obtained results are shown in section 4.3 together with our findings regarding the effect of myosin II motor protein inhibition on keratin network motion. In the subsequent section 4.5 we finally discuss implications our data suggest for the origin of keratin network dynamics, for the involvement of cytoskeletal cross-talk and for a cellular short-term adaptation mechanism to protect the cell against external forces.

Parts of this chapter have been published in New Journal of Physics [93.

\subsection{Fluid Dynamics}

The following section contains a review of fluid dynamics specifying important equations of motion and simplifications that apply to the special case of microfluidics. An in-detail description of hydrodynamics in microfluidics is given in reference 94. The equations for microfluidics are further implemented in a simulation of the flowfield inside a channel which contains a cell-shaped obstacle. The simulations enable 
a characterization of the flow-induced shear stress which is experienced by the cells in our measurements.

\subsubsection{Theoretical Background}

The Navier-Stokes-Equation (NSE) is the basic equation describing fluid dynamics. In generalized form the NSE for Newtonian and incompressible fluids (e.g. water) is given by the following relation 4.1 :

$$
\rho(\underbrace{\frac{\partial \vec{u}}{\partial t}}_{\begin{array}{c}
\text { local } \\
\text { acc. }
\end{array}}+\underbrace{(\vec{u} \cdot \nabla) \vec{u}}_{\begin{array}{c}
\text { convective } \\
\text { acc. }
\end{array}})=\underbrace{-\nabla p}_{\begin{array}{c}
\text { pressure } \\
\text { gradient }
\end{array}}+\underbrace{\eta \nabla^{2} \vec{u}}_{\begin{array}{c}
\text { viscous } \\
\text { forces }
\end{array}}+\underbrace{\vec{f}}_{\begin{array}{c}
\text { external } \\
\text { forces }
\end{array}} .
$$

On the left hand side of the NSE the inertial forces 1 are shown which describe the local and non-linear convective acceleration of the fluid, where $\rho$ represents the density, $\vec{u}=(u, v, w)=\frac{\mathrm{d} \vec{x}}{\mathrm{~d} t}$ the velocity and $t$ the time. These inertial forces are balanced by body forces which consist of a pressure gradient, viscous and potentially also external forces.

Fluids are called Newtonian if the shear stress $\sigma$ is proportional to the strain rate $\dot{\gamma}$ which is the change of the fluid velocity $\dot{\gamma}=\frac{\partial u}{\partial z}$ in units of $1 /$ s (see figure 4.1), i.e.

$$
\sigma=\eta \cdot \frac{\partial u}{\partial z}
$$

Here, the constant of proportionality $\eta$ between shear stress and strain represents the dynamic viscosity of the fluid.

The NSE can also be expressed in dimensionless form. To this end, characteristic values for the length $(L)$ and velocity $(U)$ are introduced and the parameters in

\footnotetext{
${ }^{1}$ more precisely: forces per unit volume
}

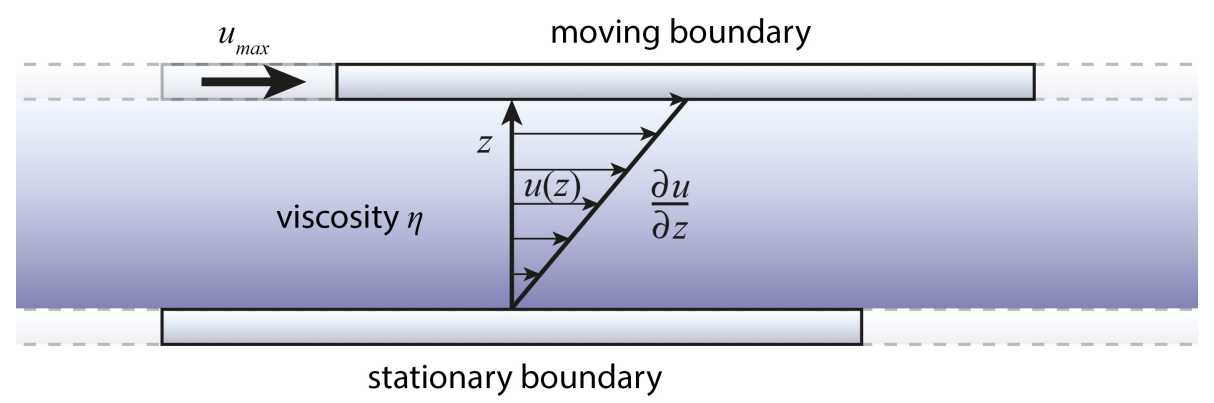

Figure 4.1: When moving one boundary plane against another, the resulting velocity gradient $\frac{\partial u}{\partial z}$ is proportional to the shear stress $\sigma$ for Newtonian fluids. The proportionality factor is given by an intrinsic property of the fluid: the dynamic viscosity. 
equation 4.1 are transformed in the following way:

$$
\begin{aligned}
& \vec{x}=L \tilde{\vec{x}} \\
& \vec{u}=U \tilde{\vec{u}} .
\end{aligned}
$$

Characteristic values for the time $(T)$ and the pressure $(P)$ furthermore yield

$$
\begin{aligned}
t & =\frac{L}{U} \tilde{t}=T \tilde{t} \\
p & =\frac{\eta U}{L} \tilde{p}=P \tilde{p} .
\end{aligned}
$$

At this stage, we would like to point out that $L, U, T$ and $P$ represent dimensionassociated constants, which are solely introduced to arrive at a dimensionless NSE. They are hence unaffected by derivatives. By inserting the above characteristics into the NSE under exclusion of external forces and by afterwards using the chain rule for the derivatives $\partial_{t}=\frac{\partial}{\partial t}=\frac{\partial \tilde{t}}{\partial t} \frac{\partial}{\partial \tilde{t}}=(1 / T) \tilde{\partial}_{\tilde{t}}, \nabla=(1 / L) \tilde{\nabla}$ we therefore obtain:

$$
\begin{aligned}
\rho\left(\frac{U}{T} \partial_{\tilde{t}} \tilde{\vec{u}}+\frac{U^{2}}{L}(\tilde{\vec{u}} \cdot \tilde{\nabla}) \tilde{\vec{u}}\right) & =-\frac{P}{L} \tilde{\nabla} \tilde{p}+\frac{\eta U}{L^{2}} \tilde{\nabla}^{2} \tilde{\vec{u}} \\
\Rightarrow \rho\left(\frac{U^{2}}{L} \partial_{\tilde{t}} \tilde{\vec{u}}+\frac{U^{2}}{L}(\tilde{\vec{u}} \cdot \tilde{\nabla}) \tilde{\vec{u}}\right) & =-\frac{\eta U}{L^{2}} \tilde{\nabla} \tilde{p}+\frac{\eta U}{L^{2}} \tilde{\nabla}^{2} \tilde{\vec{u}} \\
\Rightarrow \operatorname{Re}\left(\partial_{\tilde{t}} \tilde{\vec{u}}+(\tilde{\vec{u}} \cdot \tilde{\nabla}) \tilde{\vec{u}}\right) & =-\tilde{\nabla} \tilde{p}+\tilde{\nabla}^{2} \tilde{\vec{u}} .
\end{aligned}
$$

In equation 4.9 the Reynolds number Re was introduced with

$$
R e:=\frac{\rho U L}{\eta}
$$

The Reynolds number represents the ratio between the inertial forces and the viscous forces, and characterizes the flow. For small dimensions (or small flow velocities or large fluid viscosities) the viscous forces dominate $(R e \ll 1)$. In such cases, the inertial forces can be neglected and the NSE can be approximated by the following equation:

$$
\rho \partial_{t} \vec{u}=-\nabla p+\eta \nabla^{2} \vec{u}
$$

Considering stationary flow, where the flow field is independent of time, we obtain the Stokes-equation:

$$
0=-\nabla p+\eta \nabla^{2} \vec{u}
$$


Particular for microfluidic methods is a Reynolds number which is smaller than one. In fact, by inserting typical values for our microfluidic setup (water flow in a channel with a height of $40 \mu \mathrm{m}$ and a flow velocity of $1 \mathrm{~mm} / \mathrm{s}$ ) into equation (4.10), we arrive at a Reynolds number of 0.04. Furthermore, the flow in our experiments is time-independent since the velocity is kept constant. Characteristic for hydrodynamics on sub cellular length scales is a low Reynolds number which indicates a flow that is always laminar, i.e. non-turbulent 95]. For our time-independent purpose, the Stokes-equation applies.

\subsubsection{Flow-Fields in Microfluidic Channels}

Although the nature of the Stokes-equation is simpler - especially as a result of the missing convective term $(\vec{u} \cdot \nabla) \vec{u}$ - an exact analytical solution of the equation is not available for rectangular channels. Hence, FEM simulations (see section 3.5) have been performed to access the flow conditions. Apart from simulating the flow-field through obstacle-free rectangular channels, we have also studied the changes which are caused by a cell-shaped object attached to the bottom of the channels using FEM methods. For this latter simulation, we have derived the shape of the cell-like obstacle from 3D confocal microscopy image stacks of 12 cells. The cell surface of each cell, which has been extracted from the outer contour of the keratin network region, shows a geometry of spherical caps. Here, the radius of the averaged cap was determined to be $R=23.6 \mu \mathrm{m}$, its height $h_{\mathrm{c}}=8.8 \mu \mathrm{m}$ and its footprint radius $r_{\mathrm{c}}=18.4 \mu \mathrm{m}$ (compare figure $4.2 \mathrm{~b}$ ) 96 .

Figure 4.2 depicts the results of the simulated three dimensional flow-field inside a microfluidic channel (width $w=150 \mu \mathrm{m}$, height $h=40 \mu \mathrm{m}$ ) containing a cell-like obstacle and a constant fluid influx of $Q=25 \mu \mathrm{l} / \mathrm{h}$ at the inlet. For the simulation the object was placed at the center of the channel. As shown in the figure, the flow is changed by the presence of the cell. On the one hand, the cell narrows down the cross-sectional area of the channel (at maximum $A_{\text {channel,nocell }}=6000 \mu \mathrm{m}^{2}$ vs. $A_{\text {channel,nocell }}=5774 \mu \mathrm{m}^{2}$ ) so that the mean velocity is increased, however only slightly, from $\left\langle v_{\text {nocell }}\right\rangle=A_{\text {channel,nocell }} / Q=1.16 \mathrm{~mm} / \mathrm{s}$ to $\left\langle v_{\text {cell }}\right\rangle=A_{\text {channel, withcell }} / Q=$ $1.20 \mathrm{~mm} / \mathrm{s}$. On the other hand and more noticeably, the flow profile is altered.

These aspects become very apparent, for example, in figure 4.3 . Here, the flow behavior is shown with respect to the $z$-axis at the center of the channel. While the maximum value of the velocity is nearly constant along this axis, the slope of the velocity changes especially in the region directly above the cell. Since the combination of the viscosity of the fluid and the velocity derivative defines the shear stress (see equation (4.2)), it is changed by the presence of the cell due to changes 
(a)

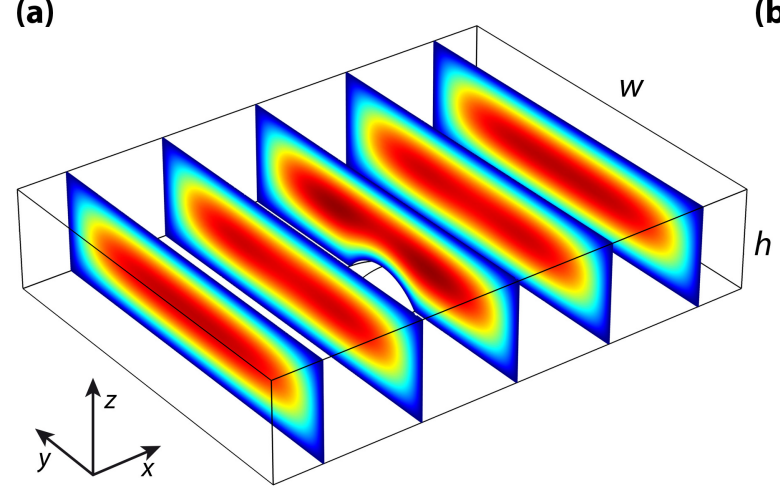

(b)
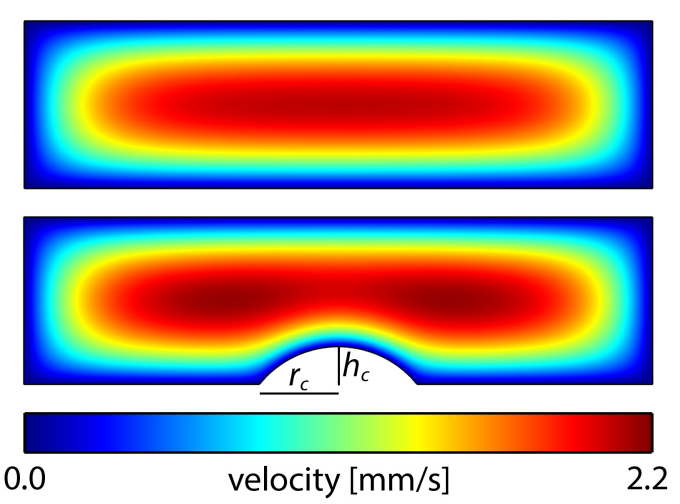

(d)
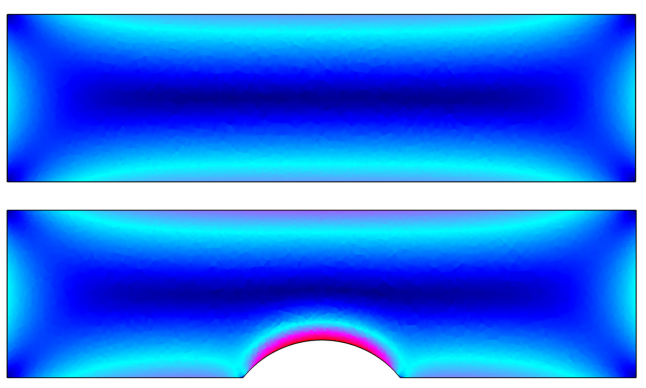

0.0

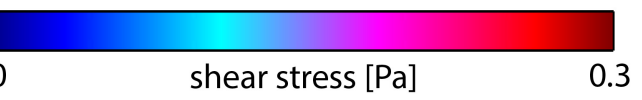

Figure 4.2: Flow-field simulation for a rectangular channel (width $w=150 \mu \mathrm{m}$, height $h=40 \mu \mathrm{m}$ ) containing a spherical cap as a solid obstacle at the central bottom surface (height $h_{\mathrm{c}} \approx 9 \mu \mathrm{m}$, footprint radius $r_{\mathrm{c}} \approx 18 \mu \mathrm{m}$ ). The volumetric flow inside the channel was set to $Q=25 \mu \mathrm{l} / \mathrm{h}$ according to flow rates used in experiments. (a) $3 \mathrm{D}$ overview of the flow velocity. The flow direction is along the $x$-axis. (b) $2 \mathrm{D}$ cross-sections showing the velocity as a function of $y$ and $z$ in a comparison for the two situations with and without a cell. (c) Shear stress distribution inside the channel. (d) Shear stress calculation corresponding to subfigure (b). In the presence of a cell, the stress at the corresponding boundary is increased by nearly a factor of 2 . 
(a)

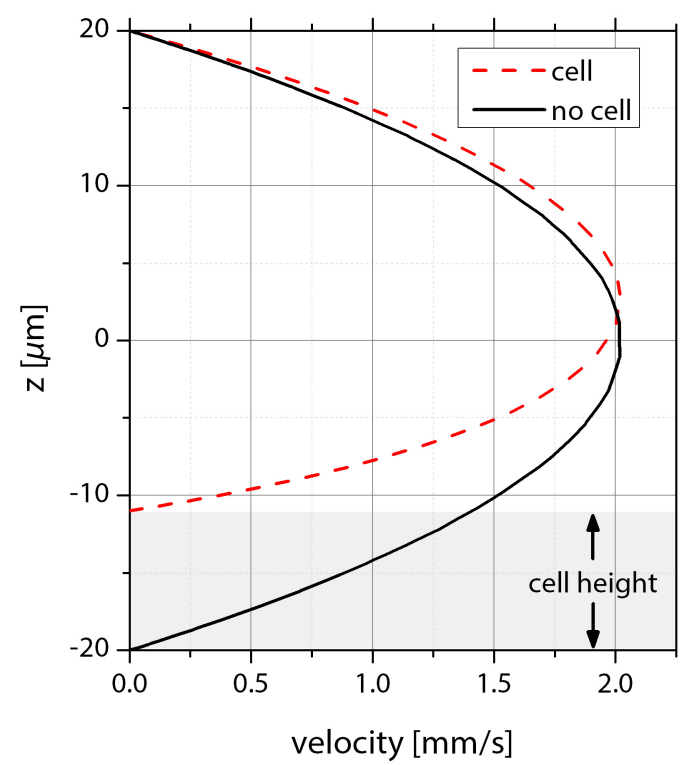

(b)

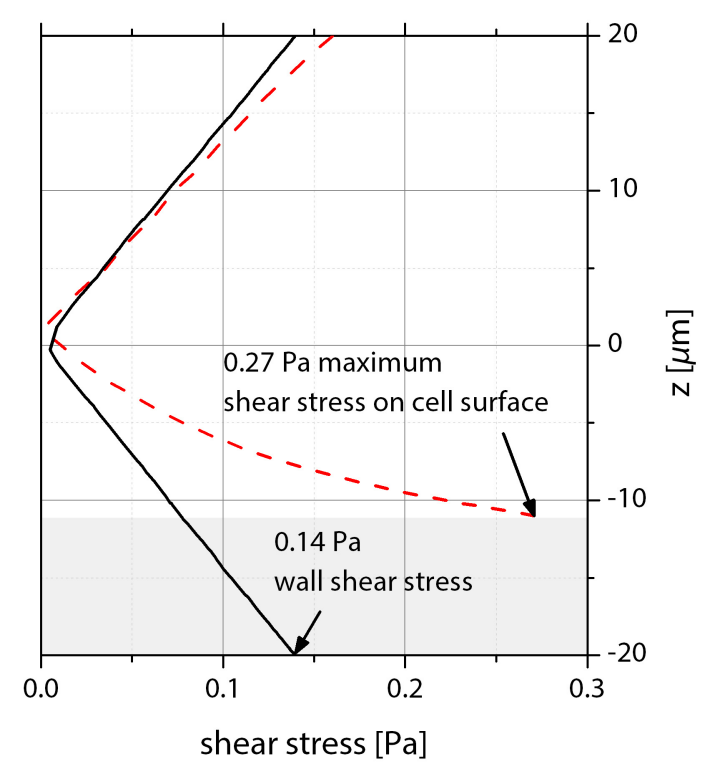

Figure 4.3: One dimensional representation of the flow field along the $z$-axis at the center of the channel both in the presence and in the absence of a cell. (a) The maximum velocity is nearly independent of the presence of the cell at this position in the channel. (b) The shear stress distribution, however, shows a distinct change of its maximum value. The stress on top of the cell is twice the stress at the channel wall in the cell-free setup.

of the flow velocity. Instead of a maximum shear stress of $0.14 \mathrm{~Pa}$ at the wall of cell-free channels, we now obtain a value that is nearly doubled to $0.27 \mathrm{~Pa}$ directly on top of the cell (straight black versus dashed red curve in figure 4.3).

For the analysis of the effect of shear stress on cells, we have performed experiments on many days using several microfluidic devices and studying a number of cells of the same cell line. To guarantee comparability between the different recorded data sets it was therefore necessary to establish reproducible starting conditions. Here, we found that choosing a channel width of $150 \mu \mathrm{m}$ is suitable to that goal. As depicted in figure 4.4, there is a nearly constant shear stress region in the middle of the channel. In fact, the shear stress varies only by $4 \%$ within half of the channel width (area covering $1 / 4$ to $3 / 4$ of the channel width). Thus, we identified the central region within the channels to be appropriate for the study of our cells, since the cells that are located in this region encounter similar shear stress conditions. Very similar shear conditions, in turn, lead to comparable data which is essential for gathering appropriate statistics. 


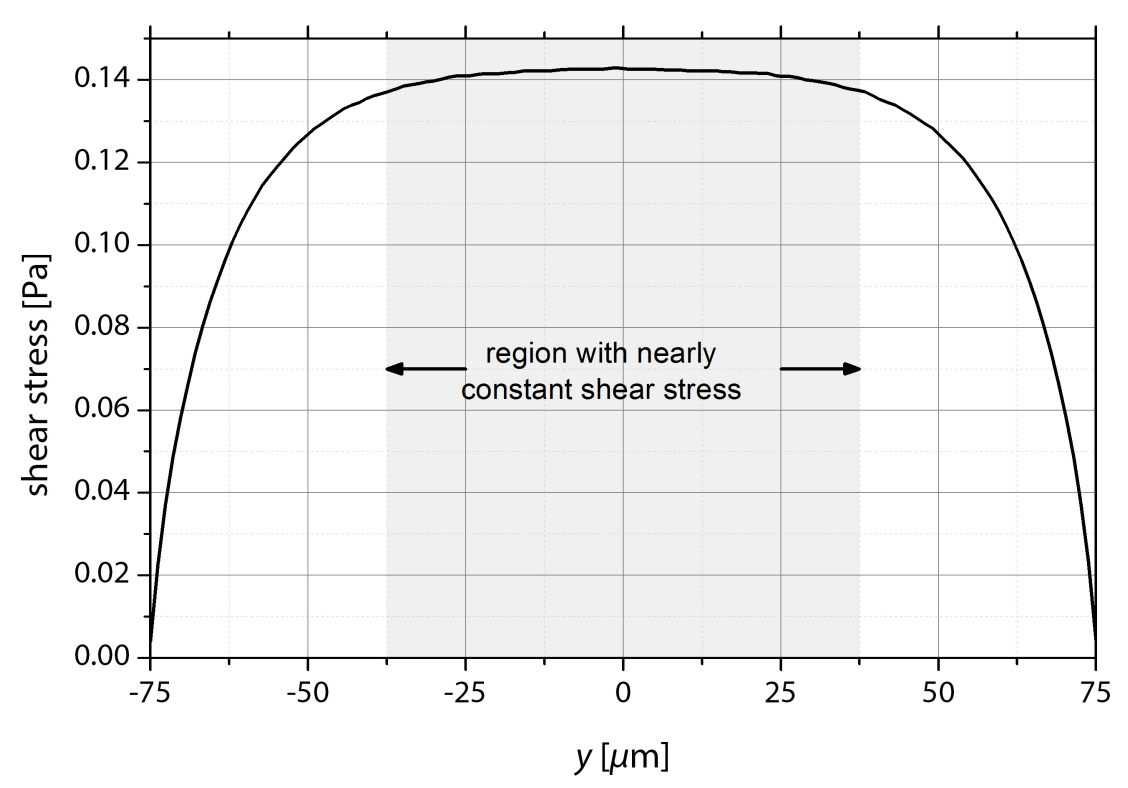

Figure 4.4: Shear stress at the bottom surface of the channel plotted against the axis along the channel width. In a region centered around the middle of the channel and covering half of the channel width, the shear stress distribution is nearly constant and thus it represents a suitable location for cells to be studied.

\subsection{Data Analysis of Keratin Networks in Cells}

After acquiring images of the keratin networks with epifluorescence microscopy, the recordings were processed to reduce noise and enhance contrast (section 4.2.1). The images obtained were then used for the analysis of the keratin network dynamics. To this end, we followed two approaches: As a global approach, where the whole network is characterized simultaneously, image-to-image cross-correlations were performed (section 4.2.2). This approach has the advantage that local variations in keratin bundle behavior are directly averaged out. Thus, only one characteristic parameter for the whole network is obtained. The aforementioned advantage can be, however, also seen as a disadvantage since information about the network motion on small scales is lost. To complete the picture, we therefore complemented the global crosscorrelation approach with an analysis of network node trajectories to also study local effects of shear stress (section 4.2.3). For both approaches, we focused on a region in the cell below the nucleus. In this region, the motion of the network in $z$-direction is reduced by the confinement caused by the nucleus on top and the glass coverslip from below. As a result the network motion is restricted to a $2 \mathrm{D}$ plane thus ensuring that the network remains in focus over the time course of our experiments. In addition, the KIF network located below the nucleus undergoes 
less reorganization and remains stable for a longer period in time as compared to the network at the cell periphery [71. This, in turn, guarantees comparability of the recorded data over the entire duration of the experiments. Finally, the image quality is enhanced below the nucleus due to the circumstance, that the nucleus is keratin-free and there is hence less out-of-focus light blurring the images.

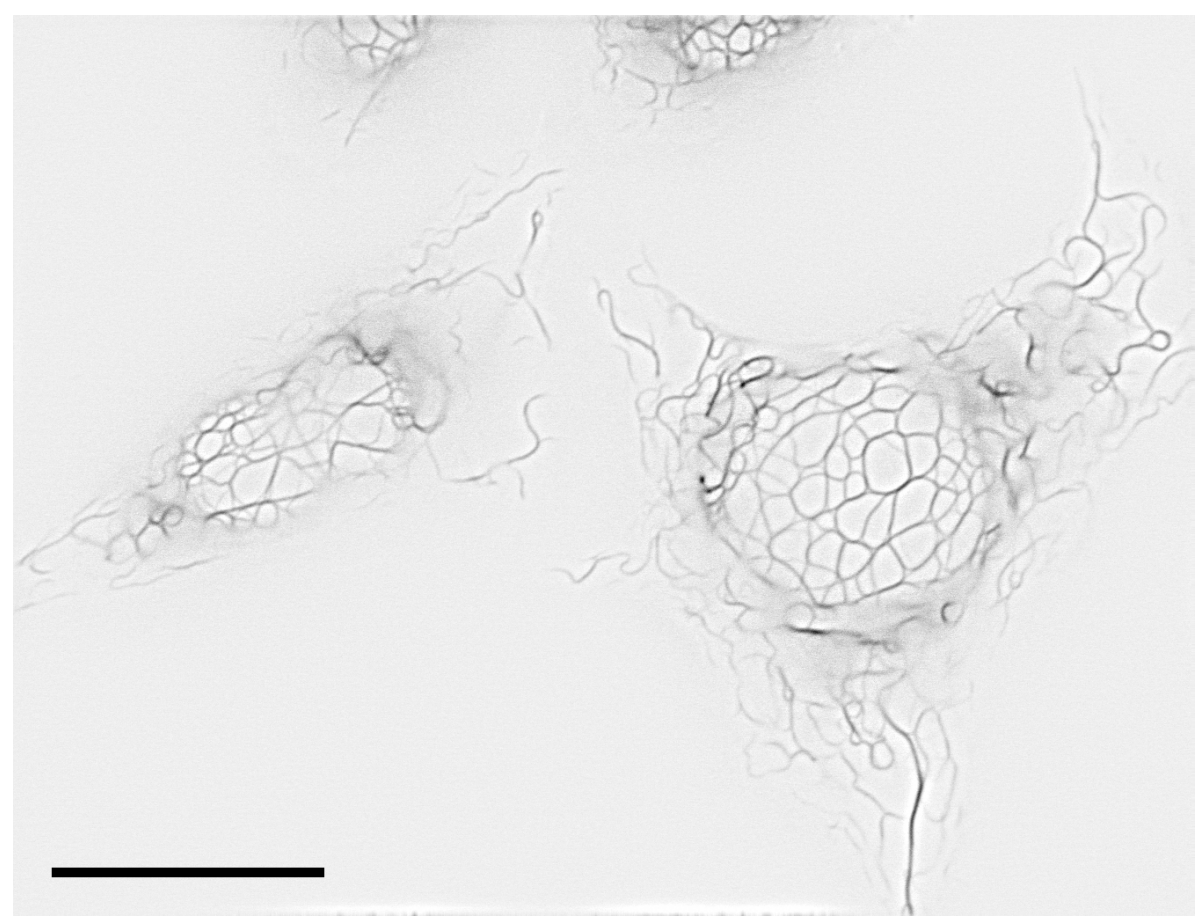

Figure 4.5: Inverted but otherwise unprocessed epifluorescence microscopy image of SK8/18-2 cells inside a microfluidic channel (not visible). Scalebar corresponds to $20 \mu \mathrm{m}$.

\subsubsection{Image Preprocessing}

The recorded 16-bit grayscale image stacks were preprocessed using Matlab (version 7.9.0.529 (R2009b) with Image Processing Toolbox; The MathWorks, Natick, MA, USA). For this purpose, a script was written which makes use of Matlab image filters. During this step, a median, a Wiener and a Gaussian filter were applied to every frame. The median filter is a non-linear filtering technique which is able to reduce noise but preserves edges. Within a defined region (in our case a $3 \times 3$ matrix) of each pixel a median value is calculated which replaces the original pixel, which is especially useful to reduce so-called salt-and-pepper noise. A similar effect is obtained when using the Wiener filter. This filter is based on a low-pass filtering technique. The final Gaussian filter is responsible for removing the remaining noise in the image. After noise removal, the grayscale values of the image were processed to enhance the contrast. To this end, the grayscale values were changed in a way 
as to have $1 \%$ of the pixels saturated at the low and the high end of the possible grayscale range, respectively. After this process which is basically a stretching of the histogram, all possible grayscale values are used which simplifies the identification of the node positions for example. The result of the preprocessing is depicted in a comparison of an original and a final image in figure 4.6.

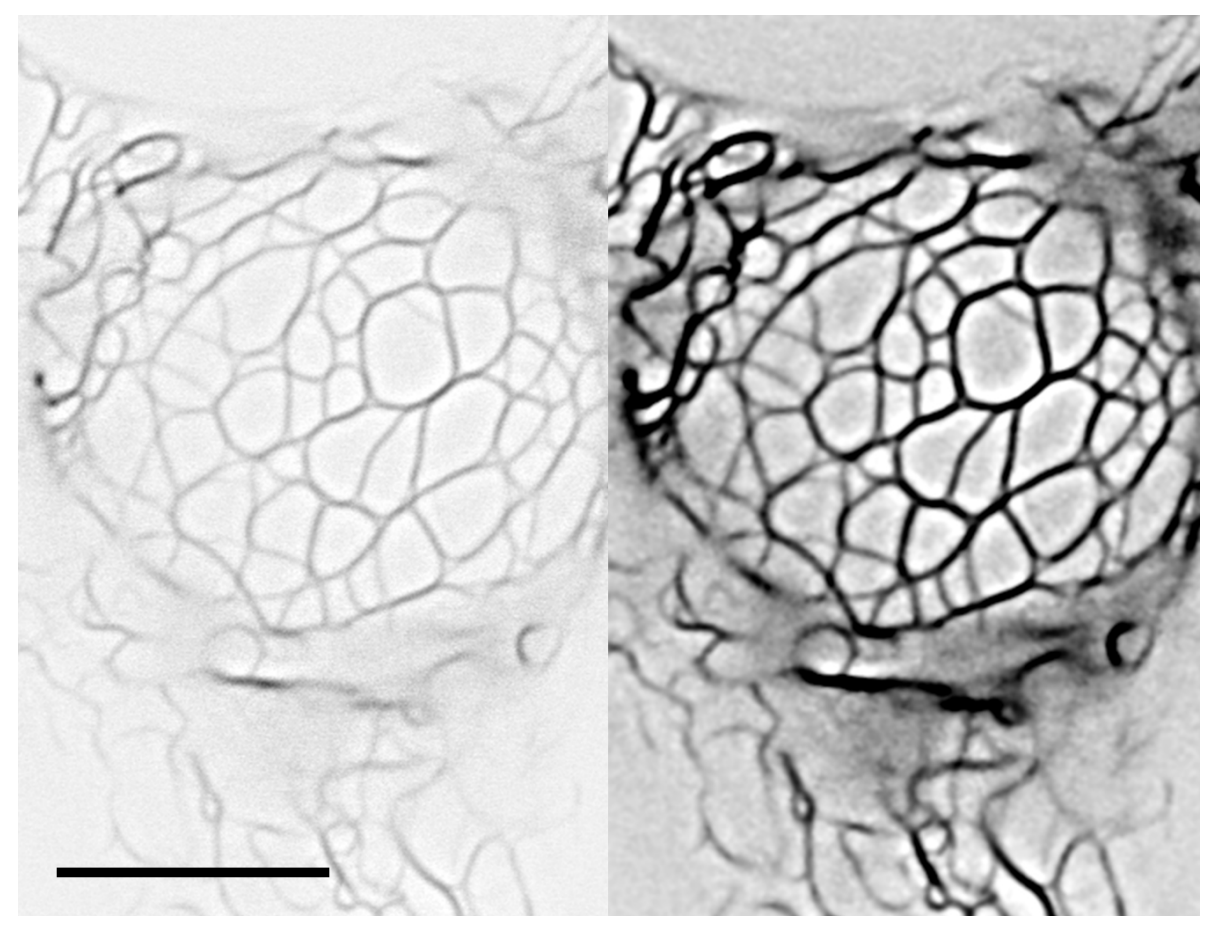

Figure 4.6: Comparison between an inverted raw and an inverted processed epifluorescence microscopy image of a SK8/18-2 cell. Image processing consisted of an automatic contrast adjustment, and a combination of a Wiener, median and Gaussian filter (see text for details). Scalebar corresponds to $10 \mu \mathrm{m}$.

\subsubsection{Image-to-Image Cross-Correlations}

As a global approach to characterize the dynamics of keratin networks in live cells we applied a correlation to our images twice. First, we performed a spatial correlation to correct for drift or overall cell motion in the recorded data. Secondly, we calculated a temporal correlation to obtain the characteristic time scales for the bundle motion in the keratin network. The procedure was carried out for the data sets for no-shear and shear conditions separately to compare the obtained results and conclude upon shear-induced changes in the keratin network dynamics.

In more detail, the correlation between two signals is basically a measure for the similarity between the signals - in the context of this work two image frames separated by a time shift $\tau$. The correlation values plotted against the time shift $\tau$ 
constitute the correlation function. In principle, for any two image frames taken one right after the other, the network has typically moved only little in between frames and the images tend to resemble one another. Hence, the correlation function for small $\tau$ yields high values. The larger the time difference between any two frames, the more the network moves and the less likely the images look alike. Therefore, the correlation function decreases for large time shifts, usually following an exponential decay. The decay time is then indicative of the typical time scales of the network dynamics.

In mathematical terms, a simple correlation between the signals $f(x)$ and $g(x)$ is defined by

$$
C C\left(x^{\prime}\right):=\frac{\int f(x) \cdot g\left(x+x^{\prime}\right) \mathrm{d} x}{\int \mathrm{d} x},
$$

Here, the correlation is calculated between the two functions with respect to their common parameter $x$. If $f$ and $g$ are two separate functions, the correlation is termed a cross-correlation, whereas if $g$ is replaced by $f$ the correlation is known as an auto-correlation function. For our case of an image-to-image correlation, the expression above becomes more complex. First, the two functions $f$ and $g$ are replaced by an image $I_{t}$ and an image at another point in time $I_{t+\tau}$. The common parameters for the two images are then the dimensions of space: $x$ and $y$. Seeing that our two functions are two distinct images in time, while the integral is performed over the coordinates in space, we refer to the correlation as a $2 \mathrm{D}$ cross-correlation (CC). The expression for the $\mathrm{CC}$ is then given by

$$
C C\left(t, t+\tau, x^{\prime}, y^{\prime}\right)=\frac{\iint I_{t}(x, y) \cdot I_{t+\tau}\left(x+x^{\prime}, y+y^{\prime}\right) \mathrm{d} x \mathrm{~d} y}{\iint \mathrm{d} x \mathrm{~d} y} .
$$

As a result we obtain a 2D map of the correlation as a function of $x^{\prime}$ and $y^{\prime}$. We are interested in the value of the maximum of the correlation and its location:

$$
C C_{\max }(t, t+\tau)=\max _{x^{\prime}, y^{\prime}}\left(C C\left(t, t+\tau, x^{\prime}, y^{\prime}\right)\right) .
$$

The coordinates for which the CC is maximal are also used to compensate for drift in the images (e.g. cell locomotion, or drift of the microscope stage) which is important when investigating node trajectories (see section 4.2.3). The maximum of the $\mathrm{CC}$ is then normalized so that it is equal to 1 when the image $I_{t+\tau}$ is identical to the original one $I_{t}$ except for overall intensity changes. Overall intensity changes result mostly from bleaching of the fluorescent dyes. As bleaching is also characterized by typical decay times, it contributes to the decay of the CC before normalization. Our normalization was therefore chosen in such a way as to yield a value of 1 for the entire CC in case of a fully immobilized network showing only bleaching effects. In 
all other cases of a moving network in living cells, our form of normalization removes the contribution of bleaching to the CC so that the normalized CC contains solely information about the typical time scales of the network motion.

$$
\widetilde{C C}_{\max }(t, t+\tau)=\frac{C C_{\max }(t, t+\tau)}{\sqrt{C C_{\max }(t, t) \cdot C C_{\max }(t+\tau, t+\tau)}} .
$$

The described calculation is performed for every time shift and averaged, yielding the final expression:

$$
C C(\tau):=\frac{\int \widetilde{C C}_{\max }(t, t+\tau) \mathrm{d} t}{\int \mathrm{d} t} .
$$

Image-to-image correlations according to equation 4.15 were calculated with sub-pixel accuracy (1/100 pixel) using a Matlab program from Guizar-Sicairos et al. [97]. The script takes advantage of a mathematical concept by calculating the cross-correlation in Fourier space. In Fourier space a convolution turns into a simple element-wise matrix multiplication. That is, in our case the Fourier-transformed images were multiplied: $\mathcal{F}\{f * g\}=\mathcal{F}\{f\} \cdot \mathcal{F}\{g\}$ or $f * g=\mathcal{F}^{-1}\{\mathcal{F}\{f\} \cdot \mathcal{F}\{g\}\}$. To further enhance computing efficiency some datasets were calculated using an adapted script which enables GPU computing.

\subsubsection{Characterization of Node-Trajectories}

For the analysis of keratin network node trajectories, the position of a node was extracted from the microscopy images. To this end, we mainly followed two approaches. At first, we aimed for a method to detect the node positions automatically. To accomplish this goal a small region of interest around a network node was defined in the first image and by searching for the maximum of the cross-correlation in the next frame (as described in section 4.2.2) the node position was determined for the new frame. For all subsequent frames the procedure was repeated taking the first frame of any two neighboring frames as a reference to calculate the cross-correlation for the next frame. We opted for this adaptive cross-correlation method, instead of fixing the first time frame as a reference for all correlation functions, to account for morphological changes occurring over the time course of our recordings. This method was tested very successfully, even for sub-pixel steps, for a 2D Gaussian test pattern which was moving along a predefined path. However, the tracking of nodes in the keratin network of cells turned out to be more challenging. Firstly, the network was not fixed in one plane and still showed small movement in $z$, even though the motion in this dimension was limited by the glass surface and the cell nucleus. Secondly, the aforementioned changes in keratin node morphology reduced 

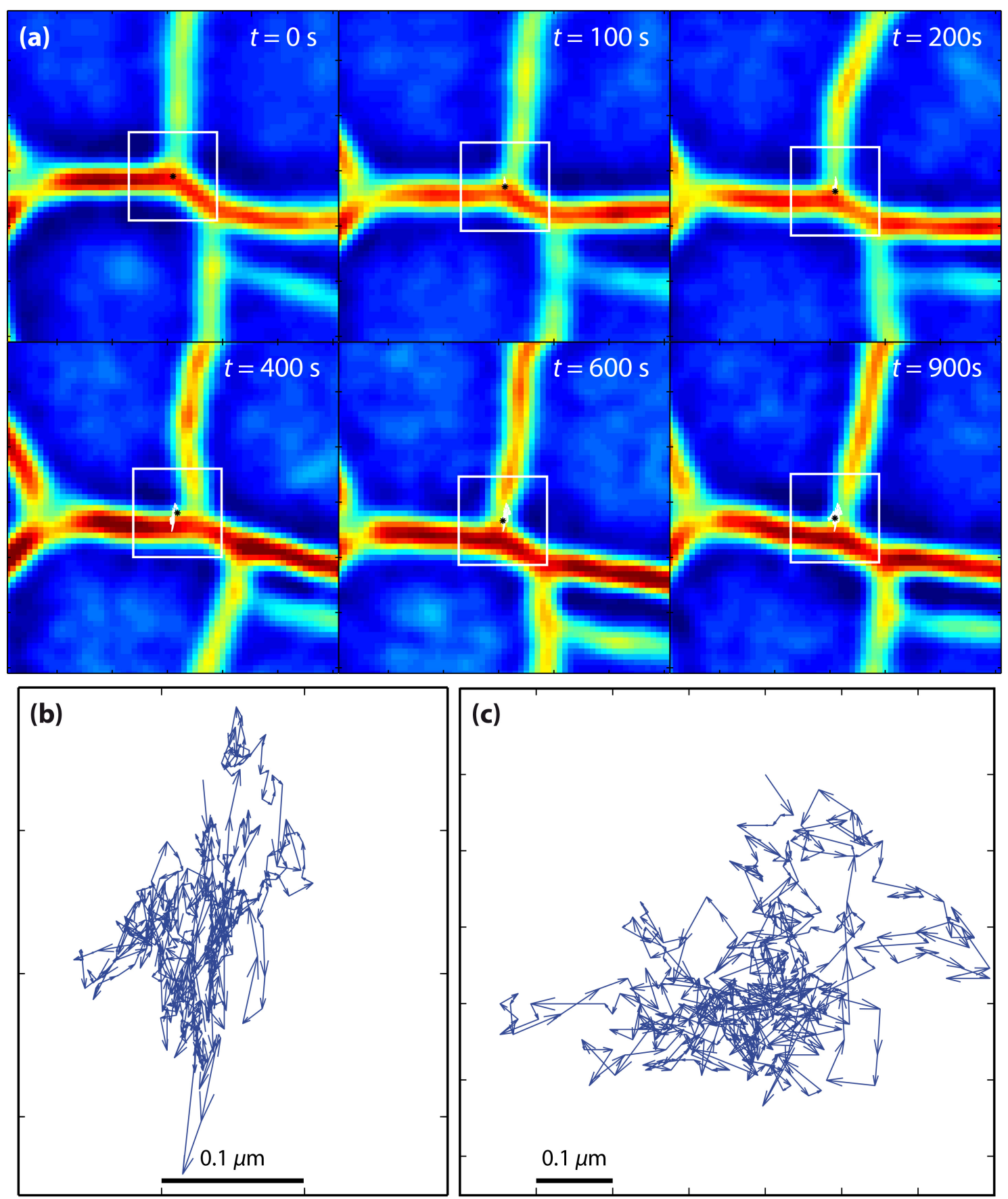

Figure 4.7: In the first image of a recorded data set, one or multiple nodes of the network are marked manually which are then tracked using $2 \mathrm{D}$ cross-correlations. The result of tracking for an example network node is shown in subfigure (a) following the temporal progress in detail (edge length $\sim 4 \mu \mathrm{m}$ ). Here, the white box marks the correlation window (16 pixels in this example). Already after 50 frames $(100 \mathrm{~s})$ the center of the node is lost to some degree. This error amplifies over time. After 200 frames $(400 \mathrm{~s})$ the distance between the center of the node and the detected one amounts to several pixel. The trajectory obtained from the automatic tracking (b) differs distinctly from the manual approach (c). The automatically obtained trajectory suggests a less pronounced motion of the node. However, the basic structure of the node trajectory is captured by both approaches. Thus, for example the loop at the bottom right appears in both plots. 
the maximum of correlation. As a result of both, the position of the maximal crosscorrelation was not always at the center of the node.

To illustrate the point we have included an example of automatic node tracking in figure $4.7 \mathrm{a}$. As the error introduced by the loss of the position of the node center is amplified over time, we found this method to be unsuitable for reliable node tracking regarding our cells. Therefore, a script has been developed which served the purpose of manual node tracking. With this aim in mind, the images were upscaled by a factor of 5 through bicubic extrapolation and the center of the node was then marked manually in the magnified image. To compare the performance of both approaches we display the respective results of node tracking for an example network node in figure $4.7 \mathrm{~b}$ and c. As evidenced by the plots, the automatic tracking leads to node displacements between images which are too short. As mentioned before, this is mainly due to morphological changes in the images and to motion out of the focal plain. The reason why morphological changes in particular lead to systematically smaller node trajectories lies in the fact that the correlation function finds not only the best correlation for the node itself, but also for the fluctuating bundles branching out of the node. The larger the correlation window, the larger the influence of the node environment and hence the smaller the detected trajectory is. To reduce the influence of the bundles on the correlation we also considered further reducing the size of the correlation window. However, for smaller correlationwindows, the variations in intensity, due e.g. to motion in $z$, become more important for the tracking and thus lead to larger errors when determining the nodes center. Consequently, we followed the manual approach to determine the trajectories of the nodes.

The thus obtained node trajectories needed to be corrected for drift or overall network motion. We consequently utilized the information which we gained from the calculation of the image-to-image cross-correlation (see section 4.2.2 to this end, since the spatial correlation between two image frames $I_{t}$ and $I_{t+\tau}$ (equation (4.14)) contains the required information. In essence, the coordinates $x^{\prime}$ and $y^{\prime}$, for which this correlation is maximal, reveal the average displacement of the whole network. Therefore, we corrected the extracted node trajectories with the corresponding shifts of the network detected by correlation function.

To characterize the different node trajectories we determined the radius of gyration for each node, as well as the mean-square-displacement. A brief description of the two parameters follows subsequently. 


\section{Radius of Gyration}

The radius of gyration $R_{\mathrm{g}}$ describes the spreading of an ensemble of $N$ points. That is, the average distance between the positions $\overrightarrow{r_{k}}$ and the centroid of all points $\langle\vec{r}\rangle$ is calculated:

$$
R_{\mathrm{g}}^{2}=\frac{1}{N} \sum_{k=1}^{N}\left(\overrightarrow{r_{k}}-\langle\vec{r}\rangle\right)^{2} .
$$

If the points $\overrightarrow{r_{k}}$ represent positions along a trajectory, $R_{\mathrm{g}}$ reflects the typical size of the space covered by e.g. a KIF network node following the previously mentioned trajectory.

\section{Mean-Square-Displacement}

In addition to the determination of $R_{\mathrm{g}}$, we also determined the mean-squaredisplacement (MSD) of the node positions over time. Contrary to $R_{\mathrm{g}}$, which contains only spatial information about a node trajectory, the MSD reveals the temporal evolution of the node's movement:

$$
\left\langle(\vec{r}(t+\tau)-\vec{r}(t))^{2}\right\rangle_{t}=\left\langle\Delta r^{2}\right\rangle_{t}=2 n \cdot D \cdot \tau^{\beta}
$$

Here, $n$ represents the number of dimensions in space, \langle\rangle$_{t}$ indicates an average over all recorded images, $\tau$ is the time shift between two images and the exponent $\beta$ characterizes the type of motion: a subdiffusive $(\beta<1)$, a superdiffusive $(\beta>$ 1 ) or a diffusive behavior $(\beta=1)$. Whereas the diffusive behavior refers to the random Brownian movement observed for thermally driven particles, subdiffusion and superdiffusion describe motion that is decelerated or accelerated in comparison to diffusion, respectively. The deviation from thermal diffusion can, for example, be caused by passive constraining elements such as neighboring cytoskeletal components (e.g. [98]), or active elements like motor proteins which enhance movement (e.g. 99]). As can be seen from the equation above, an average velocity
$\sqrt{\left\langle(\vec{r}(t+\tau)-\vec{r}(t))^{2}\right\rangle_{t}} / \tau$ can be expressed in terms of $D$ - the diffusion coefficient. Hence, $D$ can be utilized to characterize the motility of, for example, our KIF network nodes. However, it is important to note that for motion other than a Brownian one, the parameter $D$ needs to be understood as an effective rather than an actual diffusion coefficient, that is $D$ turns into $D_{\text {eff. }}$.

Equation (4.19) above is written in a generalized form and applies to motions in any dimensions in space. In our case, we assume the motion of the nodes to be constrained to $2 \mathrm{D}$ because of the restrictions imposed by the keratin-free nucleus 
on top and the cell membrane at the bottom of the KIF network (see section 4.2). Hence, we obtain for the MSD of the nodes the 2D equation

$$
\left\langle\Delta r^{2}\right\rangle_{t}=4 D_{\mathrm{eff}} \cdot \tau^{\beta}
$$

Here, we assume that the properties of the node behavior, i.e. $D_{\text {eff }}$ and $\beta$ are constant over the whole time period. Nodes which change their morphology, e.g. due to a 'zipping' of multiple bundles, thus have to be excluded from our investigations.

When plotted logarithmically, the effective diffusion constant $D_{\text {eff }}$ shows up as the intercept and the exponent $\beta$ as the slope of the resulting linear function:

$$
\log \left(\left\langle\Delta r^{2}\right\rangle_{t}\right)=\log \left(4 D_{\text {eff }}\right)+\beta \cdot \log (\tau)
$$

\subsection{Reduced Keratin Network Motion under Flow}

In the following, we present our results for the characterization of the keratin network motion in the presence or absence of external shear stress. As mentioned previously (see section 4.2), we focused our analysis on the KIF network region below the nucleus, thus taking advantage of the fact that for this region the network motion was mainly restricted to $2 \mathrm{D}$, the network morphology altered only little over time and imaging conditions were good.

First, we acquired images of cells in microfluidic channels without flow. These data served as a reference for the comparison with the network response to shear stress. For the analysis of the KIF network under shear forces, the first 5 min were excluded. The initial time lapse was introduced to allow the cells to adjust to the new conditions. Yoon et al. have revealed that bundles of keratin are singularly and collectively displaced within 2-4 min after the establishment of shear stress in the direction of flow while a displacement of the cell in its entirety was not detected [51. With the exclusion of the first 5 min of data, we therefore ensure that the determined characteristics solely reflect the shear-induced changes in KIF motion rather than the onetime dislocation of the bundles upon initiation of fluid flow.

\subsubsection{Global Approach: 2D-Cross-Correlation}

The image-to-image cross-correlation yields a parameter which describes the average similarity between two images that are a time span $\tau$ apart. Figure 4.8 shows an example of typical CC curves plotted against $\tau$ for a cell in the absence and 


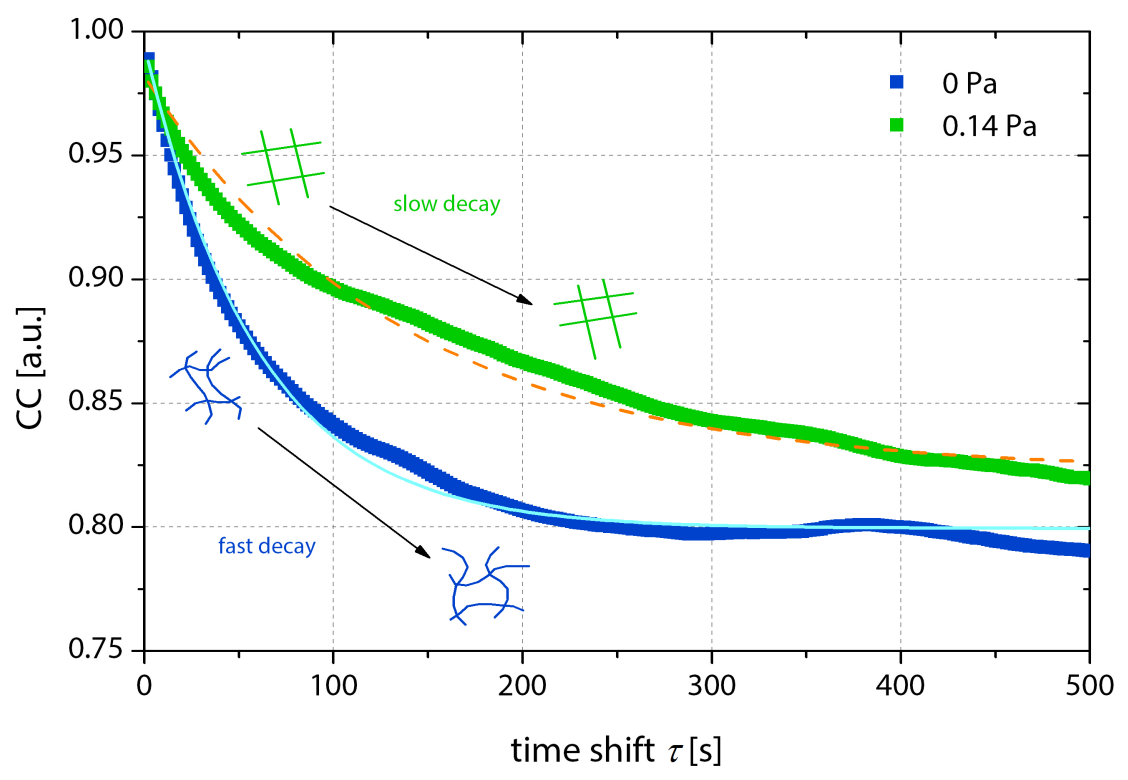

Figure 4.8: Example of image-to-image cross-correlations for microscopy images of a single cell both without shear flow (blue data points) and under shear conditions (green data points). The data are fitted with an exponential function (cyan solid line for no-flow and dashed orange line for flow conditions). Under the influence of shear forces the decay time becomes larger, signifying that the network is more persistent. In this example, the characteristic decay time is $184 \pm 4 \mathrm{~s}$ for shear and $69 \pm 1 \mathrm{~s}$ for no-shear conditions.

presence of shear stress, respectively. The curves in the plot show a decay which is analyzed by fitting an exponential function to the data. The fits give access to characteristic decay times over which image-to-image similarity is lost, which in turn reflects typical time scales for the network motion. All CC curves of the 12 cells are depicted in figure 4.9 and an overview of the corresponding decay times extracted for these cells is given in figure 4.10a. To underline the effect of shear stress on the KIF network dynamics we have, moreover, calculated the difference between the decay times under shear and no-shear conditions for each individual cell in the aforementioned ensemble and plotted it in form of a histogram in figure 4.10 b. We find that a majority of 10 out of 12 cells show longer time scales for the KIF network dynamics when shear stress is applied to the cells. On average, we identify a decay time of about $160 \pm 15 \mathrm{~s}$ for non-sheared cells and a decay time of about $210 \pm 15 \mathrm{~s}$ for the same cells under shear conditions (standard errors of mean). Thus, the network remains similar over a longer period in time under shear conditions as compared to the situation without the influence of external forces. Consequently, the motion of the bundles is less pronounced when shear flow is applied to the cells. 


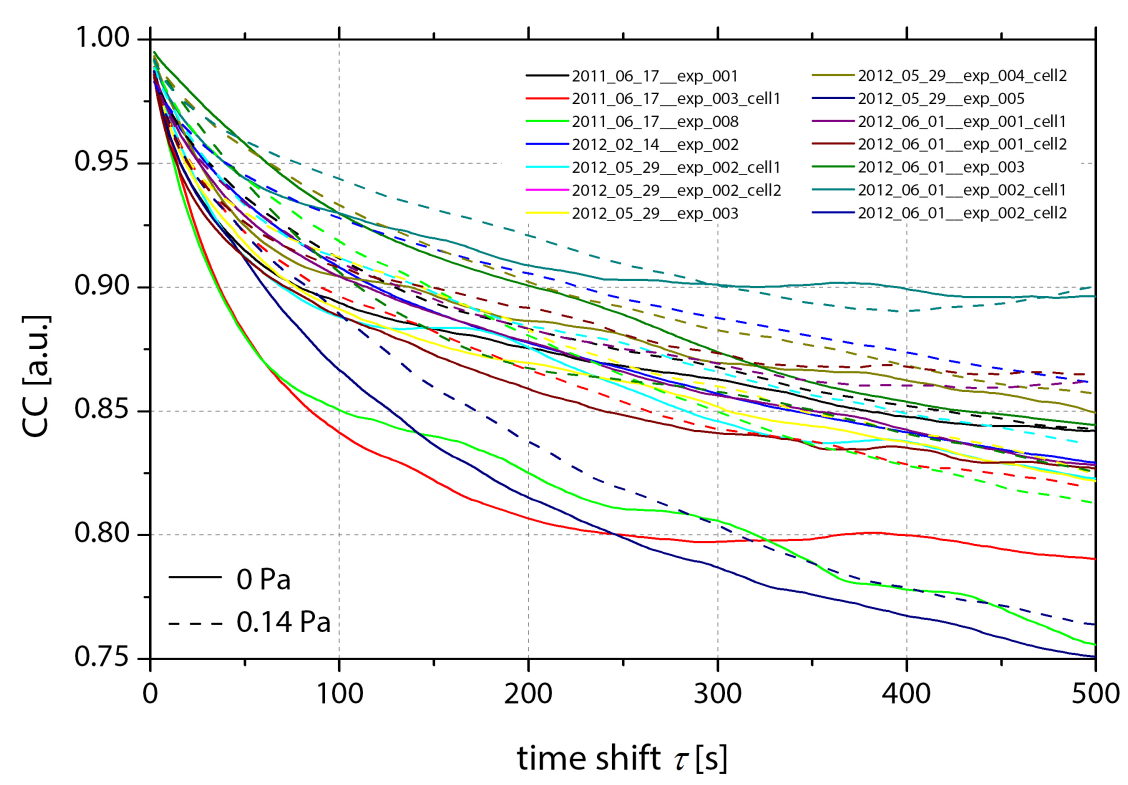

Figure 4.9: Overview over all image-to-image cross-correlations of 12 cells (solid lines represent no-shear conditions and dashed lines show data of the same cells under the influence of shear). For 10 cells, the application of shear stress leads to a slower decay of the correlation function as compared to the situation in the absence of external forces. On average, we found characteristic time scales of $160 \pm 15 \mathrm{~s}$ for non-sheared cells and a decay time of about $210 \pm 15 \mathrm{~s}$ for the cells when shear forces are applied (standard errors of mean).

\subsubsection{Local Effects of Shear Flow on Keratin Networks}

Apart from analyzing the image-to-image CC, which enables the simultaneous investigation of the entire KIF network under the nucleus, we also pursued a more local approach to complement the global one. To this end, trajectories of 38 KIF network nodes in 10 cells were separately investigated under shear and no-shear conditions. Nodes were selected which were observable for the whole data set including shear and no-shear conditions to ensure good comparability. Consequently, the signals of the nodes had to be not overlaid by signals of other nodes or bundles and the morphology of the node had to be sufficiently stable to be able to deduce its center over the whole time period. Typical trajectories of single nodes, which were thus obtained, resemble the example shown in figure 4.11. For each trajectory and shear condition we then extracted a radius of gyration and plotted the temporal evolution of the mean-square-displacement (MSD). As described in more detail later on, the MSDs were evaluated to obtain an effective diffusion constant for each node trajectory. Both the radius of gyration and this effective diffusion constant were finally averaged over all nodes in a single cell in order to characterize the overall motion of 
(a)

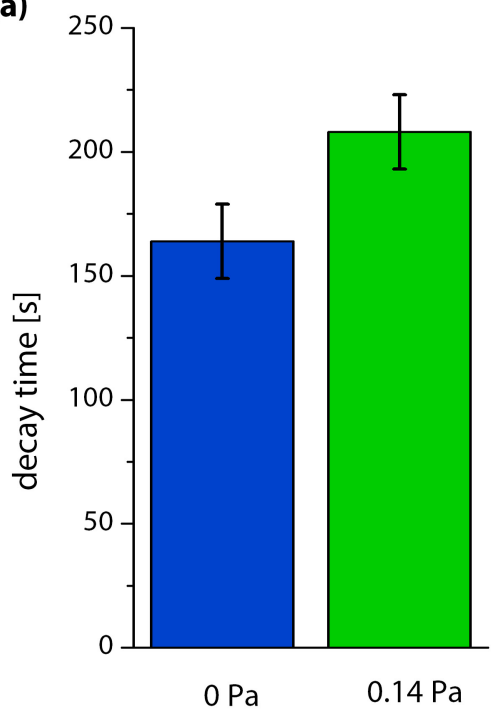

(b)

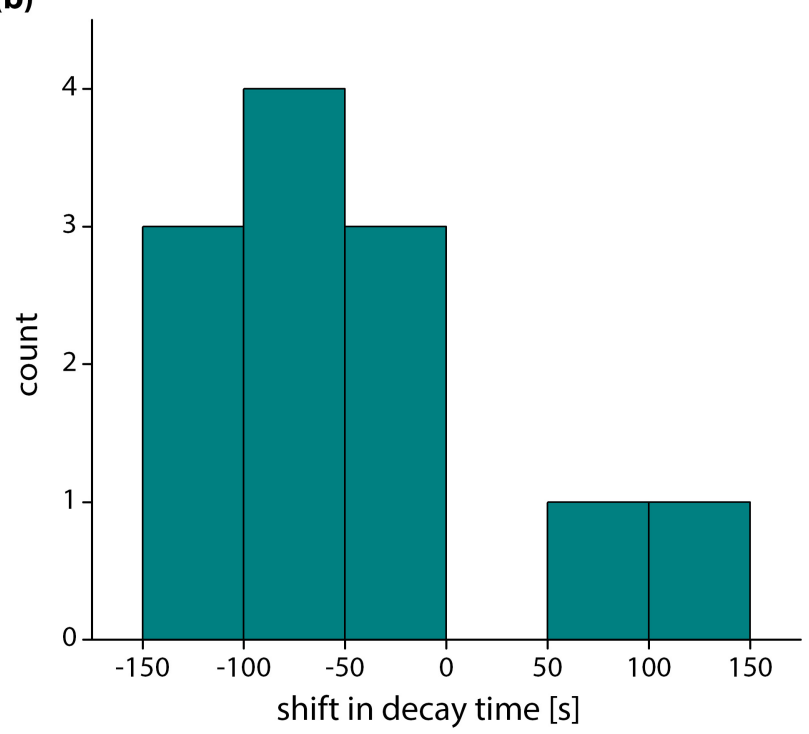

Figure 4.10: (a) Averaged over the cell ensemble, the characteristic time scale $t_{\text {char }}$ increases by about $20 \%$ for sheared cells (error bars indicate the standard error of mean). (b) Histogram of the differences in the characteristic time scales of KIF network dynamics between the non-sheared and sheared state of individual cells. In total, the network remains similar over a longer time period for this mechanical state of the cells (data based on 12 cells, $\Delta t_{\text {char,no-shear }}-\Delta t_{\text {char,shear }}$ ).

network nodes in the presence and absence of external shear. An overview of trajectories which belong to complete datasets of two cells is attached in appendix A. In addition to the node trajectories, the appendix further contains the corresponding values of $R_{\mathrm{g}}$ and the MSD.

\section{Radius of Gyration}

As mentioned in section 4.1.2 we were careful to setup comparable experimental conditions for all cells used for the analysis. Assuming the experimental outcome to be representative for the cell population instead of reflecting cell-to-cell differences, we therefore summarized our results on the radius of gyration $R_{\mathrm{g}}$ first for every cell. To this end, the arithmetic mean of all nodes which belong to one cell was calculated together with the standard error of mean $\sigma_{\text {err }}$. In total, data of 38 nodes in 10 cells have been taken into account (every cell contain 3 to 5 nodes which have been analyzed). Afterwards, we calculated the weighted mean of $R_{\mathrm{g}}$ (see equation (4.22), where $i$ is an index counting the cells) with the corresponding variance of weighted mean $\sigma_{\mathrm{err},\left\langle R_{\mathrm{g}}\right\rangle}$ (see equation 4.23) ) to obtain one value for the whole cell population: 


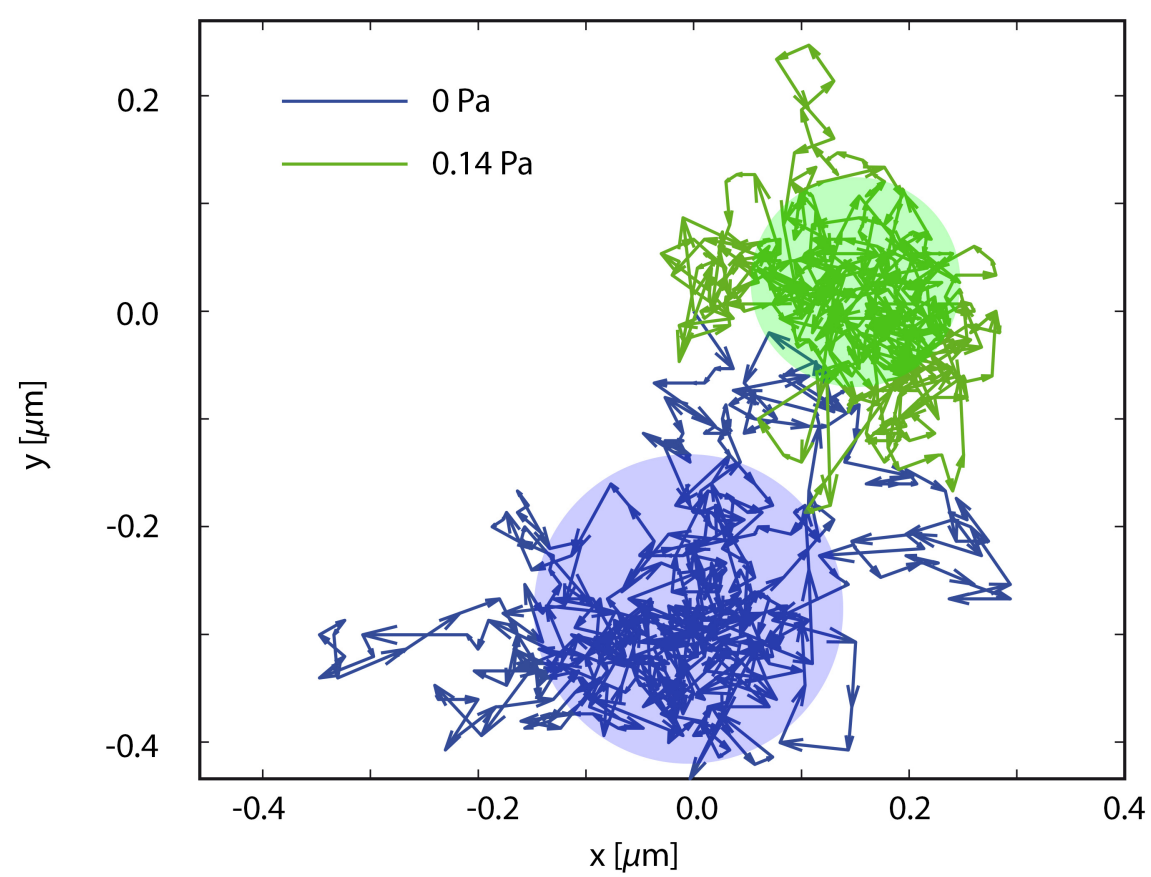

Figure 4.11: The lines depict the trajectories of a node in a keratin network for the two situations, without external forces (blue) and upon application of shear stress (green). The trajectories are corrected for the cell centroid motion. When shear forces are established, the motility of the nodes is visibly reduced as seen by the smaller spreading of the node trajectory. To better visualize trajectory spreading we have furthermore included circles with sizes corresponding to the radii of gyration of the trajectories (equation 4.18); centers approximately aligned with the centroids of the nodes' motion).

$$
\begin{aligned}
\left\langle R_{\mathrm{g}}\right\rangle & =\frac{\sum_{i} \frac{R_{\mathrm{g}, i}}{\sigma_{\mathrm{err}, i}^{2}}}{\sum_{i} \frac{1}{\sigma_{\mathrm{err}, i}^{2}}} \\
\sigma_{\mathrm{err},\left\langle R_{\mathrm{g}}\right\rangle} & =\frac{1}{\sqrt{\sum_{i} \frac{1}{\sigma_{\mathrm{err}, i}^{2}}}} .
\end{aligned}
$$

The calculations were performed for no-shear and shear conditions separately. As depicted in figure 4.12 we find that $R_{\mathrm{g}}$ decreases when external shear stress is applied: The initial value of $0.18 \pm 0.01 \mu \mathrm{m}$ for non-sheared cells is lowered to $0.13 \pm 0.01 \mu \mathrm{m}$ when external forces are applied to the cells. The effect is also clearly visible, if we look at the shift in $R_{\mathrm{g}}$ caused by shear stress for the individual cells. An overview over the results is shown in a histogram in figure $4.12 \mathrm{~b}$, where the difference between the radii is depicted $\left(R_{\mathrm{g}, \text { no-shear }}-R_{\mathrm{g}, \text { shear }}\right)$. We found for 9 out of 10 cells that $R_{\mathrm{g}}$ is reduced upon the application of shear forces. 
(a)

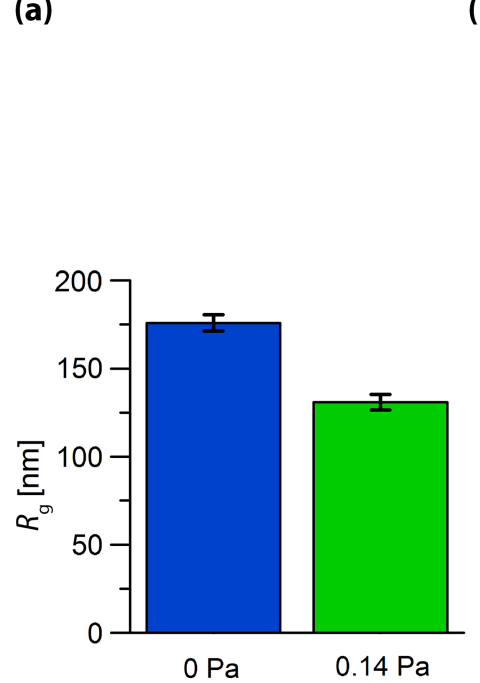

(b)

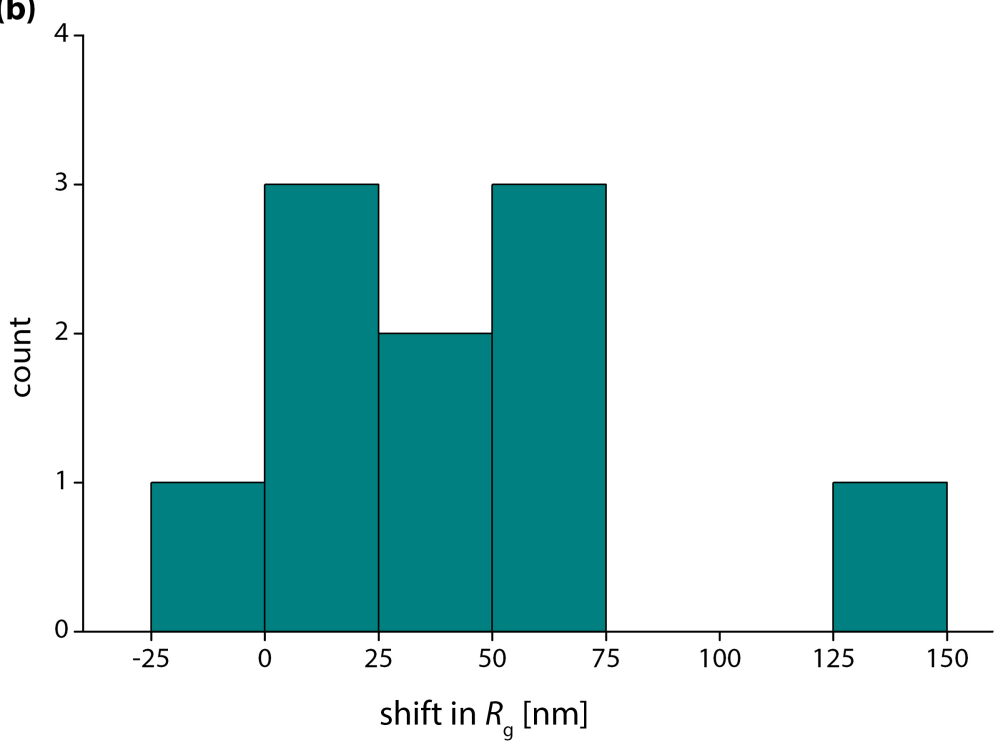

Figure 4.12: (a) Averaged over the cell population (weighted mean), the radius of gyration decreases by about $40 \%$ for KIF networks in cells when shear stress is applied (error bars indicate the variance of weighted mean). Accordingly, the spreading of the trajectories is reduced. (b) Histogram of the differences in the radius of gyration for individual cells in a comparison between shear and no-shear conditions (data based on 38 nodes in 10 cells, $R_{\mathrm{g}, \text { no-shear }}-R_{\mathrm{g}, \text { shear }}$ ).

\section{Mean-Square-Displacement}

Applying equation 4.19 to our data, we obtained MSD curves for each node in a cell both in the presence and absence of shear stress. An example for an MSD curve is depicted in figure 4.13. Here, however, we have only in this case averaged the MSDs over all nodes in a single cell to better illustrate the typical behavior of network node motion in a cell. As evidenced by the plot, there is a regime toward low time shifts, for which the MSD shows the linear behavior expected from theory (equation (4.21)). Since the statistics become worse for larger time shifts we focus on this linear regime for the following analysis.

By fitting linear functions to similarly linear segments in all our data sets, we analyzed the intercept of the MSDs for each node trajectory and deduced the effective diffusion coefficient according to equation 4.21. The obtained values are then averaged for all nodes in a single cell and the corresponding standard error of mean was calculated. For the example cell shown in figure 4.13 , we already find by optical inspection of the plot that the intercept for the green curve with shear stress is lower than the one for the blue curve relating to the absence of shear. Consequently, the effective diffusion coefficient for the KIF network in the cell decreases under the influ- 
ence of micro-flow induced shear. This trend is confirmed throughout the population of analyzed cells, as indicated by the histogram in figure $4.14 \mathrm{~b}$ showing the shift in $D_{\text {eff }}$ caused by shear stress application to the individual cells. For all 10 cells, $D_{\text {eff }}$ is reduced when the cells are exposed to external shear forces. When determining the weighted average over the whole cell population in analogy to equation 4.22, we find a shift of the effective diffusion constant from $(1.57 \pm 0.01) \cdot 10^{-16} \mathrm{~m}^{2} / \mathrm{s}$ (no-shear) to $(0.83 \pm 0.01) \cdot 10^{-16} \mathrm{~m}^{2} / \mathrm{s}$ under shear conditions (weighted errors of mean; see figure 4.14 a).

The slope of the linear regimes in the data is on average below 1 indicating a subdiffusive behavior $(0.84 \pm 0.01$ for no-shear and $0.89 \pm 0.01$ for shear conditions when calculating the weighted average and variance of weighted mean of the cell population). This is, on the one hand, counter-intuitive when taking into account that the slope ( $\beta$, see section 4.2.3) indicates the type of diffusion, where an active driven motion generally leads to a super-diffusion behavior $(\beta>1)$. On the other hand, we have to consider that the observed nodes are not able to move freely but are constrained in their moveability due to their nature of being part of a network.

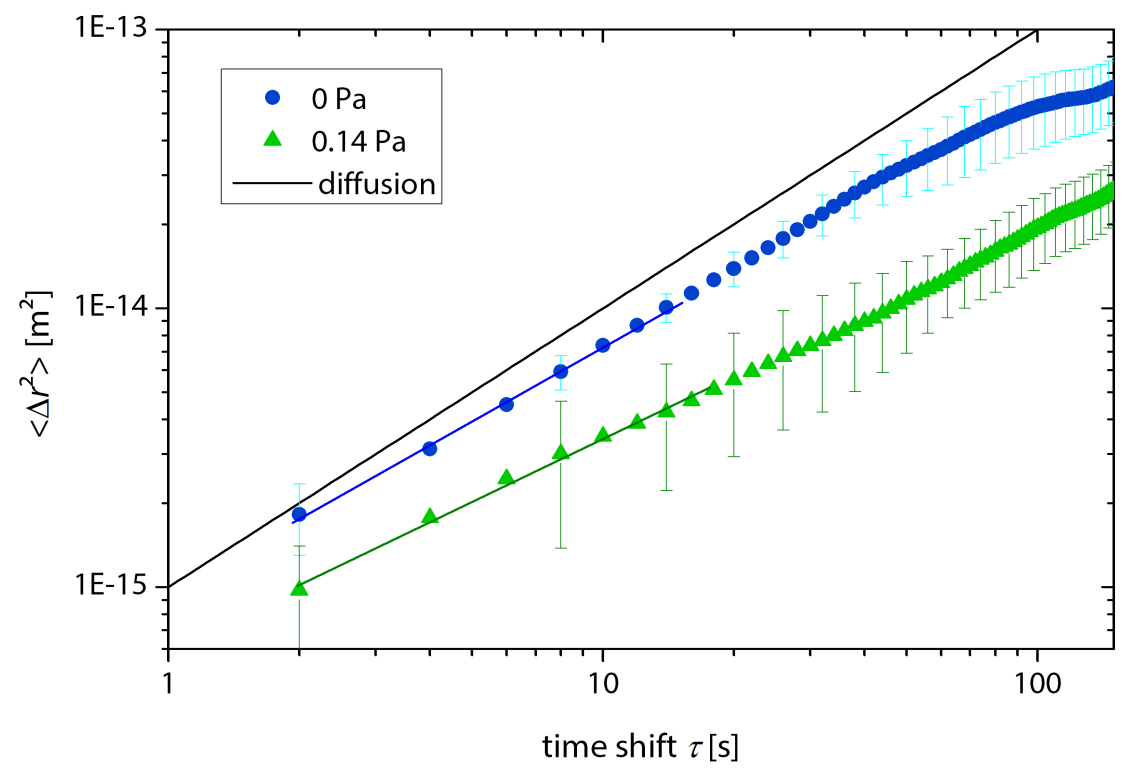

Figure 4.13: Example of the mean-square-displacement for the KIF network nodes in one cell both in the absence (blue dots) and presence of shear forces (green triangles). Each data point represents the average over 5 nodes and the error bars indicate the standard error of mean. In the linear regime of the curves we fitted a linear function to the data and extracted the intercept to obtain an effective diffusion constant $D_{\text {eff. For comparison }}$ purpose, we include an example for the MSD according to diffusive motion with a slope of 1. 
(a)

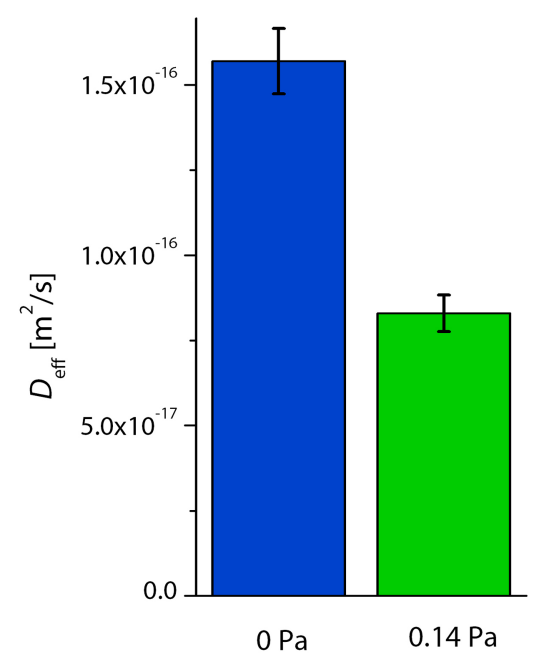

(b)

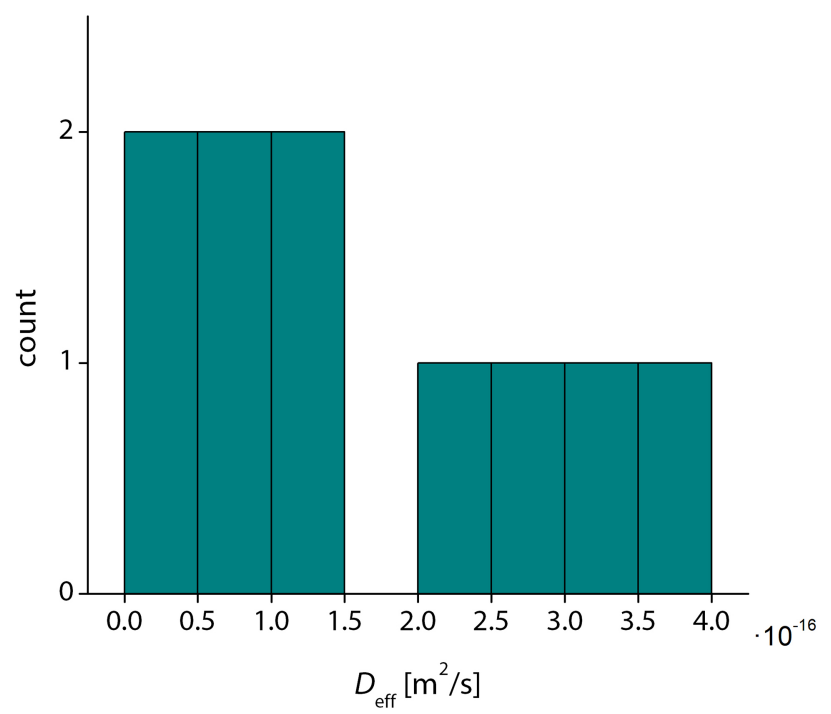

Figure 4.14: (a) Calculating the weighted average for the cell population (10 cells in total), we find a reduction in $D_{\text {eff }}$ of $47 \%$ when shear forces are applied to the cells. (b) Histogram of the differences in the average effective diffusion coefficient $D_{\text {eff }}$ for the KIF network nodes in single cells in a comparison between their non-sheared and sheared state (data based on 38 nodes in 10 cells, $D_{\text {eff,no-shear }}-D_{\text {eff,shear }}$ ).

\subsection{Treatment with Blebbistatin}

To shed light on the question about the origin of the KIF bundle motion in cells and whether the observed effects of shear forces on the network dynamics are caused by active responses of the cell or by passive material properties, we investigated the influence of motor protein activity on the KIF network motion. Since no IF associated motor proteins are known, we hypothesized other motor proteins to be involved. Likely candidates we considered are actin-associated myosin motors, which are known to generate forces on a cellular level and thus fulfill both static and dynamic functions in the cells [100, chapter 18]. In detail, myosin II provides inner tension to support the cell shape, for example during cell adhesion. Also, myosin I and V drive transport of some vesicles in the cell while myosin II is responsible for movements like muscle cell contraction and the pinching off of dividing cells during mitosis. Because of its role as a contractile force generator we focused in particular on myosin II. To evaluate the influence of myosin on the observed bundle motion, cells were therefore treated with the myosin-II-inhibiting drug blebbistatin (SigmaAldrich) 101, 102. For reference purposes, we first observed the SK8/18-2 cells in drug-free cell culture medium in a petri dish over a time span of $2 \mathrm{~min}$ and in time intervals of $2 \mathrm{~s}$ using epifluorescence microscopy. After removing the medium from 
(a)

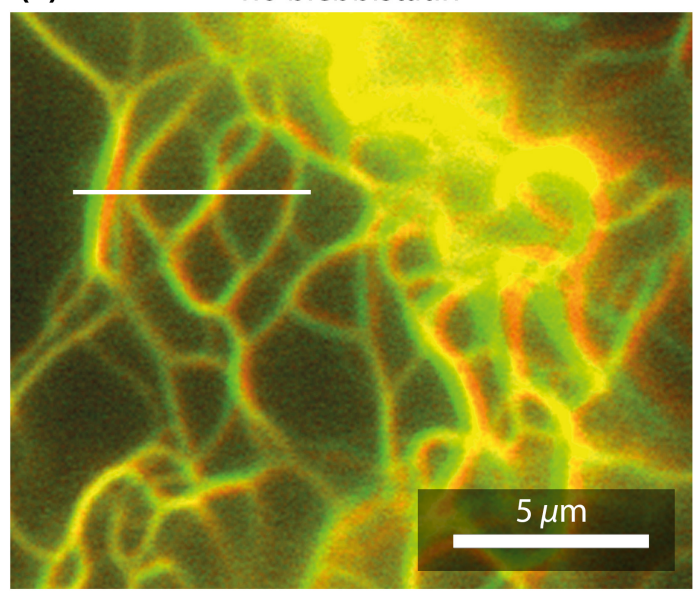

(c)

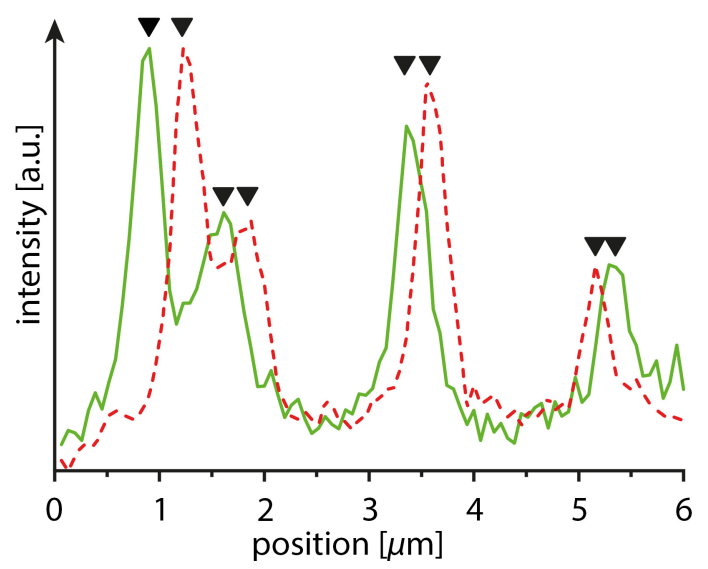

(b)

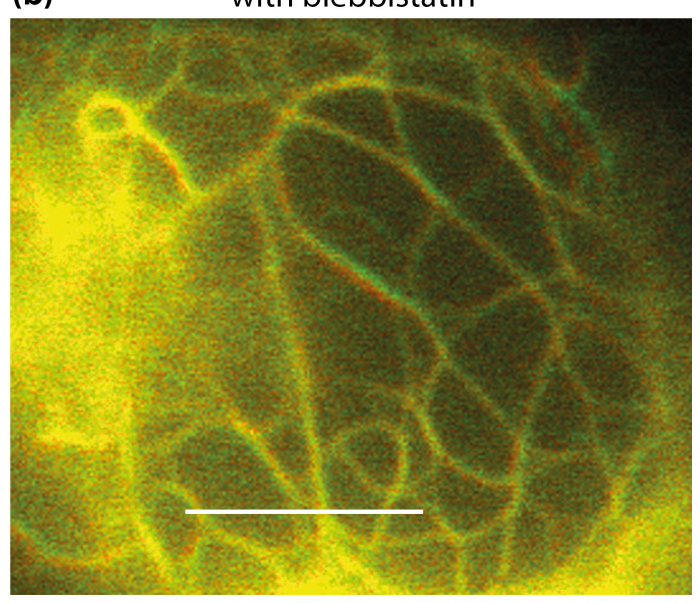

(d)

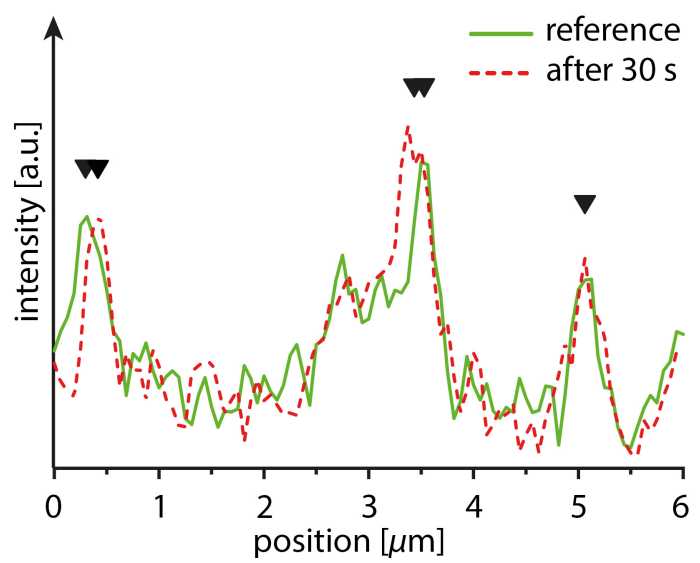

Figure 4.15: (a), (b) Overlays, each of two fluorescence microscopy images separated by a time shift of $30 \mathrm{~s}$, for the respective situations before and after the treatment of the cell with the myosin-II-inhibiting drug blebbistatin. The fluorescence images are color coded in green and red according to their chronological order to visualize the bundle motion between the two frames. In addition, the bundle motion is illustrated by intensity profiles $((\mathbf{c}),(\mathbf{d}))$ taken along the white lines in the image overlays and displaying the same color code as before (green line for the first image frame and red dashed line for the frame taken after a delay of $30 \mathrm{~s})$. Peak positions in the intensity profiles are furthermore highlighted with black arrow heads. While there is distinct motion in an untreated cell $((\mathbf{a}),(\mathbf{c}))$, the bundle motility is noticeably reduced under the influence of blebbistatin $((\mathbf{b}),(\mathbf{d}))$. 
the petri dish, the cells were exposed to $1 \mathrm{ml}$ of a solution of $100 \mu \mathrm{M}$ blebbistatin in medium for a time period of $4 \mathrm{~h}$. Afterwards, the cells were again observed over $2 \mathrm{~min}$ in $2 \mathrm{~s}$ intervals.

To visualize the KIF bundle motion before and after treatment of the cells with blebbistatin, we extracted from the recorded data two fluorescence images for each experimental condition respectively with a time shift of $30 \mathrm{~s}$ between the frames. Both overlays are depicted in figure 4.15 in addition with intensity profiles which are taken along selected horizontal lines in the images. The intensity profiles are included to quantify the bundle displacements observed in the overlays. As seen in the figure, the movement of bundles in an untreated cell is clearly visible with final displacements of up to $0.5 \mu \mathrm{m}$ after $30 \mathrm{~s}$. After the treatment with blebbistatin the bundle displacements are perceptively reduced. Here, the motion is in the range of about $0.1 \mu \mathrm{m}$ within the same time span of $30 \mathrm{~s}$.

We abstain from a full characterization of the remaining motion of the keratin network because we are limited in the number of images that we are able to take of cells under the influence of blebbistatin. The reason for this lies in the fact that blebbistatin is degraded under illumination with UV and blue light. As revealed by Kolega, the photochemical process involved is toxic in cells and can even result in cell death $[103$. Since our keratin network is observed with UV/blue wavelengths, we are only able to record a small number of images $(\sim 50)$ before the cell begins to contract visibly.

\subsection{Discussion}

In the previous section 4.3, the dynamics of KIF bundles in living SK8/18-2 cells were investigated in a comparison between two experimental scenarios. In the first scenario, external forces were non-existent, while in the second scenario we applied shear stress to the cells using microfluidic methods. By calculating image-to-image cross-correlations, we were able to access shear-stress-induced changes in network motion for the entire cellular region under the nucleus. This method was complemented by an investigation of the trajectories of individual, local network nodes for each scenario. Here, the trajectories were characterized by determining the radius of gyration and the mean-square-displacement (MSD). Both the analysis of the radius of gyration as well as the study of the MSD support the result already obtained with the global approach: We find a distinct reduction in keratin bundle motion when the cells are exposed to shear stress.

In the following, we discuss two possible explanations for the observed effect of 
shear stress on KIF bundle displacement: First, we consider the reduced bundle motion to result from changes in the passive mechanical properties of the keratin network itself. Secondly, we contemplate the possibility that active processes in the cell govern KIF bundle dynamics and that hence a shear-induced rise in motor activity, leading to a higher effective rigidity of the cell, accounts for the reduced bundle motion.

There are several indications for the first argument. In vitro studies using rheology measurements have revealed that for keratin gels the stress increases more than linearly for large deformations 19,42 . This strain hardening behavior is explained by Storm et al. with entropic effects 41. That is, stretching a flexible filament pulls out fluctuations and reduces the number of possible conformations. As a result, the entropy of the system is reduced which is opposed by a restoring force. The filament can therefore be thought of as an entropic spring.

For illustration purposes, we describe the idea of an entropic spring for the example of an ideal or freely joined chain (for an in-detail description, see [104, p. 78]), where a polymer is made up of $N$ links, each of which has a length $r$. The links have random orientations and consequently the shape of the polymer is described by a random walk. While the total unfolded contour length of the polymer is described by $L=N r$, the end-to-end distance is described by $\vec{R}=\sum_{i} \vec{r}_{i}$. The distribution of possible end-to-end distances for large $N$ is expressed as a Gaussian function

$$
P(\vec{R}, N)=\left(\frac{3}{2 \pi \cdot N r^{2}}\right)^{3 / 2} \exp \left(-\frac{3 \vec{R}^{2}}{2 N r^{2}}\right) .
$$

With the entropy given by $S=k_{\mathrm{B}} \ln (P)+S_{0}$ and the free energy described by $F=U-T S$, we obtain the energy equation

$$
F(\vec{R}, N)=F_{0}+\frac{3 k_{\mathrm{B}} T}{2 N r^{2}} \overrightarrow{R^{2}}
$$

Thus, a reduction of possible configurations, e.g. a stretching of the polymer (lower $S$ ), will increase the energy $F$ by a term $\propto \vec{R}^{2}$.

The obtained strain stiffening effects are only relevant for large deformations of more than $\sim 50 \%$ of stretching for keratin 42 . Since the keratin network in our experiments was not directly exposed to forces, the resulting deformation was not that pronounced. Indeed, our cells were solely sheared with a stress up to $0.27 \mathrm{~Pa}$ (see section 4.1.2 and the resulting deformation of the cell itself was too low to affect the keratin network within in such a way as to initiate strain hardening. Further support of the point that the deformation of the cell and thus also of the network is neglectable is provided by the fact that we do not detect any direct morphological 
changes of the network in our microscopy images. Also, we focused our analysis on the central network region just below the nucleus, which should be less affected by cell deformations than at the periphery.

However, previous studies, which are discussed in the following, have shown that keratin networks in cells do stiffen when the cells are exposed to shear stress. In contrast to passive mechanical properties of the keratin, these changes are attributed to active responses of the cells to the external forces. The resulting network stiffening is thus a consequence of an active reorganization of both the keratin bundles and the network morphology. Sivaramakrishnan et al., for example, observed a reorganization of the KIF network within $4 \mathrm{~h}$ of shear stress application (1.5 Pa), resulting in more homogeneous mesh sizes across the cell. This, in turn, leads to an increase in the mean elastic modulus of the cell of $40 \%$ 71. Furthermore, they have found that in cells, which were exposed to a shear stress of $3 \mathrm{~Pa}$ for a time period of $1 \mathrm{~h}$, the keratin bundles are thicker and the network is reinforced [72]. Using FRAP measurements, they have revealed that this reinforcement is caused by a twofold increase in the subunit exchange rate in KIFs. Moreover, the emergence of thicker bundles after shear stress application ( 0.7 or $1.5 \mathrm{~Pa}$ for time periods up to $10 \mathrm{~h}$ ) has also been observed by Flitney et al. [38]. Further supporting their observation, the authors have identified a loss in most free keratin particles in the cytosol under the influence of shear stress. They conclude that these particles are integrated into the existing network - a process which is accompanied by an increase of keratin phosphorylation. When cells are exposed to shear stress for an even longer time period, like $24 \mathrm{~h}$, the keratin network becomes disorganized and begins to disassemble [69,70]. An overview of the temporal progress of the cellular response to shear stress with regard to keratin is shown in figure 4.16 .

The reorganization and reinforcement of the cellular KIF network in the presence of shear stress corroborates the idea that one of the main functions of keratin is to support the cell, ensure its integrity under the influence of external forces and to represent a mechanical buffer system (see chapter 2). This role becomes most evident when considering that blocking the shear stress-mediated reorganization of the KIF network by inactivation of the involved kinases leads to a reduced viability of the cells [72]. For our study, these long term changes in the KIF network are less relevant since we focus on short-termed (5-20 min) responses of the cell to shear stress. Furthermore, we analyze a region below the nucleus, where the changes in network structure are less pronounced [71]. Because of our selection of a stable network region at the cell center and because we want to address the question whether there is an immediate to minutes-consuming response of the cell which helps 


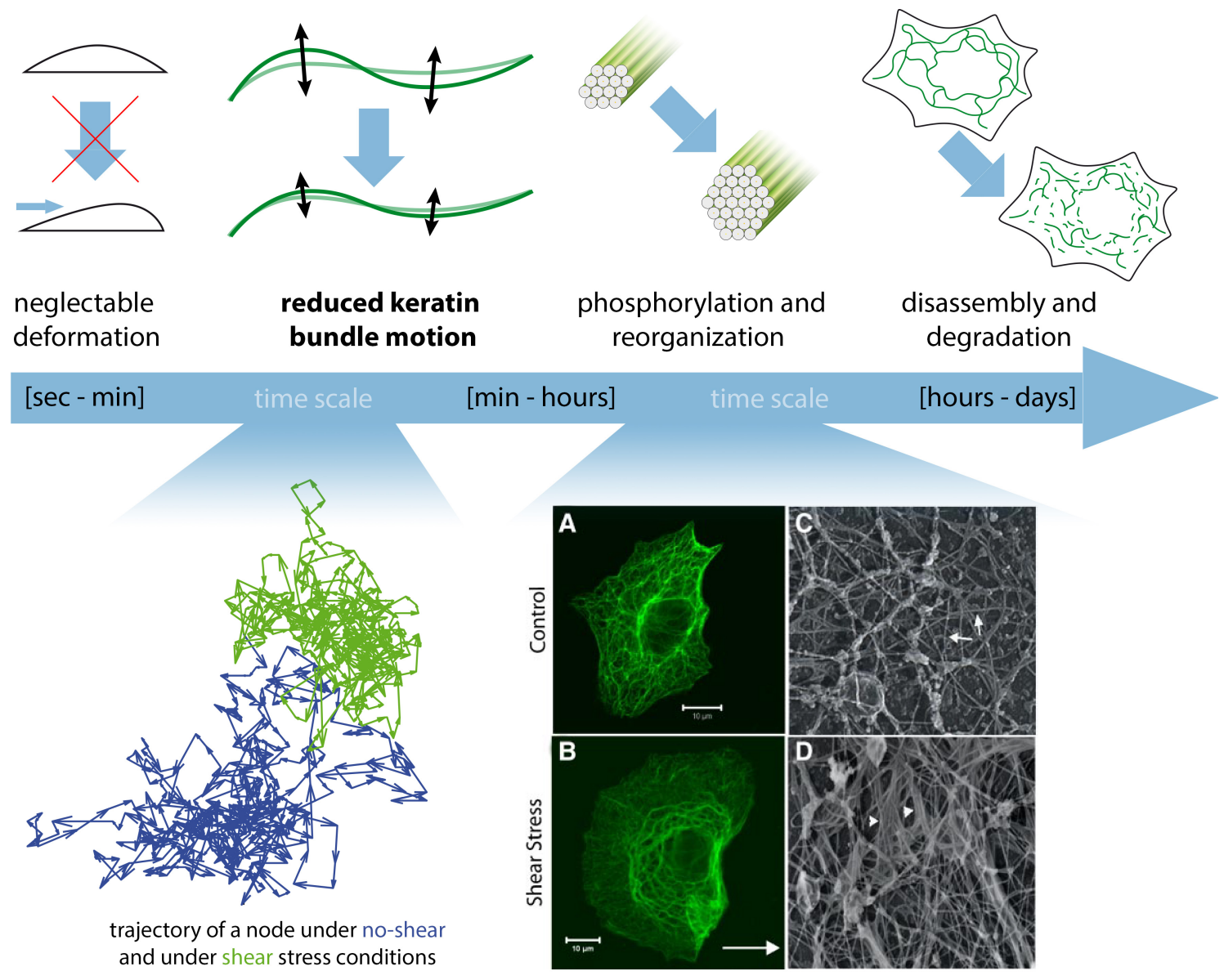

Figure 4.16: There are different responses of the cell to external shear forces, which take place on different time scales. As an immediate and passive reaction, the cell deforms due to the external force. However, the deformation is very small and thus neglectable (in the order of magnitude of $10 \mathrm{~nm}[96]$ ). In the range of minutes, we have identified a reduction in the bundle and keratin network node motion. On longer time scales, phosphorylation of the KIF network leads to larger bundles, the formation of tonofibrils and a reorganization of the network (in-detail description given in the text). After the application of shear stress to cells over at least $24 \mathrm{~h}$, the KIF network disassembles and degrades. Image in the bottom right taken from reference $\overline{\mid 72}]$.

to withstand the external mechanical forces, we can neglect network reorganization.

A short-term response of the cell to external shear stress may be realized by an indirect mechanism like an altered motor protein activity. A simple estimation provides evidence that the observed bundle motion in cells is not Brownian but a result of an active process i.e. it is motor protein driven. The results of the MSD analysis reveal that the maximum squared distance a KIF network node moves is of the order of $10^{-13} \mathrm{~m}^{2}$. By multiplying this area with a typical mesh size of the KIF network, which is of the order of $1 \mu \mathrm{m}$, we obtain a volume scale of $10^{-19} \mathrm{~m}^{3}$. If we 
assumed the motion of the keratin network to be Brownian and purely thermal, we would estimate the elastic modulus of the network by dividing the thermal energy $k_{\mathrm{B}} T \approx 4 \cdot 10^{-21} \mathrm{~J}$ by the previously calculated volume scale. We would thus arrive at a modulus of $0.04 \mathrm{~Pa}$, which is extremely small. Walter et al. have shown that the elastic modulus of an isolated, epithelial keratin network is about $10 \mathrm{~Pa}$ [105]. This estimate clearly shows that the observed bundle motion is not Brownian but arises from active processes.

The idea of an altered motor protein activity would be supported by an increase in calcium ion concentration. In fact, calcium is crucial for the regulation of myosin II motor proteins since it activates the myosin light-chain kinase (MLCK). MLCK regulates the myosin light-chain (MLC) phosphorylation, which is needed for myosin II activity [106]. We propose that in shear stressed cells an increased calcium concentration causes an increase in myosin motor protein activity. To test whether a rise in calcium ion concentration is indeed present in our cells under shear stress, we performed calcium imaging. Here, ratiometric imaging has become a powerful tool to investigate changes in ion concentrations. This method is based on indicators which have different excitation or emission wavelengths depending on the local ion concentration. By dividing the signals of both wavelength regimes, it is possible to get information about the ion concentration. For our purposes, we used the indicator Indo-1 (Invitrogen, Darmstadt, Germany), which is the most common calcium indicator after Fura-2. While Fura-2 was clearly not suitable to our needs since the keratin network has similar emission spectra, we opted for Indo-1, for which the excitation wavelength is about $340 \mathrm{~nm}$ and the emission wavelength is at $471 \mathrm{~nm}$ for no calcium or at $401 \mathrm{~nm}$ when calcium is present, respectively. Unfortunately, the spectrum of Indo-1 turned out to be also too broad so that we detected the signal from the keratin network in addition to the fluorescence intensity of the indicator. Our analysis was affected by the signal overlay, so that we were not able to obtain conclusive evidence for the calcium in our cells. As a consequence, we refer at this point to literature: Woo et al. have revealed that cells which were sheared by more than $0.009 \mathrm{~Pa}$ contain calcium concentrations that are higher than in cells to which no external forces are applied [107] (also supported by [108, 109]). In addition, the authors have found that both extracellular $\mathrm{Ca}^{2+}$ and intracellular $\mathrm{Ca}^{2+}$ contribute to the observed increase in calcium ion concentration. Interestingly, the authors have demonstrated that for mechanical shear of at least $0.016 \mathrm{~Pa}$ epithelial cells show an increase in ATP concentration, the energy-delivering molecule which also drives myosin motor activity. Flow-stimulated ATP release is furthermore dependent on $\mathrm{PCK} \zeta$ - the same kinase which influences the phosphorylation state of keratin under shear stress (see also section 2.2.4). An inhibition of $\mathrm{PCK} \zeta$ reduces the amount of 
flow-stimulated ATP release by about $75 \%$ 107.

Considering that we shear our cells with up to $0.27 \mathrm{~Pa}$, this indicates that there is probably a rise in calcium and ATP levels in our cells after shear-flow initiation, which hence supports our idea of increased myosin activity. The question that naturally arises is how this increased motor activity may be related to the behavior we observed for the keratin network under shear stress.

Since the actin network spans the cell in a cable-like structure (see figure 4.17 . [100, p. 783]), we propose that the increased myosin motor protein activity leads to an enhanced effective actin network rigidity, which is transmitted to the keratin bundles. The idea is also supported by a paper from Storm et al., which mentions that cytoskeletal components can regulate their stiffness through activation of motor protein contraction [41. The transmission between actin and keratin could, in turn, be achieved by direct coupling between the keratin and the actin network through coupling proteins in combination with simple confinement of the keratin that is imposed by the entire cytoskeleton surrounding the keratin bundles [73. Indeed, several proteins are known which couple actin and keratin, such as members of the plakin family $32,34,58$.

To experimentally confirm our hypothesis about the effect of cytoskeletal crosstalk on the observed keratin behavior, we inhibited the myosin II motor protein activity by treating the cells with the drug blebbistatin. Here, we found a distinct influence of myosin on the keratin dynamics since the bundle motion was highly reduced $4 \mathrm{~h}$ after drug addition (figure 4.15). Based on the detected influence of myosin on keratin dynamics, it seems plausible that if the myosin activity is altered by shear stress the observed KIF network dynamics should also change. Hence, we return to our initial proposition that the ultimately enhanced myosin activity due to shear stress likely increases the effective rigidity of the actin network and pre-stresses the cytoskeleton. This, in turn, should restrict the KIF bundle displacement and lead to the observed reduced motion of the KIF network.

Thus far, it is unclear whether phosphorylation of keratin takes place within the time period that we are interested in (5-20 min). Flitney et al. have found evidence that phosphorylation at least occurs within one hour after the application of shear stress [38]. They have also revealed that the state of phosphorylation of the keratin depends on the magnitude and the duration of stress: the larger and longer the stress, the more pronounced is the KIF phosphorylation. Since the shear forces that we apply to the cells are about five times smaller and they are established over shorter time periods, we assume that in our case the keratin network remains mostly in an unphosphorylated state. In accordance, Sivaramakrishnan et al. have 
(a)

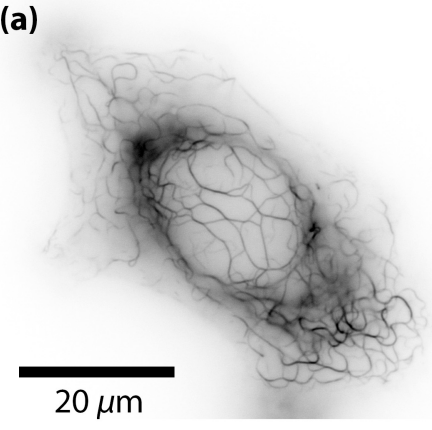

(d)

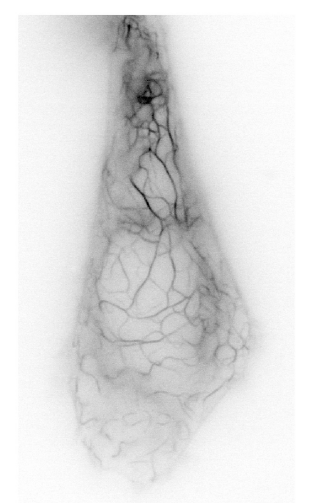

(b)

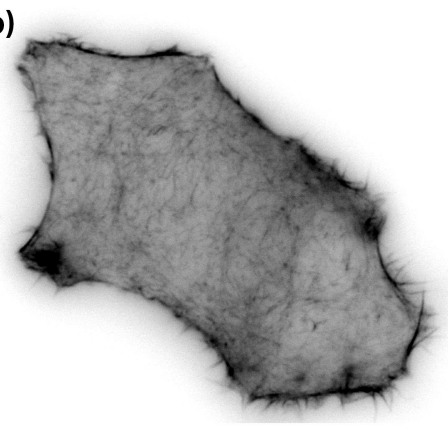

(e)

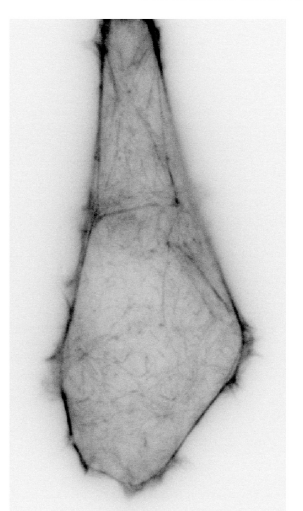

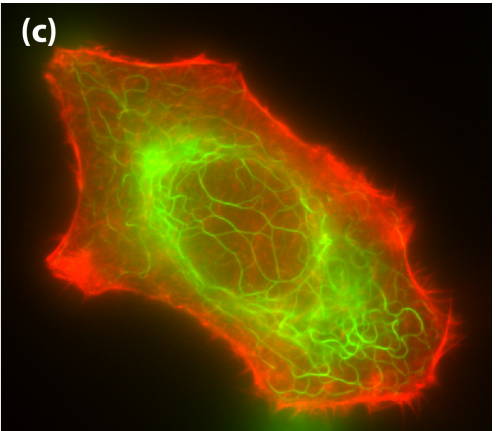

(f)

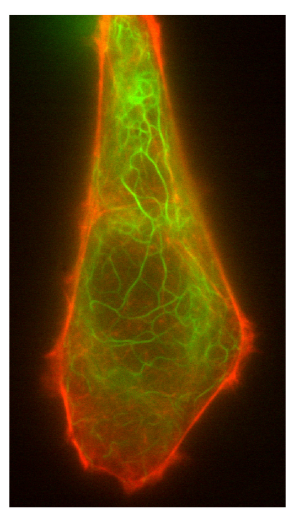

(g)

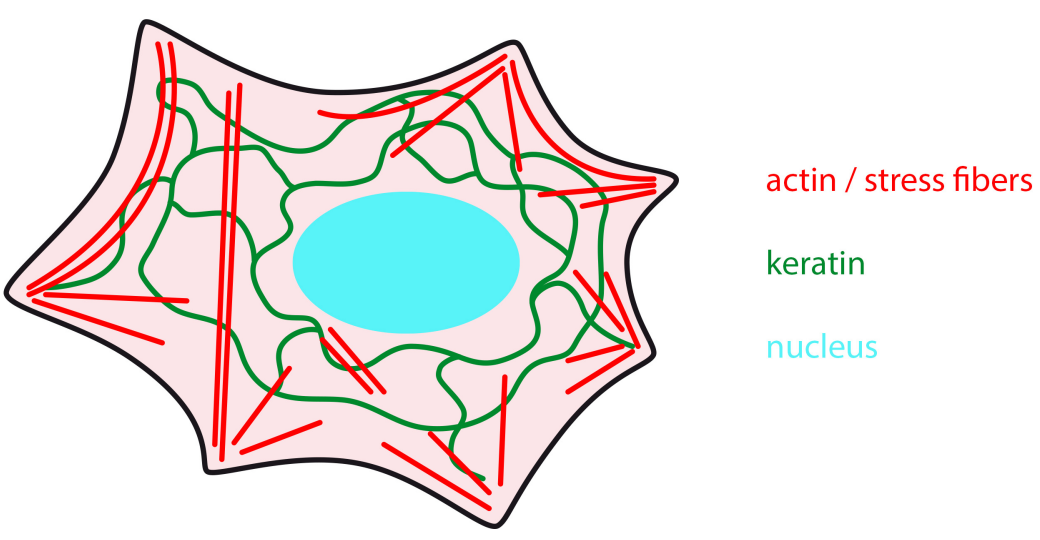

Figure 4.17: Epifluorescence images of two cells containing keratin $((\mathbf{a}),(\mathbf{d}))$ and actin networks ((b), (d)) and their composite images ((c), (f)). Images were taken after cells were left to settle on a glass coverslip for a day. The actin network spans the cell in a cablelike structure and is cross-linked with the keratin bundles. Actin network labeling in live cells was achieved by transfecting SK8/18-2 cells with LifeAct (ibidi GmbH, Martinsried, Germany) using electroporator 4D-Nucleofector (Lonza Cologne GmbH, Köln, Germany). (g) Sketch of a cell with a keratin network and actin stress fibers. The red background indicates that there is a remaining, finer actin network present in the cells apart from the stress fibers. 
observed in TEM images that the keratin network is unaltered upon application of shear stress to cells for short time periods of 5 to $30 \mathrm{~min}$ [72], which may indicate that the phosphorylation of the KIFs has either not started or the amount of time was not sufficient to have visible influence on the network structure. Although they have found out that tonofibrils are built within $1 \mathrm{~h}$ of shear exposition, they explain this finding by a merging of multiple KIF bundles. In addition, it has to be considered that the magnitude of the shear stress they have applied to their cells were much higher than for our case (1-3 $\mathrm{Pa}$ in comparison to $0.14 \mathrm{~Pa}$ wall shear stress).

While a reorganization of the KIF network is only clearly visible after the application of shear stress for about $1 \mathrm{~h}$ and we assume that a great part of the network in our short-timed experiments is still unphosphorylated, the phosphorylation process may nevertheless have already been initiated during our recordings. Since phosphorylated KIF bundles tend to integrate more keratin particles from the soluble pool, the bundle radius may consequently have increased for some bundles which conversely also influences the bending rigidity $\kappa$. In fact, the rigidity is expressed in terms of the elastic modulus of the material multiplied by the second moment of area which depends on the radius to the power of four in the case of a cylinder (see chapter 5). Thus, also small changes in the KIF bundle diameter may not be negligible when regarding the bundle rigidity. An increased bundle rigidity, in turn, would also contribute to our observed reduction in bundle motion.

In conclusion, we propose our observations to reflect a bridging response of the cell to external shear stress. As the cell has to ensure its integrity, it has to form an interim protection to outer mechanical influences until the process of network reorganization and reinforcement has been fully completed. Here, the effective tightening of the actin network, which is mediated by an increase in $\mathrm{Ca}^{2+}$ ions and is also transmitted to the keratin bundles, comes into play. This process can be established rather fast (within minutes) in comparison to the phosphorylation of the keratin network needed for reorganization (within hours) and thus provides a rapid method to withstand the external forces by reducing the extent of cellular deformation.

Our studies have shed light on the short-term response of cells to external forces, yet some new questions have also been raised. These relate especially to the interaction between the keratin and the actin network. In particular, it is of interest to compare the motion of the keratin bundles in the proximity of or distance to actin stress fibers. As the stress fibers at the cell center and the cell periphery are distinguishable from each other e.g. by the signaling cascades regulating their assembly (for details see 110,111]), it would also be informative to study the effect of stress fiber type on the keratin behavior. Furthermore, an investigation of the shear stress 
response of the cellular actin network itself, with regard to its location and dynamics, could lead to a deeper understanding of the mechanisms which are triggered by external forces. In fact, studies have revealed - primarily for endothelial cells that shear stress can induce the formation of actin stress fibers (e.g. 112, 113). In addition, the linkage between the actin and the keratin network in epithelial cells could be potentially changed by the presence of external forces. It could, for example, become stronger, which would increase the mechanical resilience of the cell. To clarify this point and ascertain whether cytoskeletal cross-talk is indeed involved in cellular shear stress response, a staining for e.g. plectin would be helpful. When comparing unsheared with sheared cells, it would be furthermore enlightening to study whether keratin, actin and a protein mediating the cross-talk co-localize in the case for cells which were exposed to external forces.

Overall, the investigation of the response of our cells to shear stress with different magnitudes could bring new insights. In this context, the question arises whether the phosphorylation of the KIF network is also present for very weak external forces or is solely triggered for stresses above a certain threshold. Both possibilities are conceivable, when taking into account that the enhanced resilience to mechanical influences that we have proposed may be sufficient for weak forces.

So far, we have studied shear stress response of cells on hard glass surfaces. It is, however, well known that cellular behavior changes if the rigidity of the substrate is altered (review in 114 ). For example, the formation of actin stress fibers is clearly observed for cells on glass surfaces, while hardly showing on soft substrates (e.g. [115]). In another study, Solon et al. have discovered, using atomic force microscopy (AFM), that cells adapt their elasticity to match the rigidity of the underlying substrate [116]. It would therefore be interesting to repeat our experiments on substrates with varying elasticities and analyze both how the keratin response to shear stress changes when actin stress fibers are absent and what role keratin plays in the adaptation of the cellular elasticity. 


\section{Buckling of Keratin Bundles}

In this chapter, we present and investigate buckling events which occur naturally in keratin bundles of live cells. The first section 5.1 explains the theory behind buckling in general, which is then extended for constrained buckling. Additionally, we introduce a coupling parameter to take the bundle entity comprised of KIFs into account. This parameter describes whether the filaments can slide against each other and quantifies this ability. Section 5.2 addresses the evaluation process of buckling events, where we present the way we determine the wavelength and the bundle diameter. Afterwards, the results are described: Section 5.3 contains information about the structural arrangement of filaments within keratin bundles and the results we have obtained from evaluating buckling events. The discussion of our data and a description of possible optimizations are finally presented in section 5.4 .

\subsection{Theoretical Background}

Beginning with the simplest model of Euler buckling, we introduce in the following the corresponding equations of motion and derive the total energy of the system, which consists of an elastic rod and an entity compressing the rod. We further use the energy as a starting point to deduce the equations of motion for the more complex case of constrained buckling. To this end, the total energy of Euler buckling is extended to include a contribution from an elastic matrix in which the rod is embedded. Before we arrive at the energy of constrained buckling (see figure 5.1 III), however, we need to review the basics of Euler buckling (as depicted in figure 5.1 II). 


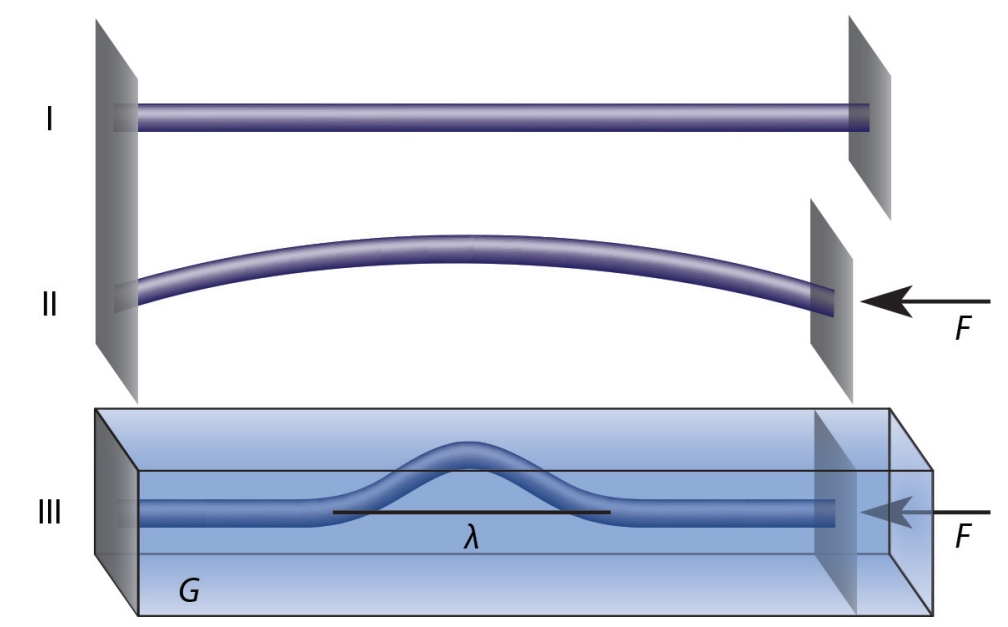

Figure 5.1: When an elastic rod is exposed to compression forces along its long axis it will buckle for sufficiently high forces (I $\rightarrow$ II). For a free rod, one long arc will appear (Euler buckling II). However, the shape of the deflection changes when the rod is embedded in a surrounding matrix (III). Here, the wavelength $\lambda$ depends not only on the bending rigidity of the rod but also on the shear modulus of the environment $G$.

\subsubsection{Euler Buckling and Bending Energy}

An extended description of the physics of buckling and bending of beams, bars and plates is given in reference [117]. Parts of this section are also derived from 85, 118, 119.

\section{The Bending Moment}

In analogy to Hooke's law the force which is required to stretch an elastic bar is expressed by

$$
F=E \cdot A \cdot \frac{\Delta L}{L},
$$

where $E$ describes a material property (elastic or Young modulus), $A$ represents the area of the cross-section of the bar and $\Delta L$ is the change of the original length $L$. In the analogy, the term $E A / L$ can be understood as the spring constant of the continuous medium of the elastic bar. The dependence on $A$ and $L$ becomes apparent if we consider the bar to consist of a network of identical, infinitely small springs which are longitudinally and laterally coupled. On the one hand, lateral coupling of springs increases the force needed to extend the springs in a way that is proportional to the number of springs per cross section and hence proportional to $A$. On the other hand, longitudinal coupling leads to an extension of the system that 
is the sum of extensions of all the longitudinally coupled springs. As a consequence, the system of longitudinally coupled springs is softer compared to the single springs by a factor that depends reciprocally on the number of springs along the length of the system, i.e. by a factor $1 / L$. From these considerations it follows that $E$ includes information about the 'density of infinitely small springs' along both the cross-section and the length of the elastic bar.

The force expression (5.1) is also the starting point for describing the bending of a bar. During a bending process layers of the material are deformed according to their position in the bar. The outer layers of the bent arc are stretched, while the inner layers closer to the point around which the arc bends are compressed. The two regions are divided by a neutral fiber which is characterized by retaining its length throughout the bending process (see figure 5.2.

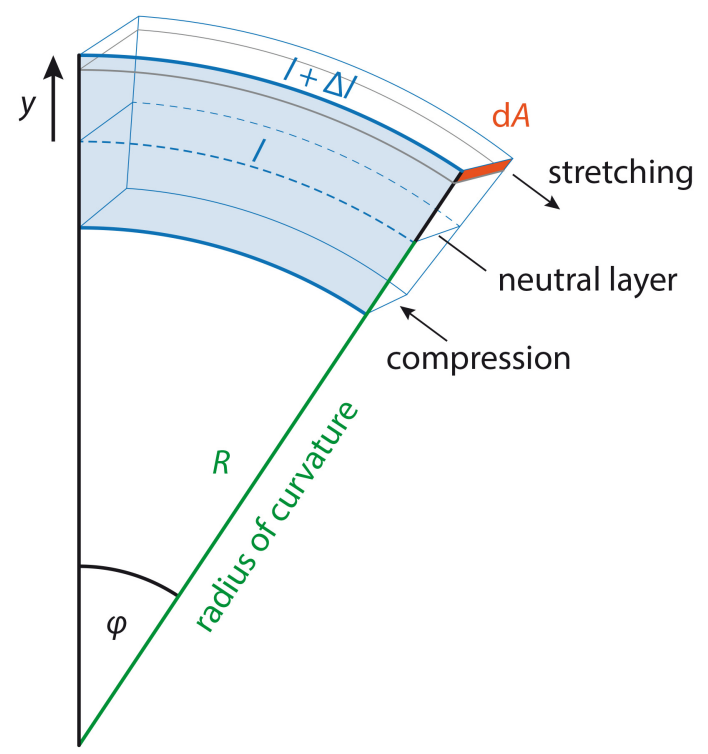

Figure 5.2: Bending process of a segment with length $l$ of a bar. The layer in the center of the segment follows a circular shape (radius $R$ ) and keeps its original length. Layers above and below the central one are stretched or compressed respectively.

In the following, a segment $l$ of the bent bar is regarded and a constituent thin layer with a distance $y$ to the neutral fiber and a cross-sectional area $\mathrm{d} A$ (figure 5.2 ). The force which is required to stretch this layer is expressed by

$$
\mathrm{d} F=E \cdot \mathrm{d} A \cdot \frac{\Delta l}{l}
$$

Since the arc of the bar segment can be expressed in terms of its radius of curvature $R$, that is $l=\varphi R$ or $l+\Delta l=\varphi(R+y)$, this can be rewritten as 


$$
\mathrm{d} F=E \cdot \mathrm{d} A \cdot \frac{y}{R} .
$$

The torque or bending moment for one layer is thus given by

$$
\mathrm{d} M=y \times \mathrm{d} F \stackrel{\text { here }}{=} y \cdot \mathrm{d} F=E \cdot \mathrm{d} A \cdot \frac{y^{2}}{R}
$$

and the total moment is obtained by integrating over all layers:

$$
M(x)=\int E \cdot \frac{y^{2}}{R} \cdot \mathrm{d} A=\frac{E}{R(x)} \int y^{2} \mathrm{~d} A=\frac{E \cdot I}{R(x)} .
$$

Here, the definition of the second moment of area $I$ is used - a parameter which describes the stability of a material against deformation as a function of the geometrical shape of the material. By introducing the bending rigidity $\kappa:=E \cdot I$ and the curvature $\rho:=\frac{1}{R}$ the bending moment is commonly written as

$$
M(x)=\kappa \rho(x)
$$

Equation (5.6) is generally valid in the linear elastic bending regime 1 . As detailed in the following, however, the equation can be further simplified if the deflections are small.

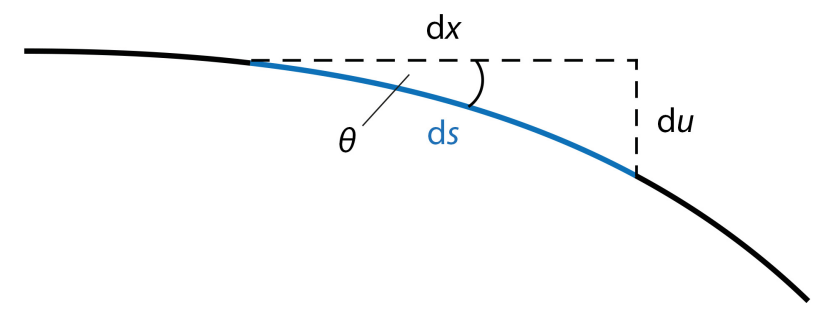

Figure 5.3: Sketch of a bent line to illustrate the parametrization notation.

With the notation as depicted in figure 5.3 , the following approximation can be made when the distance between two points $\mathrm{d} s$ is sufficiently small and thus the angle $\theta$ is small:

$$
\begin{aligned}
& \cos (\theta)=\frac{\mathrm{d} x}{\mathrm{~d} s} \approx 1 \Rightarrow \mathrm{d} s \approx \mathrm{d} x \\
& \sin (\theta)=\frac{\mathrm{d} u}{\mathrm{~d} s} \approx \theta \Rightarrow \theta \stackrel{5.77}{\approx} \frac{\mathrm{d} u}{\mathrm{~d} x}
\end{aligned}
$$

Taking this approximation into account, the curvature can be written as

$$
\rho=\frac{1}{R}=\frac{\mathrm{d} \theta}{\mathrm{d} s} \approx \frac{\mathrm{d}}{\mathrm{d} s}\left(\frac{\mathrm{d} u}{\mathrm{~d} s}\right)=\frac{\mathrm{d}^{2} u}{\mathrm{~d} s^{2}} \approx \frac{\mathrm{d}^{2} u}{\mathrm{~d} x^{2}} .
$$

\footnotetext{
${ }^{1}$ usually when the deflection process is reversible
} 
Consequently, the bending moment (equation (5.6) ) can be expressed by

$$
M(x)=E I \cdot \frac{\mathrm{d}^{2} u(x)}{\mathrm{d} x^{2}},
$$

where $u$ describes the transverse displacement of the rod as a function of the axial coordinate $x$.

\section{Equation of Motion and Critical Force}

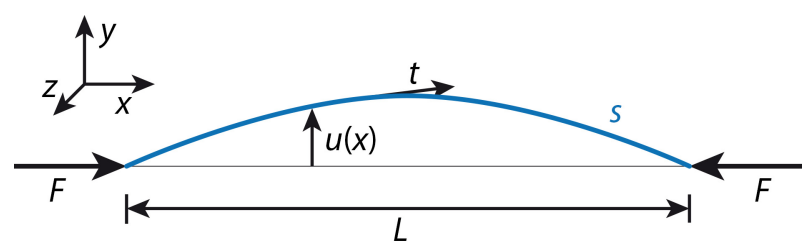

Figure 5.4: Euler buckling occurs when compression forces $F$ are applied to an elastic rod. As a result, the rod follows the shape of a long arc. Here, $u$ describes the deflection, $t$ the tangent vector along the arc with $\vec{t}=\frac{\partial \vec{r}}{\partial s}$ and $\vec{r}(s)$ is the position of a point on the arc described by an arc-length parameter $s$.

With this equation it is possible to find an expression for the shape of a deflected beam $u(x)$ which is buckled as a result of a compressive force $F$ (see also figure 5.4). To this end, we consider that the bending moment is generally given by $M=$ 'force' $\times$ 'lever'. Since the force in our case is perpendicular to the 'lever' $u$, we obtain with equation 5.10

$$
-F \cdot u(x)=E I \frac{\mathrm{d}^{2} u}{\mathrm{~d} x^{2}} \bigsqcup^{1}
$$

This differential equation is of the form

$$
\frac{\mathrm{d}^{2} u}{\mathrm{~d} x^{2}}+\varpi^{2} u(x)=0
$$

with $\varpi^{2}:=\frac{F}{E I}$ and can be solved using the ansatz $u=C_{1} \sin (\varpi x)+C_{2} \cos (\varpi x)$ with the boundary conditions that for $x=0$ and $x=L$ the deflection $u$ has to be zero, which is satisfied for $C_{1} \neq 0, C_{2}=0$ when a deflection is present. Since $\sin (\varpi L)=0$, the solution has to fulfill $\varpi L=n \pi$. Accordingly, the bending arc is represented by a sine, i.e. $u(x)=C_{1} \sin (n \pi x / L)$. The corresponding critical force $F_{\text {crit }}$ which is required for buckling is then given by

\footnotetext{
${ }^{1}$ The minus sign is a result of the fact that the bending moment, according to figure 5.4 , has to point in the negative $z$-direction for the arc to be bent in the direction of $u$.
} 


$$
F_{\text {crit }}=n^{2} \frac{\pi^{2} \cdot E I}{L^{2}}
$$

In practice, the first mode $(n=1)$ is the most important one since it represents the maximum load $F_{\text {crit }}^{n=1}$ a rod can take without showing buckling. However, also higher bending modes $(n>1)$ are possible and occur under certain boundary conditions (e.g. additional, perpendicular forces).

\section{Bending Energy for Euler Buckling}

In the following, an expression for the bending energy is derived. To this end, we first consider a segment of length $l$ of a bar and therein a small layer with crosssectional area $\mathrm{d} A$ (see figure 5.2). During bending, the bar is at first only bent a little, leading to an infinitesimally small curvature $\Delta \rho$ of the bar segment $l$. As a result, the layer with cross-sectional area $\mathrm{d} A$ within the bar segment undergoes a change in length by $\Delta l$, which yields an energy contribution

$$
\mathrm{d} F \cdot \Delta l=\underbrace{[E \cdot \mathrm{d} A \cdot y \cdot \Delta \rho]}_{\mathrm{d} F} \cdot \underbrace{[y \cdot \Delta \rho \cdot l]}_{\Delta l} .
$$

For the whole cross-section of the bar segment $l$ the energy contribution is obtained after integrating over the area:

$$
\int_{A} l \cdot \Delta \rho^{2} \cdot E \cdot y^{2} \mathrm{~d} A=l \Delta \rho^{2} \cdot E \cdot I
$$

So far, we have considered only the beginning of the bending of a bar, which is characterized by a small curvature $\Delta \rho$ in the segment $l$ of the bar. To obtain the total energy of the segment at the end of the bending process, we have to integrate over $\Delta \rho$ (from 0 to the final curvature $\rho$ at the end of the bending process). The parameter $\Delta \rho \rightarrow \mathrm{d} \rho$ is hereby present twice, since it is a variable of the force $\mathrm{d} F$ and the length $\Delta l$ :

$$
\begin{aligned}
\mathrm{d}^{2} H_{1, \text { bend }} & =l \mathrm{~d} \rho^{2} \cdot E \cdot I \\
H_{1, \text { bend }} & =\int\left(\int l \cdot E \cdot I \cdot \mathrm{d} \rho\right) \mathrm{d} \rho \\
& =\frac{1}{2} E \cdot I \cdot \rho^{2} \cdot l .
\end{aligned}
$$

Finally, we have to consider the whole rod with its total length $L$. To this end, we have to further integrate over the arc length $l$ of the bar segments $(l \rightarrow \mathrm{d} s)$ and obtain 


$$
\begin{array}{r}
H_{\text {bend }}=\int \frac{1}{2} \cdot E \cdot I \cdot \rho^{2} \mathrm{~d} s, \text { or } \\
H_{\text {bend }}=\frac{\kappa}{2} \int\left(\frac{\mathrm{d} \theta(s)}{\mathrm{d} s}\right)^{2} \mathrm{~d} s \approx \frac{\kappa}{2} \int\left(\frac{\partial^{2} u}{\partial x^{2}}\right)^{2} \mathrm{~d} x
\end{array}
$$

\subsubsection{Energy Contribution due to External Forces}

In the previous section we have derived the energy stored in a rod due to bending. However, to arrive at the total energy of our system, we also have to consider the energy associated with the forces acting on the rod. Without, at this point, specifying the nature of these forces, we can describe the corresponding energy contribution through the effect which is produced in the rod: The compressive force $f$ leads to buckling, which brings the ends of the rod closer together by a length $\Delta L$. The associated energy term can, in general, be expressed as

$$
H_{\mathrm{ext}}=f \cdot \Delta L
$$

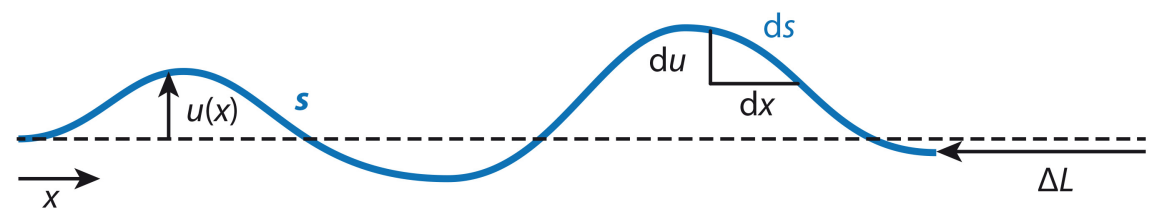

Figure 5.5: Sketch of a filament with arc length $s$ where the left end is fixed. Due to compressive forces, the filament shows a transverse deflection $u(x)$. As a result the end-to-end distance is reduced which is denoted by $\Delta L$.

The length $\Delta L$, in turn, is given by the difference between the contour length of the rod $s$ and its projected length on the axis of force generation ( $x$-axis in the following). According to figure 5.5, $s$ can be written as $\mathrm{d} s \approx \sqrt{\mathrm{d} x^{2}+\mathrm{d} u^{2}}$, i.e.

$$
s=\int \mathrm{d} x \sqrt{1+\left|\frac{\partial u}{\partial x}\right|^{2}} .
$$

Since the length of the projected filament on the $x$-axis is just given by the integral $\int \mathrm{d} x$ the reduction of the end-to-end distance is given by

$$
\Delta L=\int \mathrm{d} x\left(\sqrt{1+\left|\frac{\partial u}{\partial x}\right|^{2}}-1\right) .
$$

Using a Taylor expansion of the square root we obtain 


$$
\Delta L \approx \frac{1}{2} \int \mathrm{d} x\left|\frac{\partial u}{\partial x}\right|^{2} .
$$

Using the derived equation for $\Delta L$, we arrive at the final expression for the energy contribution of the force generator acting on the rod

$$
H_{\text {ext }}=\frac{1}{2} \int \mathrm{d} x f\left(\frac{\partial u}{\partial x}\right)^{2} .
$$

So far, we have not declared where the compressive force $f$, which ultimately causes the rod to bend, originates from. For cells, the forces that lead to buckling of cytoskeletal filaments and bundles are mostly generated by motor proteins. However, entropic forces can also play a role. In fact, the cytoskeletal components are constantly subject to thermal fluctuations [120]. Intuitively, this can be understood when thinking about a spaghetti which has been cooked. Left on its own, it usually acquires a wavy shape with a reduced end-to-end distance since there are more wavy configurations possible in comparison to the one configuration for a straight line. One has to invest energy to straighten the spaghetti and therefore reduce its entropy (see also discussion of entropic spring in section 4.5). This effect of a reduced end-to-end distance due to entropy considerations can be described as resulting from the action of entropic forces 1 . In our experiments, however, entropic forces play only a minor role since we focus on bundles which are very rigid. Indeed, we have observed that for dead cells the motion of bundles, as followed by epifluorescence microscopy, ceases completely. This, in turn, indicates that the buckling events seen in live cells are governed by force activators other than thermal ones, namely motor proteins (see also the estimation of required forces to deform a keratin network in section 4.5). For the following energy considerations it is, however, not necessary to know exactly were the compressive forces have their origins, as equation (5.25) is valid in general.

\subsubsection{Additional Energy Contribution for Embedded Rods}

For a complete description of a buckling process in a cell, we further have to consider the energy of the elastic deformation $H_{\text {el.def }}$ which is needed for the bending rod to deform the surrounding cytoplasm. Following references [73, 120], it is assumed that the deformation of the surrounding matrix is comparable with the behavior of an elastic spring. In analogy to Hooke's law the force (in our case per unit length of

\footnotetext{
${ }^{1}$ The forces needed to counterbalance the entropic forces and cause an extension in the filament have e.g. been described in reference [120].
} 
the rod) is given by a linear equation $(\sim \alpha u)$ and the energy is equal to a harmonic potential:

$$
H_{\text {el.def }}=\int \frac{1}{2} \alpha u^{2} \mathrm{~d} x
$$

with

$$
\alpha=\frac{4 \pi}{\ln (l / b)} G .
$$

where $l$ is a length that is on the order of the buckling wavelength $\lambda, G$ describes the shear modulus of the surrounding matrix and $b$ is the bundle diameter.

The parameter $\alpha$ is defined in analogy to the drag coefficient of a cylinder in hydrodynamics, where we have substituted the viscosity $\eta$ from hydrodynamics by the shear modulus $G$ for elasticity theory. As shown by Tiraldo et al. [118, 121], the drag coefficient $\varsigma$ of a cylinder, which is moving through a fluid with its long axis oriented perpendicularly to the direction of motion, is given by

$$
\varsigma=\frac{4 \pi \eta L}{\ln (L / 2 r)+0.84} .
$$

Here, $L$ describes the length of the cylinder and $r$ the radius. Since we are calculating forces $\alpha u$ or energies $\frac{1}{2} \alpha u^{2}$ per length segment $\mathrm{d} x$ for every deflection $u$, $\alpha$ in equation (5.27) is not a function of length, in contrast to $\varsigma$. The denominator in $\varsigma$ is furthermore approximated in $\alpha$ by the logarithm of the ratio between buckling wavelength (in place of the cylinder length) and the diameter of the bundle.

In the following, we show the analogy between elasticity theory and hydrodynamics which allows us to adapt equation (5.28) to $\alpha$ as detailed above. For this purpose, we first review some of the principles from elasticity theory based on the descriptions given in reference [119, chapter 1.4].

The basic equation in elasticity theory which describes all possible tensions $\sigma_{i j}$ acting on an infinitesimally small cubic volume $\mathrm{d} V$ at a position $\vec{x}$ within an elastic medium is given by

$$
\sigma_{i j}=3 K \underbrace{\left(\frac{1}{3} \delta_{i j} \sum_{k} \frac{\partial u_{k}}{\partial x_{k}}\right)}_{\text {homogenous dilatation }}+2 G \underbrace{\left(\frac{1}{2}\left(\frac{\partial u_{i}}{\partial x_{j}}+\frac{\partial u_{j}}{\partial x_{i}}\right)-\frac{1}{3} \delta_{i j} \sum_{k} \frac{\partial u_{k}}{\partial x_{k}}\right)}_{\text {pure shear }} .
$$

Here, the index $i$ of $\sigma_{i j}(\vec{x})$ describes the direction in which a tension force points and $j$ refers to the normal vector of the area on the infinitesimal cube on which the force acts. Furthermore, $\vec{u}$ is the deformation vector for the cubic volume $\mathrm{d} V$ at 
position $\vec{x}=\left(x_{1}, x_{2}, x_{3}\right)$. The parameters $K$ (bulk modulus) and $G$ (shear modulus) are material constants. They can be expressed in terms of the elastic modulus $E$ as shown in the following, where $\nu$ describes Poisson's ratio $\left(\nu=-\frac{\Delta w / w}{\Delta l / l}\right.$, with the relative change in width $w$ and length $l$ ):

$$
K=\frac{E}{3(1-2 \nu)}, \quad G=\frac{E}{2(1+\nu)} .
$$

The first term in equation 5.29 describes pure dilation/compression while the second one represents pure shear, meaning shear in the absence of volume changes. As evidenced by equation (5.29) the second term itself is composed of two parts. The first describes every shear deformation $\left(\frac{\partial u_{i}}{\partial x_{j}}+\frac{\partial u_{j}}{\partial x_{i}}\right)$ independently of whether it is accompanied by volumetric changes or not. The second part is then included to subtract possible volumetric changes to arrive at the expression for pure shear. As we are interested in shear deformations in general, the expression 5.29 is reformulated while introducing the Lamé constant $\lambda_{l}=K-\frac{2}{3} G$

$$
\sigma_{i j}=G\left(\frac{\partial u_{i}}{\partial x_{j}}+\frac{\partial u_{j}}{\partial x_{i}}\right)+\lambda_{l} \delta_{i j} \sum_{k} \frac{\partial u_{k}}{\partial x_{k}}
$$

In equilibrium, all forces acting within the elastic medium due to tensions $\sigma_{i j}$ have to be zero. This requirement is fulfilled when

$$
\vec{\nabla} \sigma_{i j}=0
$$

Switching from elasticity theory to hydrodynamics, we return to section 4.1.1, where we have introduced the Navier-Stokes equation as the equation describing the motion of a fluid flow, i.e.

$$
\rho \frac{\mathrm{d} \vec{u}}{\mathrm{~d} t}=\vec{\nabla} \underline{\underline{\sigma}}+\vec{f}_{\text {ext }} .
$$

Note, that the parameter $\vec{u}$ now describes a velocity instead of an elastic deformation. Furthermore, the tension forces (tensor $\underline{\underline{\sigma}}$ ) are usually written as a combination of pressure and viscous forces:

$$
\vec{\nabla} \underline{\underline{\sigma}}=-\nabla p+\eta \nabla^{2} \vec{u}
$$

However, the equation above is valid only for incompressible fluids. The full expression of $\underline{\underline{\sigma}}$, accounting also for compressible fluids, is given by 


$$
\underline{\underline{\sigma}}=-\delta_{i j} p+\eta\left(\frac{\partial u_{i}}{\partial x_{j}}+\frac{\partial u_{j}}{\partial x_{i}}\right)+\underbrace{\lambda \delta_{i j} \sum_{k} \frac{\partial u_{k}}{\partial x_{k}}}_{\text {here }=0} .
$$

where the last term describes the divergence of $\vec{u}$, which is zero for incompressible fluids due to conservation of mass.

In the absence of external forces $\left(\vec{f}_{\text {ext }}=0\right)$ and for low Reynolds numbers $\left(\rho \frac{\mathrm{d} \vec{u}}{\mathrm{~d} t}\right.$ neglectable), we arrive at the Stokes equation $\vec{\nabla} \underline{\underline{\sigma}}=0$, i.e.

$$
0=-\nabla p+\eta \nabla^{2} \vec{u}=\vec{\nabla} \underline{\underline{\sigma}}
$$

As drag coefficients are usually calculated based on equation 5.36 for the limit of low Reynolds numbers, we have thus established the analogy between the theory of elasticity (equation (5.32) and hydrodynamics (equation (5.36). By comparing equation (5.31) with (5.35) we, moreover, identify $G$ from elastic theory to be the analog of $\eta$ from hydrodynamics. Note, that in fluid dynamics, $\underline{\underline{\sigma}}$ contains an additional term describing the fluid pressure. For fluids, the surface forces which are acting on a small volume element are not only described by inner tensions but also by the pressure exerted by the surrounding fluid. Assuming the pressure contribution to the hydrodynamic drag coefficient to be negligible for small Reynolds numbers, we thus obtain the expression of $\alpha$ according to reference 120.1 .

\subsubsection{Constrained Buckling}

Finally, we obtain the following equation for the total energy

$$
\begin{aligned}
H & =H_{\text {bend }}-H_{\text {ext }}+H_{\text {el.def }} \\
H & =\frac{1}{2} \int \mathrm{d} x\left[\kappa\left(\frac{\partial^{2} u}{\partial x^{2}}\right)^{2}-f\left(\frac{\partial u}{\partial x}\right)^{2}+\alpha u^{2}\right]
\end{aligned}
$$

which contains the bending energy (section 5.1.1), the external force energy (section 5.1.2) and the energy which is needed to deform the surrounding matrix (section 5.1.3). Here, the term corresponding to the external force energy has a minus sign since this energy is applied to the system (e.g. motor proteins exert force on the KIF bundle) and the other energy terms describes the response of the system (i.e. the bundle gets curved and pushes against the surrounding matrix).

\footnotetext{
${ }^{1}$ The expression of $\alpha$ was first introduced in reference 73 ; however, $G$ is mentioned in this paper as being an elastic modulus.
} 


\section{Wavelength of Constrained Buckling}

Assuming that the system is in equilibrium, we can deduce the buckling wavelength from the energy equation $(5.38)$ by a variational approach as described in reference [73]. In equilibrium, the energy is in a local minimum and does not change for small changes in the transverse displacement of the rod, i.e.

$$
\frac{\delta H}{\delta u}=0
$$

For a given functional $H(u(x))=\int F\left(u(x), u^{\prime}(x), u^{\prime \prime}(x), x\right) \mathrm{d} x$ of higher order derivatives with $u^{\prime}:=\frac{\mathrm{d} u}{\mathrm{~d} x}$, variation theory states according to reference $[122, \mathrm{p} .577]$ that equation $(5.39)$ is satisfied for:

$$
-\frac{\mathrm{d}^{2}}{\mathrm{~d} x^{2}} \frac{\partial F\left(u, u^{\prime}, u^{\prime \prime}, x\right)}{\partial u^{\prime \prime}}+\frac{\mathrm{d}}{\mathrm{d} x} \frac{\partial F\left(u, u^{\prime}, u^{\prime \prime}, x\right)}{\partial u^{\prime}}-\frac{\partial F\left(u, u^{\prime}, u^{\prime \prime}, x\right)}{\partial u}=0
$$

Applied to the energy equation (5.38), we obtain

$$
\begin{aligned}
-\frac{\mathrm{d}^{2}}{\mathrm{~d} x^{2}}\left(\kappa u^{\prime \prime}\right)+\frac{\mathrm{d}}{\mathrm{d} x}\left(-f u^{\prime}\right)-\alpha u & =0 \\
\kappa u^{\prime \prime \prime \prime}+f u^{\prime \prime}+\alpha u & =0
\end{aligned}
$$

The solution of this equation is of the form $u \sim e^{i k x}$ where the wave vector $k$ is introduced. Thus, we obtain

$$
\kappa k^{4}-f k^{2}+\alpha=0
$$

which is solved by

$$
k^{2}=\frac{1}{2 \kappa}\left(f \pm \sqrt{f^{2}-4 \kappa \alpha}\right) .
$$

The solutions for $k$ are only real for forces which are above a threshold of $f_{\text {crit }}=$ $2 \sqrt{\kappa \alpha}$ which is in analogy to the threshold for Euler buckling (see equation (5.13)). The corresponding buckling wavelength $\lambda=2 \pi / k$ is given by

$$
\lambda=2 \pi\left(\frac{\kappa}{\alpha}\right)^{1 / 4}
$$




\subsubsection{Bending of Coupled-Rods}

The theory of constrained buckling has to be adapted further to be applicable to the deflection of multiple subrods which are combined into a bundle. When investigating buckling events of bundles, the interactions of the subrods with each other have to be taken into account. As also depicted in figure 5.6, two extreme cases are possible: First, if the cross-linkages between the subrods are fixed and rigid, the strong coupling results in a bundle which acts itself as one single rod with a greater diameter. For such a strongly intralinked bundle, a deflection leads to a compression of the subrods below the central layer, as well as a stretching of the subrods above the central layer. The central layer alone remains neutral, meaning that its length remains unchanged during the deflection process (figure 5.6a). Second, if the linkage between the bundle constituents is flexible, it allows for sliding of the subrods against each other. Here, a deflection does not lead to an alteration in length of any subrod. Every constituent filament of the bundle has the same length as in the undeflected state (figure $5.6 \mathrm{~b}$ ).
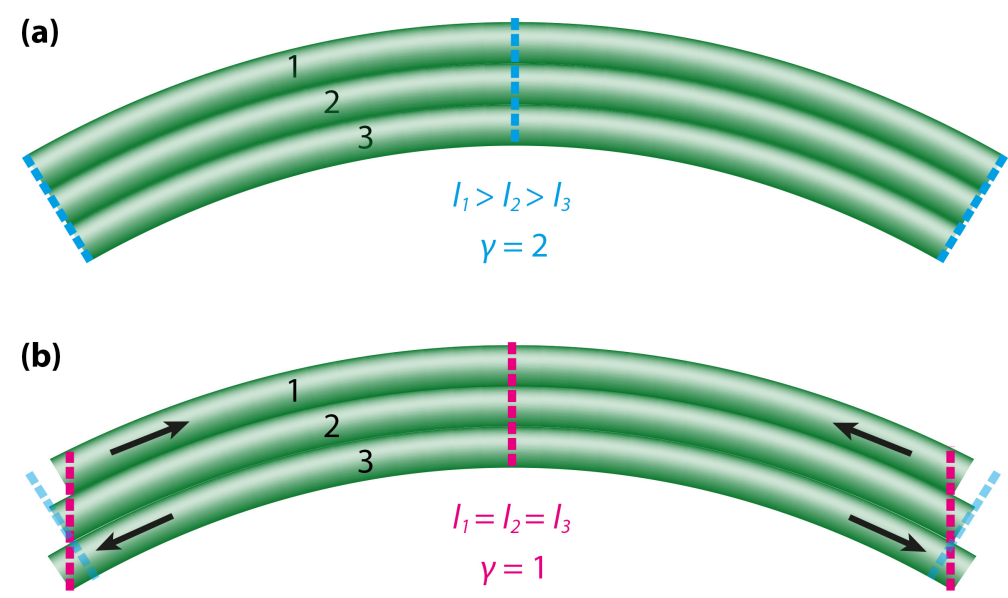

Figure 5.6: The buckling behavior of a bundle is dependent on the coupling between bundle components (subrods). (a) For strong linkage, the bundle acts as a single rod, where the top subrod 1 is stretched and the bottom subrod 3 is compressed. (b) When the constituents of the bundle are allowed to shift against each other, every subrod retains its original length.

When the subrods within a bundle are strongly coupled, additional energy is required for the alteration of the subrod lengths. As the energy of the bent bundle is dependent on the rigidity $\kappa$ of the bundle (equation $(5.19)$ ), the latter parameter is consequently expected to be also dependent on the strength of cross-linkage between the bundle constituents. To specify this dependency a coupling parameter $\gamma$, which describes the strength of linkage within a bundle [123], is introduced in the following: 
As described in a previous section, the bending rigidity of a rod in general is given by its elastic modulus $E$ and its second moment of area $I$

$$
\kappa=E \cdot I .
$$

As the persistence length $L_{P}$ of a rod expresses the bending rigidity in terms of the thermal energy, we further obtain

$$
L_{\mathrm{P}}=\frac{\kappa}{k_{\mathrm{B}} T}
$$

Accordingly, the persistence length of an individual, cylindrical filament with radius $R_{\mathrm{F}}$ is given by $L_{\mathrm{PF}}=I_{F} \cdot \frac{E}{k_{\mathrm{B}} T}$, where the second moment of area is represented by $I_{\mathrm{F}}=\frac{\pi}{4} R_{\mathrm{F}}^{4}$. In the limit of no coupling, the persistence length of the bundle $L_{\mathrm{PB}}$ is obtained by the sum of the persistence lengths of all constituting filaments:

$$
L_{\mathrm{PB}}=\sum_{i=1}^{N} L_{\mathrm{PF}, i}=N \cdot L_{\mathrm{PF}} .
$$

In the opposite limit of a completely coupled bundle, all subrods within a bundle act together as a unit, so that the second moment of area turns into that of a single cylinder with a radius corresponding to the bundle radius $R_{\mathrm{B}}$, i.e. $I_{\mathrm{B}}=\frac{\pi}{4} R_{\mathrm{B}}^{4}$. This leads to a persistence length of

$$
L_{\mathrm{PB}}=\frac{\kappa}{k_{\mathrm{B}} T}=\frac{E \cdot I_{\mathrm{B}}}{k_{\mathrm{B}} T}=\frac{E \cdot \frac{\pi}{4} R_{\mathrm{B}}^{4}}{k_{\mathrm{B}} T} .
$$

Since the cross-sectional area of the bundle $A_{\mathrm{F}}$ is given by the sum of the filament cross-sections $A_{\mathrm{F}}$, i.e. $A_{\mathrm{B}}=\pi R_{\mathrm{B}}^{2}=N \cdot A_{\mathrm{F}}=N \cdot \pi R_{\mathrm{F}}^{2}$, this implies $N \cdot R_{\mathrm{F}}^{2}=R_{\mathrm{B}}^{2}$. Thus, we obtain for the second moment of area for a strongly coupled bundle $I_{\mathrm{B}}=$ $\frac{\pi}{4} R_{\mathrm{B}}^{4}=N^{2} \cdot \frac{\pi}{4} R_{\mathrm{F}}^{4}$, which leads to a persistence length of the bundle of

$$
L_{\mathrm{PB}}=N^{2} \cdot L_{\mathrm{PF}} .
$$

The strength of coupling is expressed in equation (5.49) in terms of the exponent to the number of filaments $N$ in a bundle. As demonstrated by the discussion above for the two limiting cases for coupling, this exponent has to range between one and two. In summary, the persistence length of a bundle for any degree of coupling between the individual filaments is given by

$$
L_{\mathrm{PB}}=N^{\gamma} \cdot L_{\mathrm{PF}}, \quad \gamma \in[1,2] .
$$




\subsubsection{Constrained Buckling of Bundles}

As described in section 5.1.4 the buckling events which are observable in cells are determined by the properties of the rod (i.e. its bending rigidity $\kappa$ ) and the stiffness of the surrounding medium (expressed by the parameter $\alpha$ ). In the following, the relation of the buckling wavelength is extended based on the insights gained for the coupling between several subrods within a bundle in section 5.1 .5 .

According to equation (5.44), the wavelength of a buckling event of a single rod is given by

$$
\lambda=2 \pi\left(\frac{\kappa}{\alpha}\right)^{1 / 4}
$$

where $\kappa$ represents the bending rigidity of the buckled rod. Using expression 5.46 we reformulate $\kappa$ for a buckled bundle which leads to

$$
\lambda=2 \pi\left(\frac{L_{\mathrm{PB}} \cdot k_{\mathrm{B}} T}{\alpha}\right)^{1 / 4},
$$

where $L_{\mathrm{PB}}$ describes the persistence length of the bundle. Finally, we use relation (5.50) and introduce the coupling parameter $\gamma$. This leads to the following expression of the buckling wavelength of a bundle:

$$
\lambda=2 \pi\left(\frac{N^{\gamma} \cdot L_{\mathrm{PF}} \cdot k_{\mathrm{B}} T}{\alpha}\right)^{1 / 4} .
$$

\subsection{Evaluation of Buckling Events}

The evaluation of the KIF bundle deflection is based on several steps which are explained in the following. The image acquisition itself is performed using confocal microscopy. As confocal microscopy has an enhanced image resolution in comparison to common epifluorescence, our choice of technique ensures good imaging of the keratin bundle shape (see section 3.6). After the image acquisition, the image stack is manually searched for buckling events. Here, it is necessary to distinguish between deflections which are caused by compressive forces and those which are a result of a transverse pulling force at one point on the keratin bundle. As indications for the latter we have considered deflection shapes that extend from network node to network node. These shapes are characterized by a high curvature at one point and show no clear turning points for the curvature toward the nodes, i.e. between the point of highest curvature and the nodes the bundles follow approximately straight 
(a)

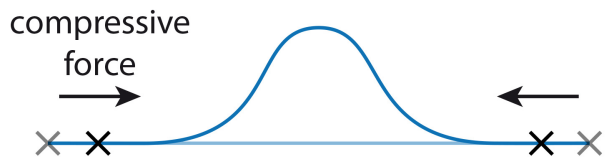

(b)

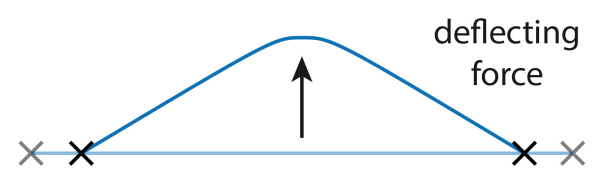

Figure 5.7: For the identification of buckling events which are caused by compressive forces, we investigate the shape of the bundle. (a) When nodes are shifted towards each other and the contour shows distinct turning points we assume a buckling event. (b) By contrast, we abstain from the evaluation of curves without clear changes in the curvature as they may be a result of a direct deflection due to pulling forces.

lines (see figure 5.7). Deflections of this form were sorted out prior to data evaluation. Although this criterion should satisfactorily filter out the deflections which are caused by compressive forces and thus represent true buckling events, there is, nevertheless, no ultimate guarantee that we solely analyze buckling waveforms.

\subsubsection{Determination of the Buckling Wavelength}

The evaluation of the buckling wavelength is performed with a self written Matlab script. An example of the evaluation process is depicted in figure 5.8 . Here, the deflection occurs near a branching point in the KIF network which poses an additional challenge and shows best the significance of the steps taken during data processing.

After a buckling event has been identified (figure $5.8 \mathrm{a}-\mathrm{c}$ ), the evaluation process starts with an interactive selection of the region of interest (ROI) in the microscopy image (figure $5.8 \mathrm{~d}$ ). The image is then reduced to that ROI which just contains the bundle that show the buckling event. The ROI is displayed in a jet colormap in all subsequent evaluation steps to enable a better visualization of the intensity depth.

Next, the ROI is filtered using a 2D Gaussian. This convolution enhances the homogeneousness of the imaged structures and reduces the amount of exceptionally bright or dark pixels caused by noise during image acquisition (see figure 5.8.). Afterwards, the ROI is converted into a binary image where the keratin structure is the only remaining feature of the image (figure $5.8 \mathrm{f}$ ). To this end, a function in the Matlab library, which uses a method of Otsu [124, is utilized for dynamic threshold calculation. The obtained image is then treated with a thinning algorithm [125], which is also included in the Matlab library. As a result, the structure in the binary image is reduced to a minimally connected stroke representing a one pixel line of the original shape (figure $5.8 \mathrm{~g}$ ).

In a subsequent step, the obtained one-pixel-line is displayed as an overlay on 


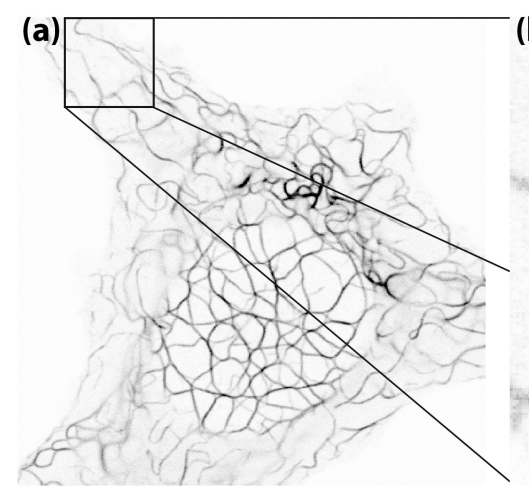

(b)

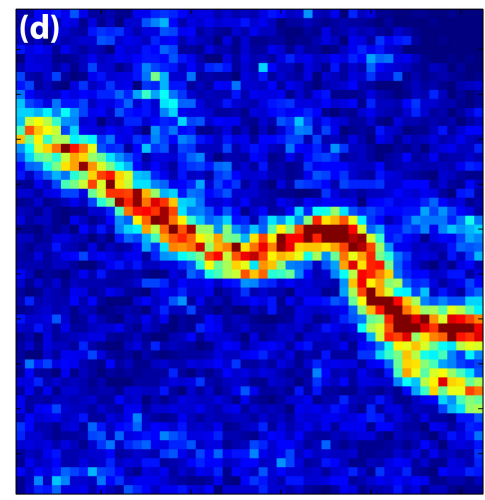

(g)

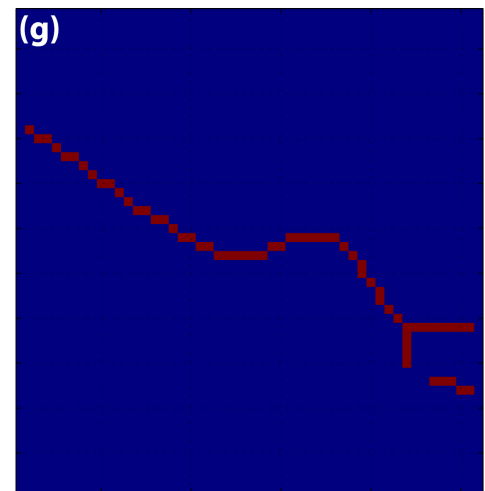

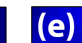

(c)

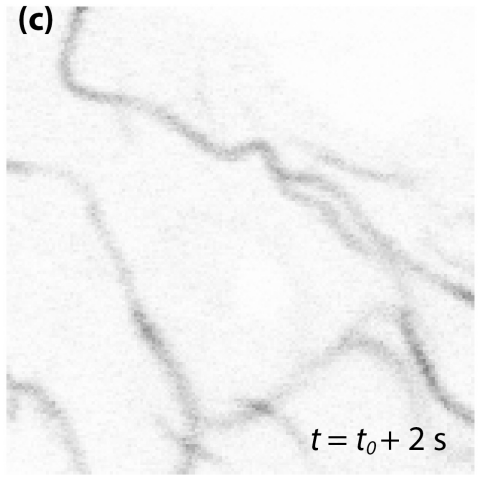

(f)
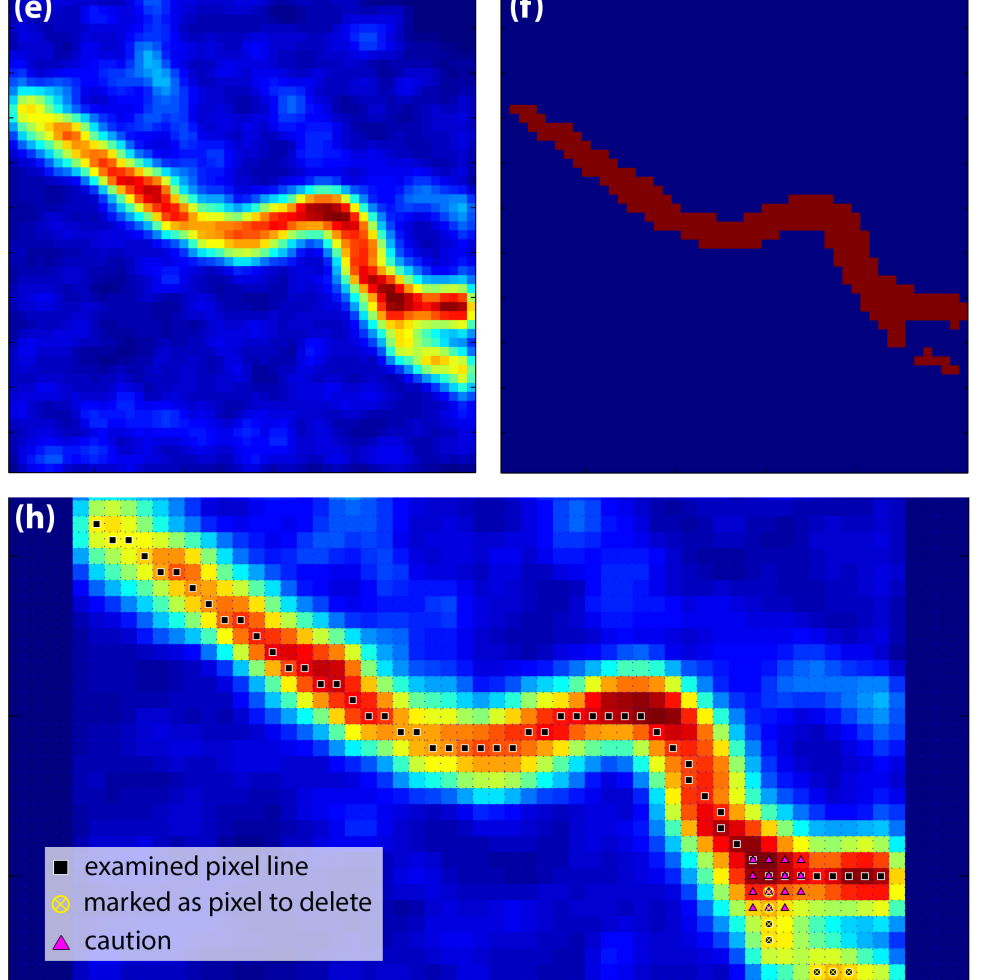

Figure 5.8: After identifying a buckling event in the confocal microscopy image stack $((\mathbf{a}),(\mathbf{b}) \rightarrow(\mathbf{c}))$, a self written Matlab script is used to evaluate the buckling wavelength. (d) To this end, a region of interest is cut out of the raw image which is subsequently displayed using a jet colormap to enhance visibility. (e) Afterwards, the image is convoluted with a Gaussian. This step aims for a reduction in image noise which is important for a more homogeneous result of the following binarizing process (f). The structure of the KIF bundle in the binarized image is then reduced to a one-pixel-line (g). Next, unnecessary pixels are marked by the user in an interactive step. This includes image noise which was not excluded in the binarizing process (not present in this example) and additional lines extending from branching points in the bundle network (h). For subsequent fitting the existence of a continuous, unique one-pixel-line with a distinct starting point and end point is required. 
top of the original image. Here, the user can interactively mark pixels which are unintended for further curve fitting. Examples of which include pixels representing additional keratin bundles apart from the one of interest, or noise with uncommonly high intensities which was thus not excluded by the previous thresholding. In the example depicted in figure 5.8, the second keratin bundle protrudes, with lower intensities, from the branching point in the KIF network. Consequently, it is marked and thus excluded from further analysis. To aid the user in this step, the script automatically reveals branching points in the network and calls for user intervention.

For further data processing, only a single dedicated one-pixel-line is allowed to remain. This requirement is essential since the following fitting of the keratin bundle shape is only possible along one pathway. As preparation for the fitting procedure, the pixel line is indexed. To this end, a script was written which first searches for an end point of the one-pixel-line (a $3 \times 3$ matrix in which the sum of the entries is 2 and one pixel is in the center). Having identified an end point the script then follows the line by searching for a pixel with a value of 1 in a $3 \times 3$ matrix around the current position. The new pixel has to satisfy the additional restriction that it is not the current one, nor the previous one considered.

The actual fitting is not directly performed on the obtained $(x, y)$ coordinates after indexing since the path represented by the one-pixel-line cannot always be described by an analytical function of the form $y=\mathrm{f}(x)$ (an example, where such an analytical representation fails, would be a vertical line). Instead, we parametrize the curve $[x(s), y(s)]$ with the arc length $s$. For this purpose, the relative changes between the absolute coordinates of the pixels on the pixel-line $d_{i}=\sqrt{\left(x_{i+1}-x_{i}\right)^{2}+\left(y_{i+1}-y_{i}\right)^{2}}(i=$ pixel index $)$ are calculated, where $d_{i}$ is equal to 1 if two neighboring pixels are next to each other or equal to $\sqrt{2}$ in case they are located diagonally from each other. The arc length $s$ is then gradually incremented along the one-pixel-line according to $s_{i+1}=s_{i}+d_{i}$. For a given pixel index $i$ the representation $s_{i}=s(i)$ is unique (see figure $5.9 \mathrm{a}$ ) and more importantly so is the representation $f\left(s_{i}\right)=\left[x\left(s_{i}\right), y\left(s_{i}\right)\right]$, where $\left[x\left(s_{i}\right), y\left(s_{i}\right)\right]$ constitutes the coordinates of the pixel $p_{i}$.

Following parametrization, we fit the pixel line $[x(s), y(s)]$ with a smoothing spline fit of Matlab which consists of connected polynomial functions, each of which is defined on a small interval within the total range of $s$. Using the Matlab spline tool it is furthermore possible to set a certain tolerance. Accordingly, the final fit is not allowed to deviate from the input data by more than the maximum distance of the preset tolerance. The obtained spline fit is then analytically differentiated twice to gain access to the curvature. Afterwards, the parameter $s$ is resampled to 
(a)

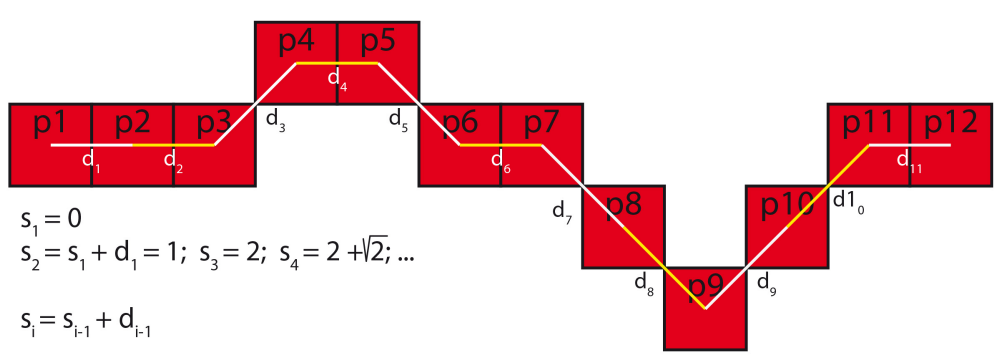

(b)

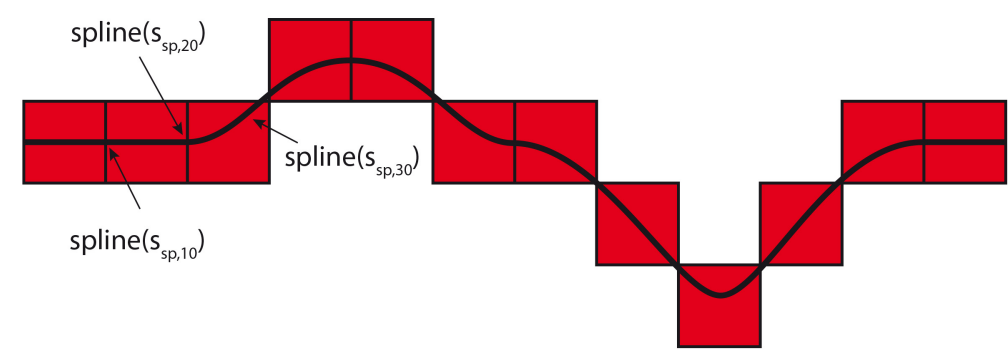

Figure 5.9: (a) Example for a one-pixel-line as represented by red boxes. According to the geometry of the CCD camera employed for image acquisition, pixels can be located either aside one another or diagonally from each other. Consequently, the distance $d$ between two neighboring pixels on the pixel line can only be 1 or $\sqrt{2}$. The sum of the distances $d$ from the start point of the pixel line to a given position $i$ is calculated to obtain the arc length $s_{i}=s(i)$. This arc length $s$, in turn, is used to parametrize the one-pixel-line. (b) Spline fit is evaluated as a function of the parameter $s_{\mathrm{sp}}$. Here, again the arc length $s_{\mathrm{sp}}$ of the spline fit is used to parametrize the spline curve. Additionally, $s_{\mathrm{sp}}$ follows the spline fit equidistantly with sub-pixel steps of $1 / 10$ pixel.

evaluate the spline fit and the curvature function in steps of sub-pixel accuracy. To this end, a new parameter $s_{\mathrm{sp}}$ is introduced which is defined as a vector ranging from 0 to the last value of $s\left(\right.$ i.e. $\left.s_{N}\right)$ in small steps of $1 / 10$ of a pixel. The steps in $s_{\mathrm{sp}}$ are thus - contrary to the steps in $s$ - equidistant. The evaluation of the spline curve as a function of $s_{\mathrm{sp}}$ leads to an array $\operatorname{spline}\left(s_{\mathrm{sp}}\right)=\left[x_{\mathrm{sp}}\left(s_{\mathrm{sp}}\right), y_{\mathrm{sp}}\left(s_{\mathrm{sp}}\right)\right]$ (see figure $5.9 \mathrm{~b}$, which contains the coordinates to densely packed points on the spline fit. By calculating the corresponding second derivative we obtain the curvature vectors $\left[\operatorname{curv}_{x}\left(s_{\mathrm{sp}}\right), \operatorname{curv}_{y}\left(s_{\mathrm{sp}}\right)\right]$ at the points $\left[x_{\mathrm{sp}}\left(s_{\mathrm{sp}}\right), y_{\mathrm{sp}}\left(s_{\mathrm{sp}}\right)\right]$. Based on these data, the absolute values for the curvatures are calculated $\left(\operatorname{curv}_{\mathrm{abs}}=\sqrt{\operatorname{curv}_{x}^{2}+\operatorname{curv}_{y}^{2}}\right)$ and plotted in a graph against the coordinates $\left[x_{\mathrm{sp}}\left(s_{\mathrm{sp}}\right), y_{\mathrm{sp}}\left(s_{\mathrm{sp}}\right)\right]$ (see figure 5.10). For easier visualization, the plot furthermore contains projections of the absolute curvature onto the $x$ - and $y$-axis.

Since local maxima of curvature also represent the start and end points for the 


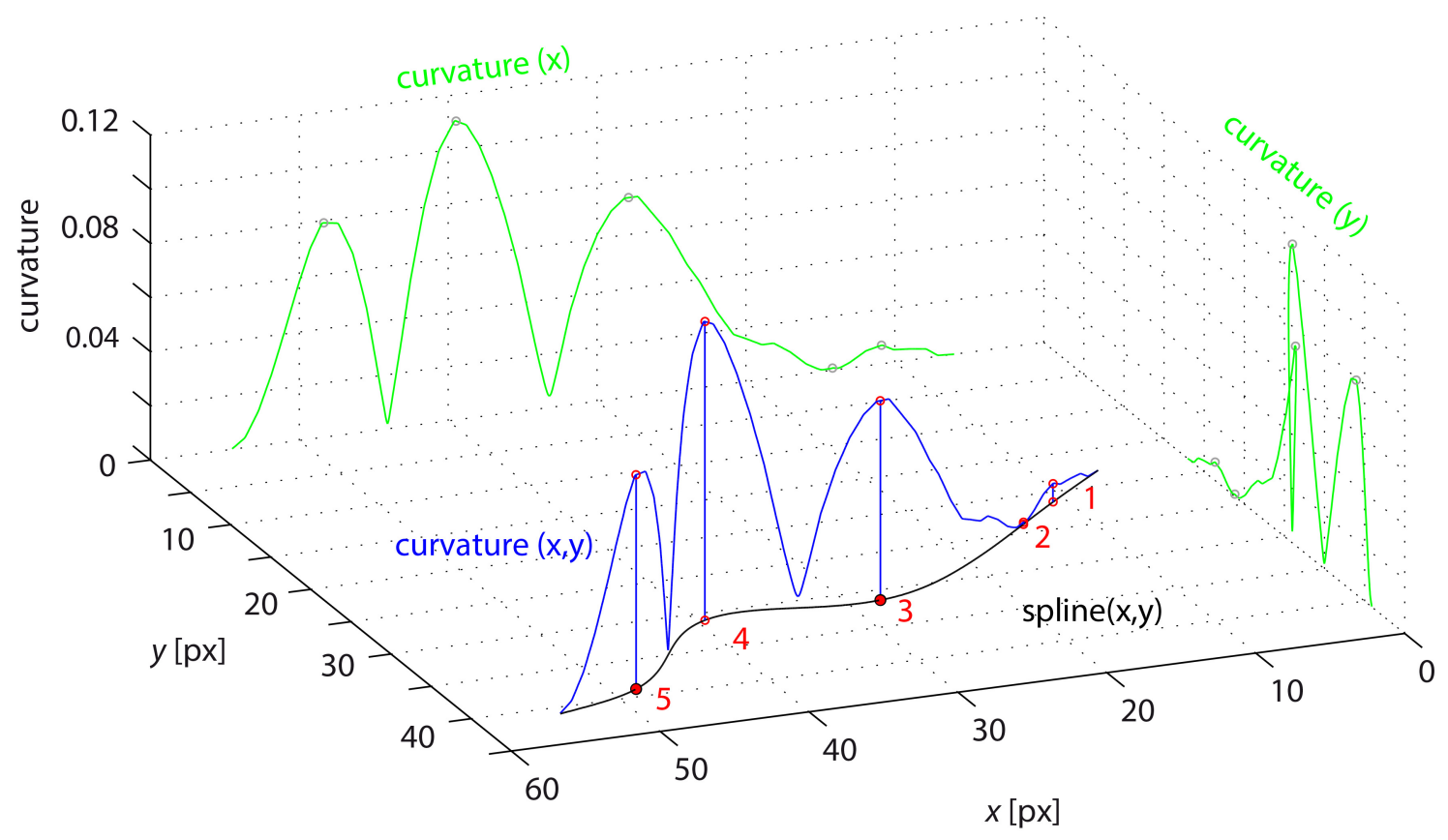

Figure 5.10: The black curve represents the spline fit to the original bundle. The curvature of this fit is shown in blue. To enhance the usability in case of more complex shapes, projections of the curvature onto the $x$ - and $y$-axis are also plotted in green. Local maxima are depicted with red dots and enumerated. The user has to manually chose the two extreme values which define the start and end points of buckling.

buckling waveform, the script subsequently highlights these and asks for a user selection of the peaks that delimit the region of deflection. At this point, the user has to decide whether the manually chosen parameter of the tolerance for fitting was suitable. The aim is to choose a tolerance, where the buckling is ideally detected by three peaks: One for the two ends of the buckling waveform and another which is in between, representing the maximal deflection. In the example depicted in figure 5.10 these would be peaks 3 to 5 . After the user has selected the two peaks representing the end points of the region of deflection, the Matlab script automatically calculates the distance in $\mu \mathrm{m}$ between the two points. Here, the pixel to $\mu \mathrm{m}$ conversion, which is needed for this step, is automatically carried out based on meta-data of the original confocal microscopy image. The distance obtained after unit conversion then constitutes the buckling wavelength, which is finally displayed together with the spline fit in an overlay with the microscopy image of the bundle (see figure 5.11 a). 


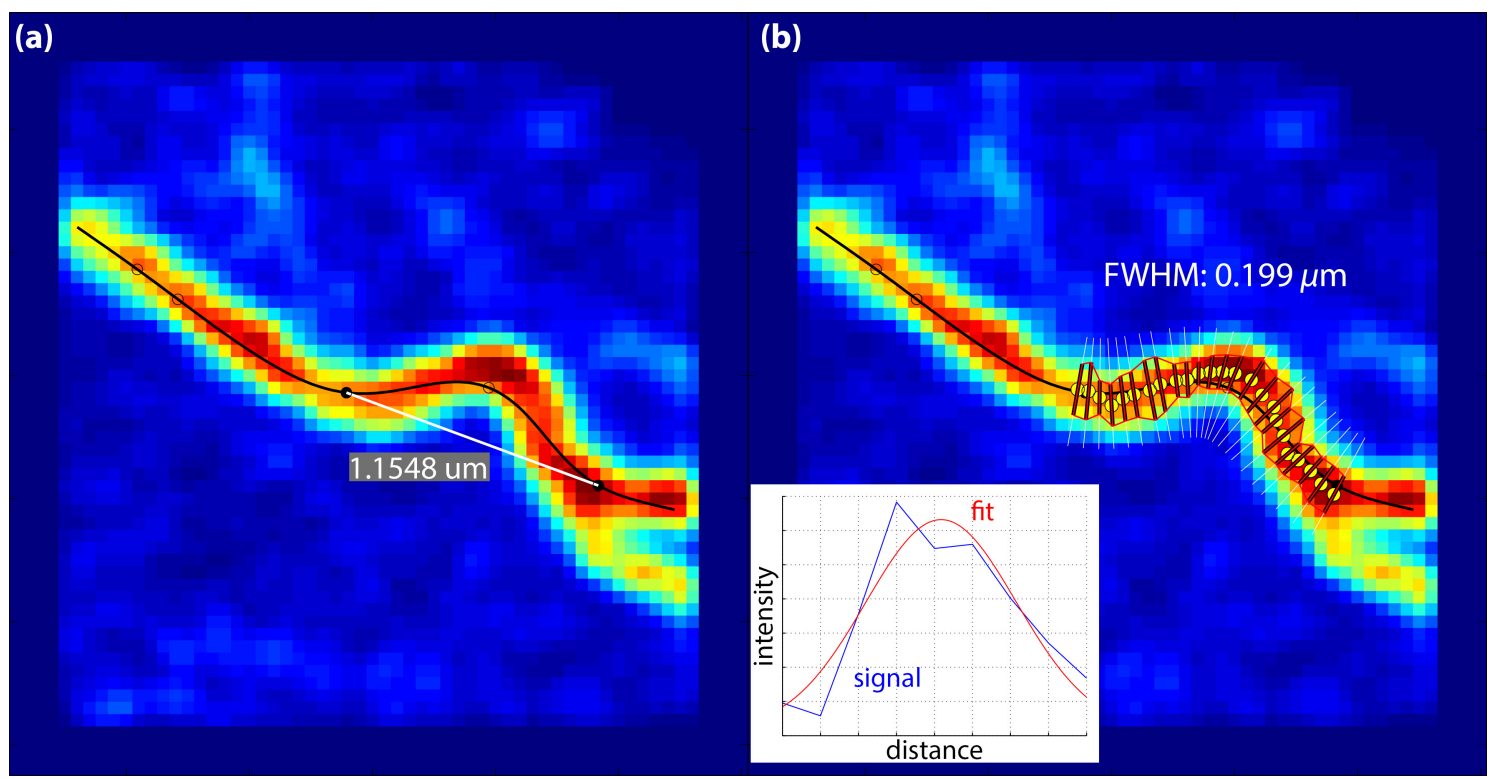

Figure 5.11: (a) The black curve shows the spline fit along a one-pixel-line which has been derived from the image shown. After calculating the second derivative of the curve, the buckling wavelength is determined as the distance between two maxima of curvature of the spline fit, which yields about $1.15 \mu \mathrm{m}$ for the example above. (b) A measure for the bundle thickness is determined by fitting Gaussian functions to intensity profiles along lines perpendicular to the spline fit (depicted as white lines). As an example, the intensity profile and the corresponding Gaussian fit for the first white line is shown in the inset. From the Gaussian fits the center position of the bundles (yellow circles) as well as the FWHM (red lines) are determined. The bundle thickness is then estimated from the median of all FWHMs calculated (about $0.2 \mu \mathrm{m}$ for the bundle shown).

\subsubsection{Analysis of the Bundle Diameter}

For later analysis, an estimate is required for the number of individual filaments inside each bundle. To be able to determine this value, we evaluate, at this stage, the thickness of the bundles in the fluorescence images. The thus obtained length $b_{\text {app }}$, however, still needs to be corrected for imaging effects due to fluorescence microscopy to represent the original bundle diameter. The steps involved in this correction are explained further on, while the extraction of $b_{\text {app }}$ from the images is described in the following paragraph.

To quantify the bundle thickness, we return to the spline fit of a bundle and draw lines perpendicular to the fit curve within the deflection region of the bundle. These lines are evenly distributed along the spline in intervals of about one pixel, and extend to a length of about $500 \mathrm{~nm}$. Their orientation is obtained at each point by rotating the respective tangent vector (first derivative) of the spline fit by $90^{\circ}$ using a corresponding rotation matrix. Afterwards, the intensity profiles are 
individually extracted along the lines. In a subsequent step, Gaussian functions (fit function: $a \exp \left(-\frac{(x-b)^{2}}{2 c^{2}}\right)+d$ ) with free parameters $a, b, c, d$ ) are fitted to the profiles and values for the full width at half maximum (FWHMs) are calculated $(2 \sqrt{2 \ln 2} c)$. Here, the fit parameters are initialized as follows: $a$ is approximated with the maximum of the intensity profile, $b$ is defined by the center of the line, $c$ is set to 10 , and $d$ is set approximately to the minimum of intensity within the image of the bundle i.e. the background signal (see figure $5.11 \mathrm{~b}$ ). The particular choice for $c$ is based on an estimate by eye of typical bundle thicknesses in the images and it was empirically found to give the fastest convergence of the fit. In a following step, the script calculates the median value of the FWHMs of all lines. The statistical value of the median is chosen since the Gaussian fits tend to lead to an overestimation of the bundle, e.g. in case of near network nodes, which would thus distort the mean value. The median is more robust against a bias introduced by these outliers.

As mentioned earlier, fluorescence images are not suitable for measuring the diameter of the original structure directly. The reason is that in imaging an object, the signal from the object is convoluted with a point spread function (PSF) to yield the detected signal. The PSF reflects image distortions resulting from diffraction and interference of the emitted electromagnetic waves (airy disks for point-sources), as well as aberrations caused by the microscope. An image can be represented by a superposition of many point sources where each is convoluted with the PSF. The whole detectable image $I$ is obtained by

$$
I(X, Y)=\iint I_{0}(x, y) \operatorname{PSF}(x, y ; X, Y) \mathrm{d} x \mathrm{~d} y,
$$

where $I_{0}$ describes the signal of the object, $x, y$ and $X, Y$ are coordinates of the object- and the image-plane, respectively (e.g. 126, chapter 11]).

Deducing the shape of the object from the recorded images would thus require prior knowledge of the PSF and even then the presence of random noise in the images signifies that there is no unambiguous solution to the deconvolution. That is, two slightly different noise patterns on the images can already lead to completely different results of the deconvolution. Such inversion problems are known as ill-posed problems and algorithms have been developed to yield stable and likely solutions (e.g. Lucy-Richardson deconvolution 127, 128]). However, measuring the PSF is already very challenging. It involves both the selection of well-characterized pointlike sources which ideally emit wavelengths which are identical to the sample, and the reproduction of the same imaging conditions that are also encountered during the experiments on the sample. In addition, the sample itself (i.e. the cell) is part of the optical setup and influences the PSF (e.g. [129, chapter 24]). Although 
blind deconvolution algorithms exist where the PSF is iteratively estimated by the algorithm itself, the restored image does not necessarily reproduce the correct size of the imaged object. We therefore opted for a more direct approach and chose to image beads of well-known diameters, which are comparable in size to the keratin bundles found in our cells. We assume the distortions which are caused by imaging to be similar for the two types of samples i.e. images of keratin bundles and beads and estimate the shift of a fitted FWHM to be the same for both objects.

To this end, we record green fluorescence beads with a diameter of $100 \mathrm{~nm}$ (FluoSpheres; Invitrogen, Darmstadt, Germany) which is about the same size as the diameter of keratin bundles 1 Using Matlab scripts, the center of the beads is determined by employing a circle finding algorithm [130]. Through the centers, four lines are defined with a changed orientation of $45^{\circ}$ each. We opted for this approach since the bundle orientation and thus also the direction of the pathways for the diameter determination is random. Furthermore, since the pixels are squares in shape the spatial resolution along the pixel diagonals is lower as compared to the resolution along horizontal or vertical lines. The intensity profile along each line is afterwards extracted and fitted with a Gaussian function (example shown in figure 5.12). In

${ }^{1}$ The size of the keratin bundles were assessed by SEM images, compare with figure 2.3 .
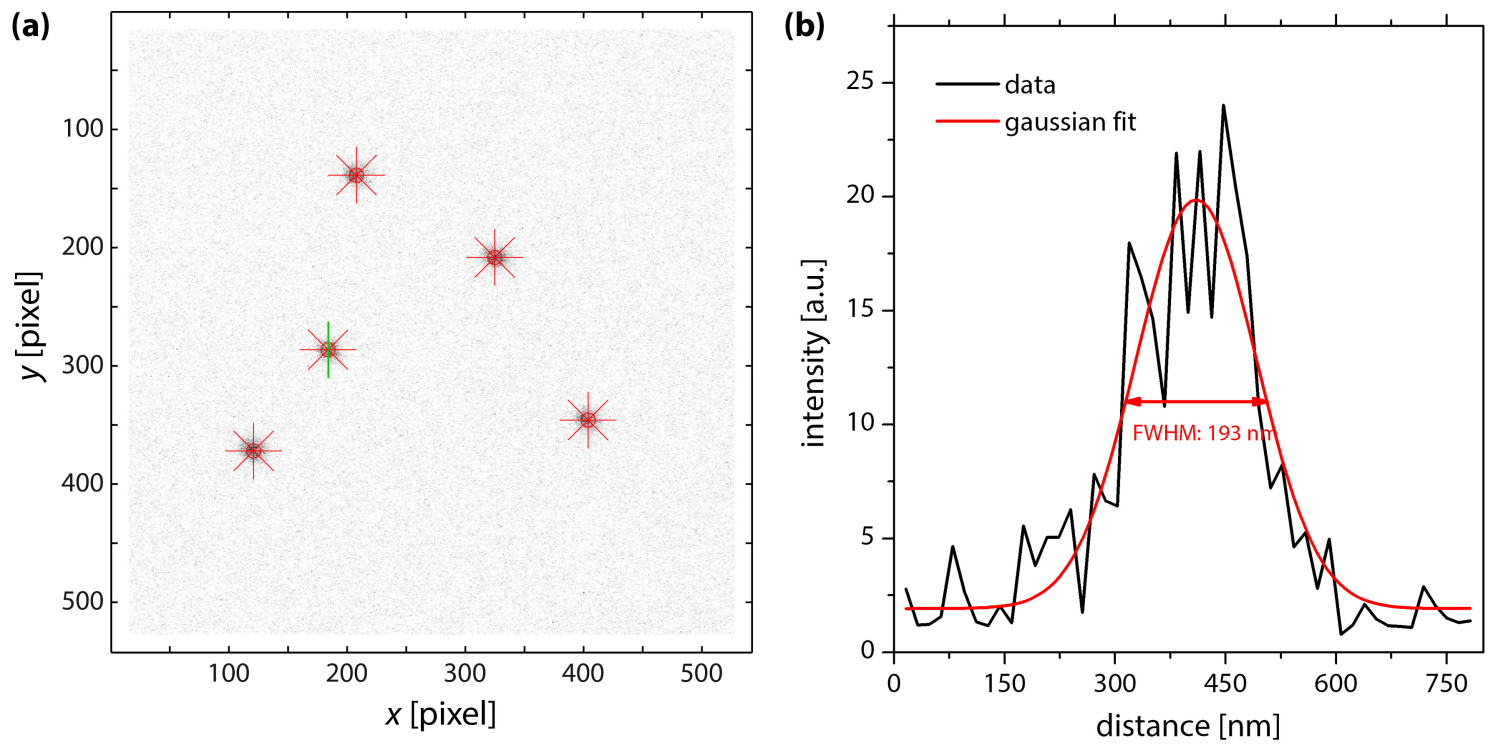

Figure 5.12: (a) Example of a confocal microscopy image of beads with a diameter of $100 \mathrm{~nm}$. The beads are detected using a circle-finding-algorithm with Matlab. Intensity profile along lines with four directions (see red lines) are subsequently extracted and fitted with an Gaussian function. (b) In black the intensity profile along the green line in image (a) is shown with the corresponding fit (red curve). On average, a FWHM of $188 \mathrm{~nm}$ is obtained. 
total, 487 beads have been evaluated with an average FWHM of $188 \mathrm{~nm}$. Thus, we conclude upon a shift! of $88 \mathrm{~nm}$ when comparing the calculated FWHM of a KIF bundle thickness with the real diameter.

\subsection{Results}

In the following, we first describe the organization of filaments within a bundle by presenting transmission electron microscopy (TEM) images. The determined bundle structure also constitutes the basis for the estimate of the number of filaments within a bundle of a certain diameter. Afterwards, the results from the evaluation of buckling events (i.e. taking the corresponding wavelength and the number of filaments into account) are presented.

\subsubsection{Structure of Keratin Bundles}

Based on the data of corrected bundle diameters (section 5.2.2), we are able to conclude upon the number of filaments in a bundle. To this end, both the lattice ordering and spacing of the individual filaments within a cross-section of a bundle have to be known.

Up to now, only little information is available about the ordering of the filaments inside keratin bundles in cells. In addition, the ordering alters depending on the cell type. In the stratum corneum, for example, which comprises the outermost layer of the skin, the keratin filaments are densely packed without leaving gaps. Norlén and Al-Amoudi have found a median inter-filament center-to-center distance for these cells of about $16 \mathrm{~nm}$ according to cryo TEM images of biopsied tissue, without specifying the type of keratin involved [131]. They propose a cubic-like rod packing (details given in 131,132$]$ ). The same authors have also revealed that the filament ordering is different within keratin bundles from viable cell layers of native vitreous epidermis. For these bundles, they have found a median filament center-to-center distance of about $11 \mathrm{~nm}$ and suggest hexagonal packaging of the KIFs.

The keratin filaments that we use for our investigations are composed of keratin K8 and K18 and have a diameter of about $10 \mathrm{~nm} \mathrm{[14].} \mathrm{To} \mathrm{investigate} \mathrm{the} \mathrm{organization}$ of individual KIFs inside a bundle we have looked at TEM images of our cells. These images were kindly provided by Dr. Wiebke Möbius. An example of a section along the long axis of the bundle and an example of a perpendicular cross-section are

\footnotetext{
${ }^{1}$ We use a shift for correction instead of a multiplicative factor, since the convolution with a $\mathrm{PSF}$ is a linear function according to the superposition principle (see equation 5.53 ).
} 

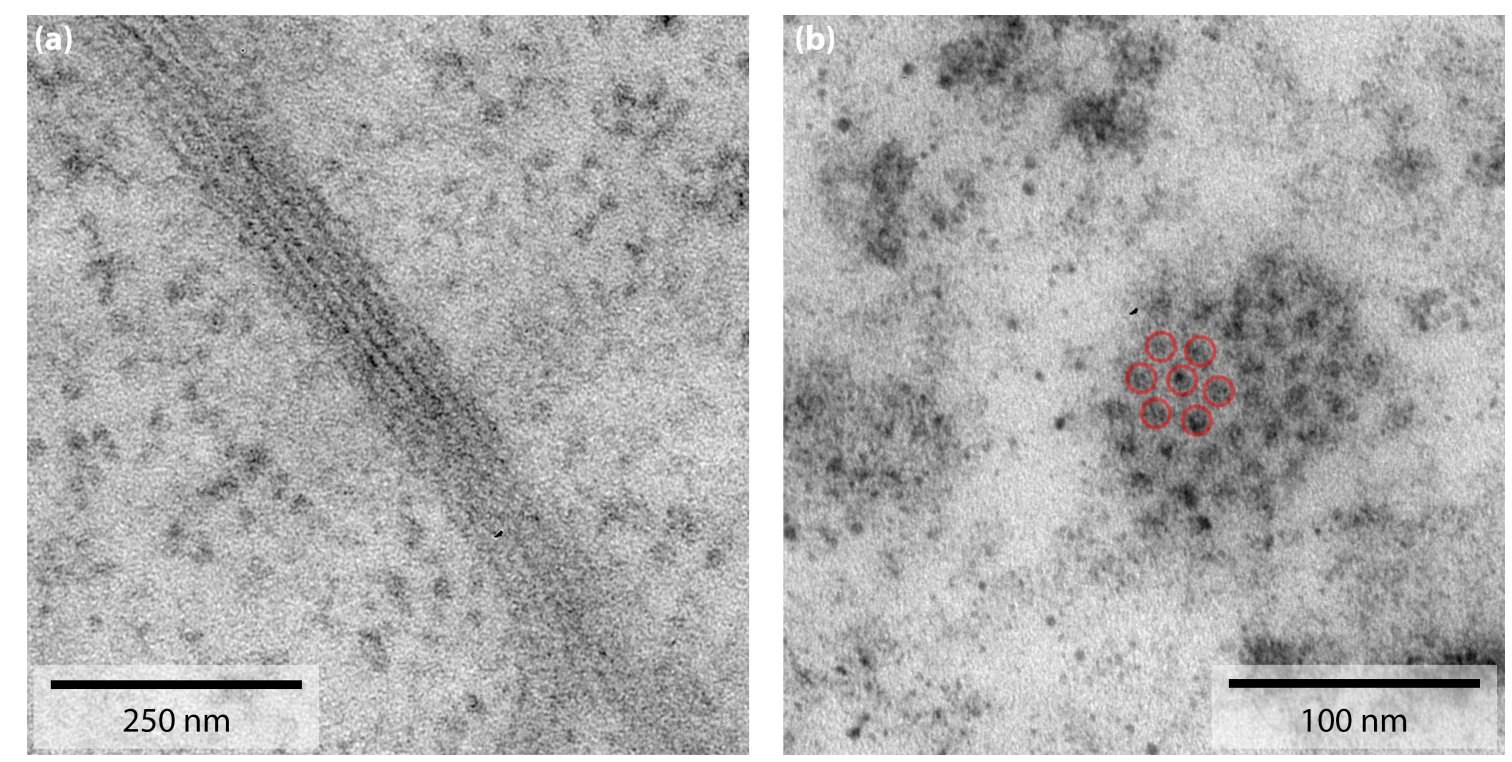

Figure 5.13: TEM images of keratin bundles in SK8/18-2 cells. (a) The average distance between the individual filaments is $12.2 \mathrm{~nm}$. (b) Cross-section of a KIF bundle showing a hexagonal lattice ordering (indicated by red circles). Images were provided by courtesy of Dr. Wiebke Möbius.

shown in figure 5.13 .

From the TEM sections along the long axis of the bundle, we deduce an average distance of about $12.2 \mathrm{~nm}$ between individual filaments (based on the analysis of four bundles). Since the precise orientation of the bundle within the TEM sections is unknown, this value is expected to overestimate the real distance: The actual distance of nearest neighbors $d_{\mathrm{NN}}$ is only directly visible in a TEM section if the section is cut through the bundle along a line connecting nearest neighbors (for a hexagonal lattice this is the case for angles of $n \cdot 60^{\circ}$ with $n \in \mathbb{N}$, see also figure 5.14). In all other instances, the TEM sections show inter-filament distances that are larger than $d_{\mathrm{NN}}$. To further analyze the distances and also conclude upon the filament organization within a bundle, we turn to the TEM images of cross-sections perpendicular to the bundle axis. As shown in figure $5.13 \mathrm{~b}$ a basic hexagonal ordering of the individual KIFs can be confirmed for K8K18 keratin bundles. Here as well, the orientation is crucial when measuring the distance between filaments, which are visible as dark spots. It needs to be considered, however, that a dislocation of the filaments and a possible increase in inter-filament distances due to sample preparation for TEM imaging cannot be excluded. This point receives considerable weight when taking into account that the bundling behavior is most likely a result of electrostatic and ion-mediated interactions (compare with discussion in section 5.4.2) which may be influenced during the staining process. 


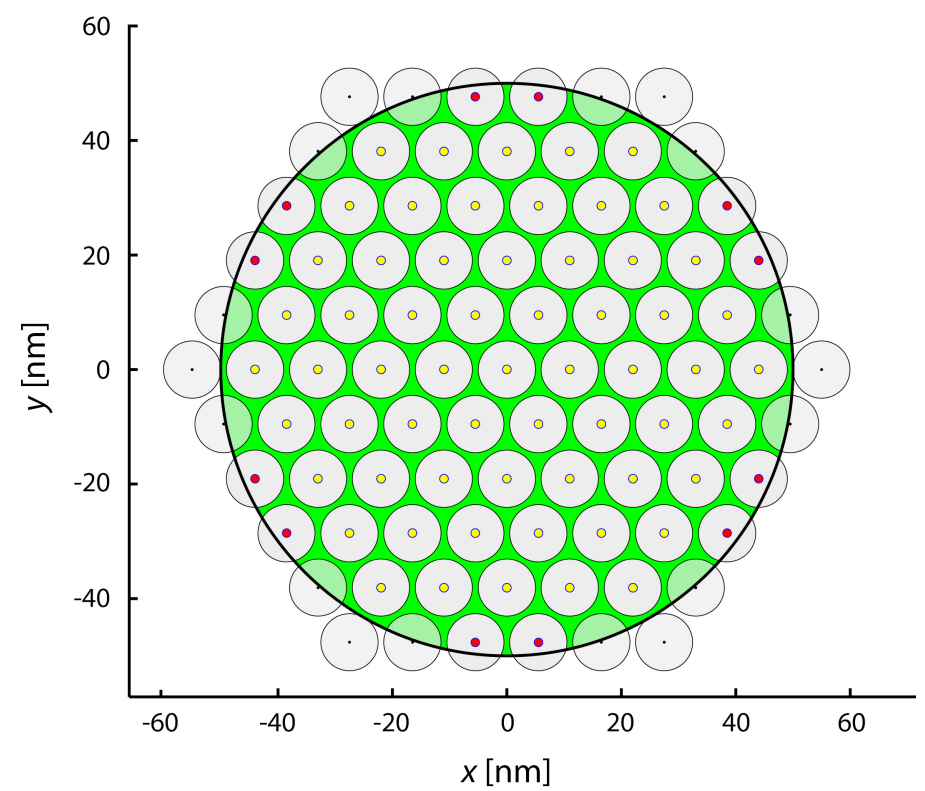

Figure 5.14: Numeric approach to determine the number of filaments inside a bundle with a certain diameter. Here, a bundle with a thickness of $100 \mathrm{~nm}$ (green) consists of 73 individual filaments (gray) which are completely (yellow centers) or mostly (magenta centers) inside the large circle. The filaments have each a diameter of $10 \mathrm{~nm}$ and a centerto-center distance of $11 \mathrm{~nm}$.

Based on our data from the TEM images and literature 131,132, we assume the KIFs inside a bundle to be in a hexagonal lattice with a center-to-center distance of $11 \mathrm{~nm}$. Using a self-written Matlab script to model the bundles, we are thus able to conclude upon the number of filaments in a bundle with a certain diameter assuming a cylindrical shape of the bundle. Figure 5.14 shows an example of a model bundle which has a thickness of $100 \mathrm{~nm}$. All filaments whose areas are included by more than $50 \%$ in the circle which represents the bundle border are counted as constituents of the bundle. For this illustration, the center $(0,0)$ of the bundle was set to coincide with the position of a filament in the bundle. As might be expected, the question of whether the center of a bundle lies on top of a filament or in between filaments has an influence on the determined $N$ of filaments inside a bundle of a given diameter. To take this influence into account, the number of filaments is calculated for both cases and the value of $N$ is then determined by taking the average of the two calculations 1 .

\footnotetext{
${ }^{1}$ The two configurations, where the center of the lattice is on top of a filament or exactly in between filaments, represent extreme cases. By averaging these two cases we cover any configuration possible for the arrangement of filaments inside a bundle.
} 


\subsubsection{Buckling of Keratin Bundles}

As described in section 5.1, the wavelength of buckling events for keratin bundles in SK8/18-2 cells is determined by basically four parameters: The properties of the individual keratin filaments which comprise a bundle (i.e. their persistence length $L_{\mathrm{PF}}$ and their number $N$ within the bundle), the strength of coupling between the filaments (i.e. the coupling parameter $\gamma$ ) and the elastic properties of the surrounding medium (expressed by the parameter $\alpha$ ):

$$
\lambda=2 \pi\left(\frac{N^{\gamma} \cdot L_{\mathrm{PF}} \cdot k_{\mathrm{B}} T}{\alpha}\right)^{1 / 4} \quad, \gamma \in[1,2]
$$

This equation can also be rewritten as

$$
\begin{aligned}
\ln \left(\lambda^{*}\right) & =\frac{1}{4} \gamma \ln (N)+\text { const } \\
\text { const } & =\frac{1}{4} \ln \left(L_{P F}^{*} \cdot k_{B}^{*} T^{*}\right)+\ln (2 \pi)-\frac{1}{4} \ln \left(\alpha^{*}\right), \quad \alpha^{*}=\frac{4 \pi}{\ln (l / b)} G^{*} .
\end{aligned}
$$

Here, the parameters marked with a star indicate dimensionless parameters 1, Of the unknown parameters which appear in equation (5.54), we measure the buckling wavelength $\lambda$ directly (see chapter 5.2 and calculate the number $N$ of filaments

${ }^{1}$ e.g. $\lambda^{*}=\lambda / \lambda_{0}, \lambda_{0}=1 \mathrm{~m}$
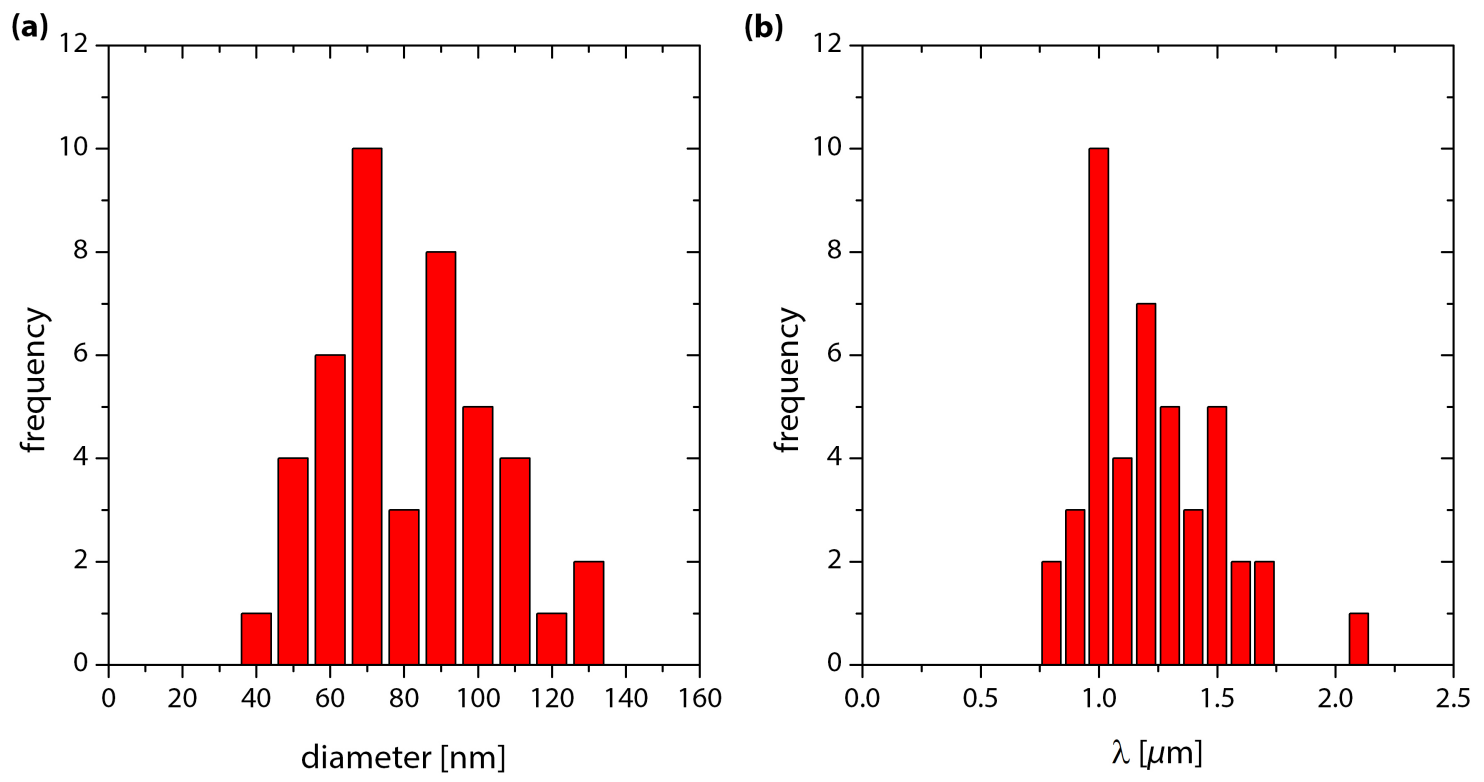

Figure 5.15: Histogram of KIF bundle diameters extracted for buckling events in live SK8/18-2 cells (a), and the corresponding buckling wavelength distribution (b). 
in a bundle based on the experimental data of the bundle diameters (according to chapter 5.3.1). In total, 44 buckling events within 16 cells have been analyzed. The recorded data contain bundles with diameters from about 40 to $130 \mathrm{~nm}$ (on average $81 \mathrm{~nm}$ ) which show buckling events with wavelengths between 0.8 and $2.1 \mu \mathrm{m}$ (on average $1.2 \mu \mathrm{m}$ ). An overview of the results is given in figure 5.15, where histograms of the determined bundle diameters and buckling wavelengths are depicted.

The number of filaments per bundle cross-section $N$ is then plotted on a logarithmic scale against the respective buckling wavelength (figure 5.16). Here, the logarithmic expression is particularly useful for plotting the data, since the coupling parameter $\gamma$ is thus separated, according to equation (5.55), from the other variables. In fact, the term $\gamma / 4$ describes the slope of the now linear function directly and the remaining parameters in equation (5.55) are expressed in terms of the intercept. To estimate an experimental error for $\ln (N)$ in the plot in figure 5.16, we have assumed that the error which was introduced by the determination of the bundle diameters

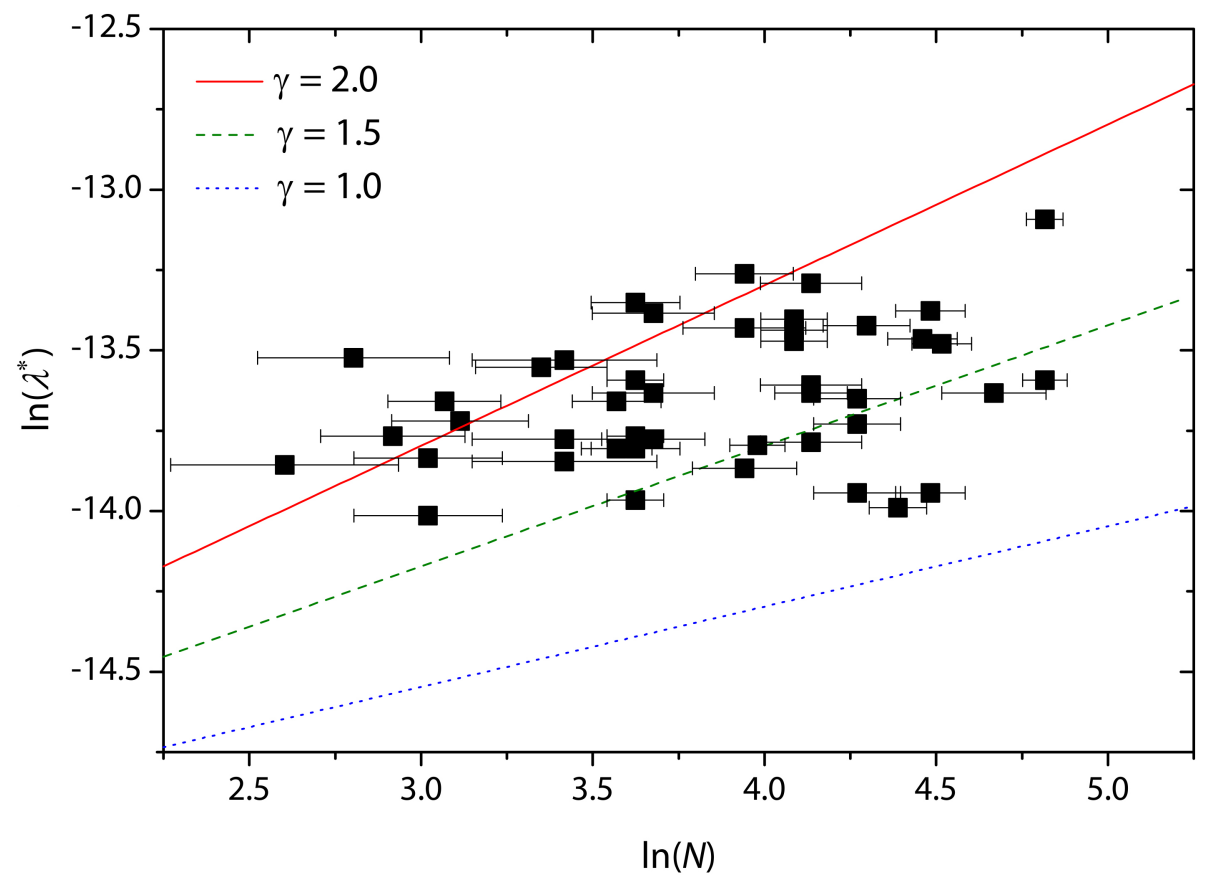

Figure 5.16: Buckling wavelength $\lambda$ plotted against the number $N$ of filaments in a bundle. In addition, three lines for different coupling parameters $\gamma$ are depicted showing the upper and lower theoretical limits for coupling as well as an intermediate state. Their intercepts with the $y$-axis are defined by the persistence length of one filament, which is here set to $L_{\mathrm{PF}}=780 \mathrm{~nm}$, and the elastic modulus of the surrounding matrix, which is assumed to be $E_{\mathrm{SM}}=1.5 \mathrm{kPa}$. As $N$ is calculated from experimentally determined bundle diameters, the error bars for $N$ are obtained from error estimates of $\pm 5 \mathrm{~nm}$ for the diameters. 
is $5 \mathrm{~nm}$. The corresponding error bars in terms of $N$ have then been obtained by recalculating $N$ for a diameter that was increased and decreased by $5 \mathrm{~nm}$, respectively. As before, we have considered for each recalculation of $N$ two geometries for hexagonally ordered bundles: one for which a filament is positioned at the center of the bundle and the other for which the distance of the bundle center to the next constituents of the bundle is maximal. The results for the two geometries have been averaged for each recalculation of $N$ to yield $N_{+5 \mathrm{~nm}}$ and $N_{-5 \mathrm{~nm}}$. The error for $N$ is then estimated by calculating the differences between the original value for $N$ and the two variants $N_{+5 \mathrm{~nm}}$ and $N_{-5 \mathrm{~nm}}$. The error bars shown in figure 5.16 thus represent the maximal variance.

In addition to our data points, figure 5.16 also contains three lines that were added to set the experimental data into perspective. Here, the red and blue lines represent theoretical limits for the maximum and minimum coupling parameters possible ( $\gamma=2$ and $\gamma=1$ respectively), and the green curve shows an intermediate state of coupling $(\gamma=1.5)$. The intercepts of these three lines depend on the following (see equation (5.56): the persistence length of an individual filament $L_{\mathrm{PF}}$, the shear modulus of the surrounding matrix $G$ and characteristic lengths scales $l$ and $b$ for the buckling events. Here, $l$ represents a typical size of the filament segment that moves during buckling (compare with section 5.1.3) and we have consequently inserted a value for $l$ which is on the order of the buckling wavelength $(1 \mu \mathrm{m})$. In addition, we substitute the parameter $b$ by the mean diameter of KIF bundles $\left(81 \mathrm{~nm}\right.$ ), while assuming $L_{\mathrm{PF}}$ to be about $0.8 \mu \mathrm{m}$ (for an in-detail description of the chosen value of $L_{\mathrm{PF}}$ and the influence of these parameters, see section 5.4.1 in the discussion). As described in section 5.1.3, the shear modulus can be expressed in terms of the elastic modulus by $G=\frac{E}{2(1+\nu)}$, where $\nu$ describes the Poisson ratio. In agreement with reference [133, we assume $\nu=0.5$ (volume remains constant) and for the elastic modulus of the cytoplasm $E_{\mathrm{SM}}=1.5 \mathrm{kPa}$ which leads to $G=0.5 \mathrm{kPa}$.

As depicted in figure 5.16 about $80 \%$ of our data points lie above the green line that corresponds to an intermediate state of coupling between bundle constituents. Hence, most of our data points indicate a coupling parameter above 1.5. This result suggests a strong coupling between individual filaments within the KIF bundles. Furthermore, we see that the data points do not follow a straight line over the whole range of $N$. An initial tendency for strong coupling ( $\gamma$ near 2) within thin bundles becomes less pronounced with increasing bundle diameter. For bundles which contain more than $\sim 50$ filaments $(\ln (50) \approx 3.91)$ the data points are more broadly spread, and for $N>70(\ln (70) \approx 4.25)$ the data points suggest a lower coupling parameter. 


\subsection{Discussion}

\subsubsection{Influence of the Surrounding Matrix}

As mentioned previously, the main factors defining the intercepts of the curves in figure 5.16 are basically the persistence length of an individual filament $L_{\mathrm{PF}}$ and the shear modulus $G$ of the medium surrounding the bundle. Any uncertainty in the intercept must therefore relate to sources of errors in $L_{\mathrm{PF}}$ and $G$. In the following, these sources of errors are discussed in detail for each of the two parameters and the consequences for intra-bundle coupling are described.

The value of $L_{\mathrm{PF}}$ that we have used for our analysis has been adapted from findings by Lichtenstern et al. [14]. By investigating atomic force microscopy (AFM) and EM images of K8K18 KIFs, Lichtenstern et al. have concluded from their in vitro experiments that $L_{\mathrm{PF}}$ is in the range between 300 and $480 \mathrm{~nm}$. Notably, the authors have also found that the persistence length depends on the ionic environment, a factor which is difficult to control in cells. Moreover, the persistence length for adsorbed filaments using EM or AFM tends to be underestimated compared to

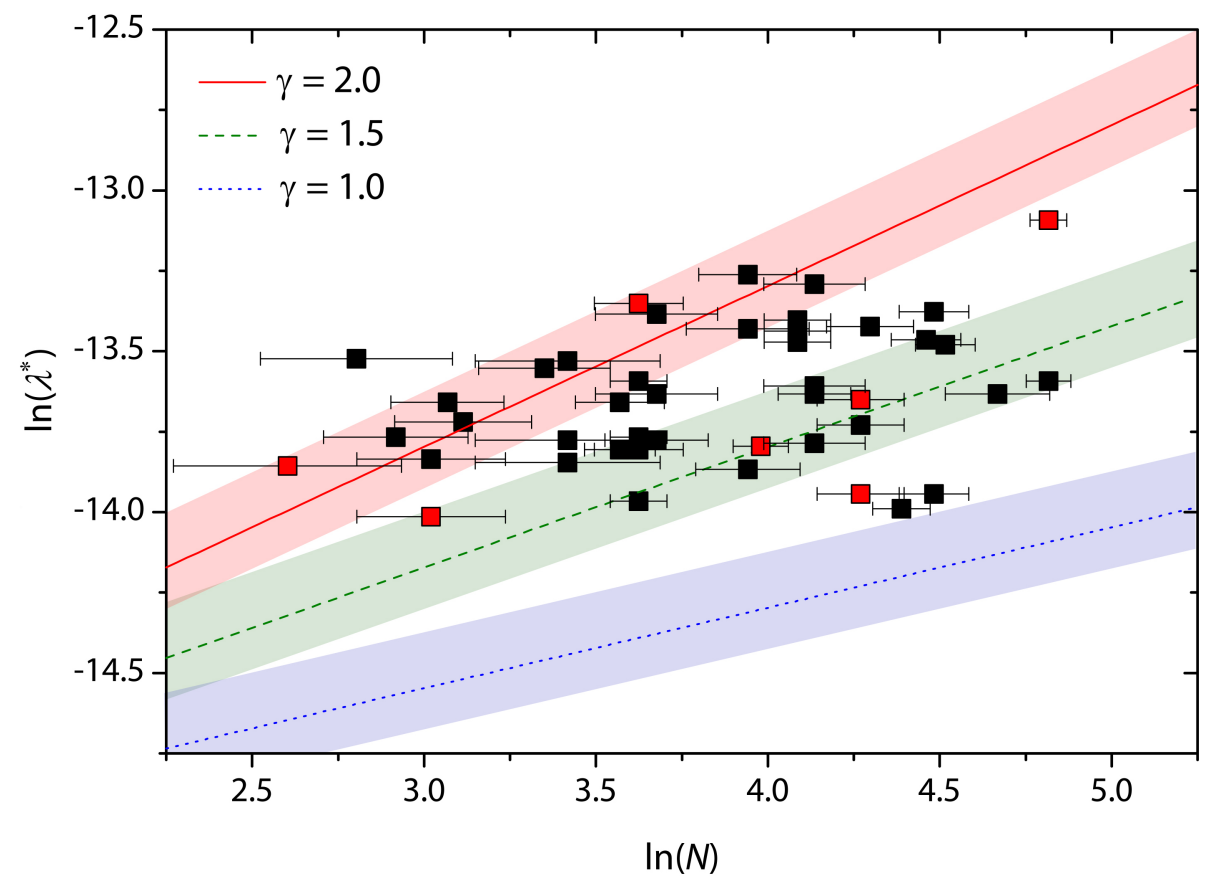

Figure 5.17: Buckling wavelength $\lambda$ plotted against the number $N$ of filaments in a bundle as shown in figure 5.16. Here, also an error range for the intercept is included (depicted as shaded, color-coded regions) which mirrors possible variations in the elastic modulus of the surrounding matrix $\left(E_{\mathrm{SM}} \in[750,2500]\right)$. In addition, data points which belong to buckling events at the cell periphery are shown in red. 
the actual persistence length in solution, due to filament-substrate interactions. For instance, it has been shown that a freely fluctuating vimentin filament has an actual persistence length which is twice as high as the value measured in static experiments on filaments that adhere to a surface [134]. Unfortunately, there are no data of freely fluctuating K8K18 KIFs available yet. Consequently, we need to rely on plausible assumptions to obtain the actual persistence length of K8K18 filaments. Following the findings which are described in reference [134], we assume an equilibrium persistence length for K8K18 filaments which is twice as high as the average measured by Lichtenstern and colleagues, i.e. we estimate $L_{\mathrm{PF}}=0.78 \mu \mathrm{m}$.

Further studies, in which the persistence length of a freely fluctuating K8K18 keratin filaments is determined, are necessary to more precisely assess the coupling within K8K18 bundles. Yet, if the true value for $L_{\mathrm{PF}}$ turned out to be smaller than our estimation of $0.78 \mu \mathrm{m}$, the straight lines in figure 5.17 would move downwards. Under such conditions, our finding of a strong coupling (see section 5.4.2) would be even more emphasized.

The second parameter which influences the intercept in figure 5.16 is the shear modulus $G$ of the bundle environment. Here, we have calculated $G$ on the basis of the elastic modulus $E_{\mathrm{SM}}$ of the cytoplasm, which is assumed to be $1.5 \mathrm{kPa}$ corresponding to typical values in cells (e.g. [133]). However, the mechanical properties are highly inhomogeneous within a cell. Local differences in the elastic modulus occur due to a nonuniform distribution of the cytoskeleton (e.g. dense areas in the cytoplasmic actin network or actin stress fibers) and the organelles, or due to the presence of the nucleus. Indeed, Rother and co-workers have found distinct differences in the elastic modulus when probing SK8/18-2 cells with AFM. Their findings for $E_{\mathrm{SM}}$ range from $100 \mathrm{~Pa}$ up to several $1000 \mathrm{~Pa}$ leading to a median value of about $1500 \mathrm{~Pa}$ 135. When interpreting our data, it is therefore important to keep in mind that the exact elastic modulus for each data point in figure 5.17 is unknown and may vary.

Furthermore, we have converted the elastic modulus into the shear modulus for our analysis under the assumption that the Poisson's ratio is 0.5 (incompressible material, where the volume is constant during deformation) for cells according to literature $(e . g .[133)$. It is, however, debatable whether this estimate is correct for every buckling event. While fluids are mostly incompressible, polymers may behave differently. As a consequence, the local Poisson's ratio in a cell may vary e.g. due to a local dominance of the actin response (in vitro actin networks have a Poisson ratio of about $0.4[136]$ ). For values of $\nu$ that are smaller than 0.5 , our finding of a primarily strong coupling between the KIFs inside a bundle is, again, enhanced. 


\subsubsection{Strong Coupling between Filaments inside a Bundle}

Most of our data suggest a strong coupling between the individual KIFs inside a bundle (section 5.3.2). In fact, 14 out of 44 buckling events imply a coupling parameter $\gamma$ of 2 according to the area shaded in red in figure 5.17, which represents a tolerance region for variations in the elastic modulus of the cytoplasm. Furthermore, 41 of 44 data points indicate that $\gamma$ is around or above 1.5. As described in the theory (section 5.1.5), a coupling parameter of 2 describes a bundle in which its constituents are not allowed to slide along each other, while a value of 1 means a completely free axial movement of the individual filaments. Hence, we have found great evidence for mostly strong links between filaments inside a bundle. Consequently, an axial sliding of KIFs is possible, but rather restricted.

The importance of the coupling strength for the persistence length of a bundle can be assessed when looking at figure 5.18, where the persistence length of the bundle $L_{\mathrm{PB}}$ is depicted as a function of $\gamma$. As $L_{\mathrm{PB}}$ is given by

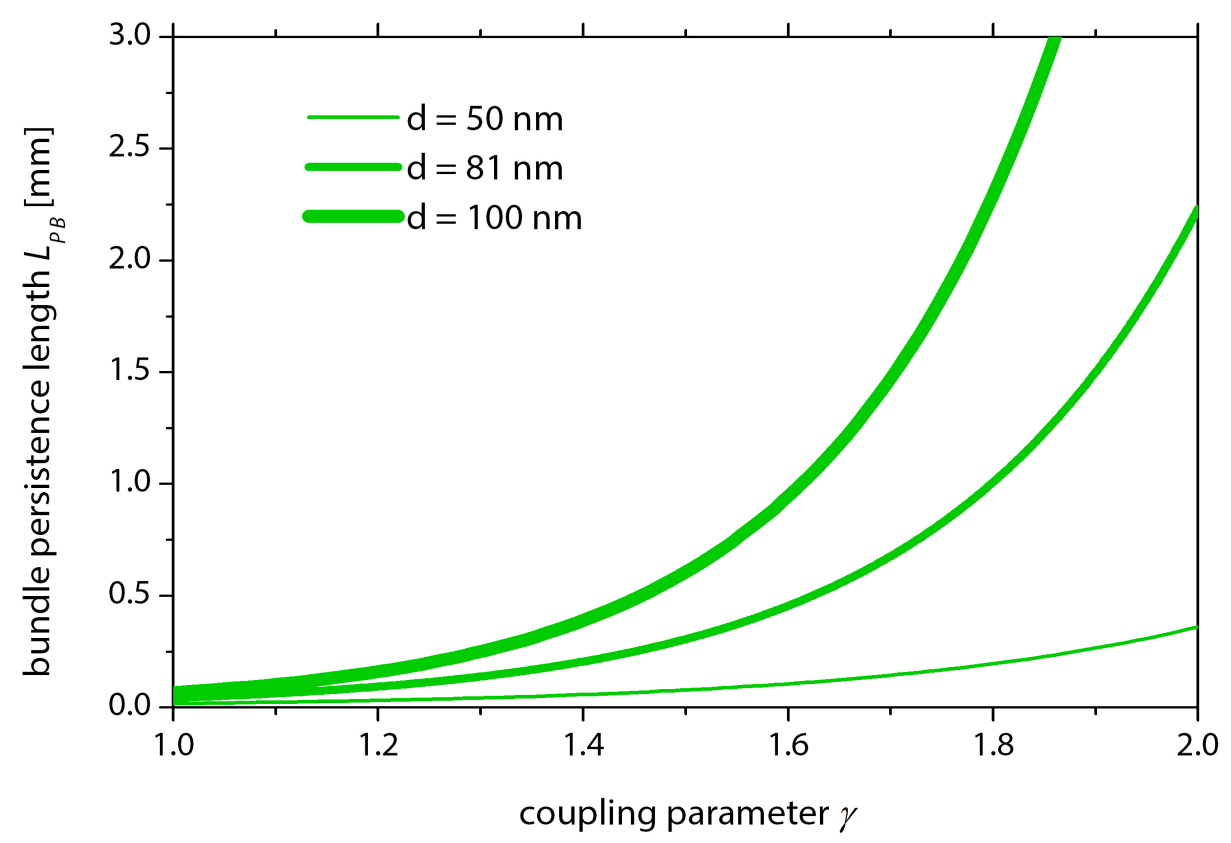

Figure 5.18: Persistence length $L_{\mathrm{PB}}$ of a bundle as a function of the coupling parameter $\gamma$. The curves shown are calculated based on the persistence length $L_{\mathrm{PF}}=0.78 \mu \mathrm{m}$ of a single filament and the total number $N$ of filaments within a bundle of a diameter of 50 , 81 or $100 \mathrm{~nm}$, respectively. Moreover, the filaments within a bundle are assumed to be arranged on a hexagonal lattice with a lattice constant of $11 \mathrm{~nm}$. Since $L_{\mathrm{PB}}$ follows an exponential increase $\left(L_{\mathrm{PB}}=N^{\gamma} \cdot L_{\mathrm{PF}}\right.$, see section 5.1.5), the persistence length is in the range of $\mathrm{mm}$ for strong coupling within an average bundle of a diameter of $81 \mathrm{~nm}$. 


$$
L_{\mathrm{PB}}=N^{\gamma} \cdot L_{\mathrm{PF}}, \quad \gamma \in[1,2]
$$

$\gamma$ has a marked influence on the persistence length (see also section 5.1.5). Assuming a keratin filament persistence length of about $0.8 \mu \mathrm{m}$, an average bundle with a diameter of $81 \mathrm{~nm}$ shows a pronounced increase of its stiffness with increasing $\gamma$. Thus, when the filaments are arranged together without inter-linkage, the bundle has a persistence length of about $0.04 \mathrm{~mm}(\gamma=1)$. By introducing coupling between the KIFs, $L_{\mathrm{PB}}$ increases strongly to $\sim 0.3 \mathrm{~mm}(\gamma=1.5)$ or even $\sim 2.2 \mathrm{~mm}$ in case of maximal coupling $(\gamma=2)$. Interestingly, the value for $L_{\mathrm{PB}}$ for maximal coupling in keratin bundles is of the same magnitude as the persistence length of microtubules $(\sim 5 \mathrm{~mm}[137])$. As the persistence lengths for the two systems are comparable, so are their buckling wavelengths. Indeed, Brangwynne et al. identified wavelengths of about $3 \mu \mathrm{m}$ which is in good accordance with our finding of $\lambda \sim 1 \mu \mathrm{m}$. The large persistence length of keratin bundles, in turn, signifies that a network built up of strongly inter-linked KIF bundles provides the cell with great mechanical stability. Recent studies support this point further (see also section 2.2.3): By measuring the elastic modulus of keratin-deficient cells in comparison to wild-type cells, Ramms et al. have found that keratinocytes are about $40 \%$ softer [48], when a keratin network is lacking. Furthermore, cells without keratin have a deformability which is $60 \%$ higher in comparison to wildtype cells [49].

As the data in figure 5.17 show, in larger bundles the coupling seems to be weaker than in bundles with smaller diameters. However, the data only provide information about the average coupling in a bundle. Considering a bundle in vivo, an inhomogeneous linkage between individual KIFs within a bundle would be conceivable, since the keratin structure undergoes continuous changes (see [52 54] and also section 2.2.3). To explain this point further, we need to return to the bundle building process. As described previously, bundle formation can be initiated in vitro by the addition of monovalent or multivalent ions to a filament suspension (see section 2.2.1 for details). Under such conditions, the aggregation of filaments proceeds without further restrictions by e.g. proteins, which may finally lead to a concentration of all IFs to one region (e.g. [18]). In cells, however, proteins are involved in the bundling process: They moderate the cross-linking of filaments (e.g. filaggrin) and regulate bundle formation (e.g. epiplakin). The regulatory function of proteins may, moreover, influence the strength of coupling within a bundle: Since proteins seem to limit the thickness of a bundle, it is conceivable that the outer filaments within a bundle are bound less tightly to each other than filaments which are located near the center. Taking this hypothesis into account, the lower average 
coupling factor for thicker bundles appears plausible. Another possible explanation for the weaker coupling in thicker bundles would be that larger bundles form, due to a collapse of smaller ones. Indeed, the TEM image in figure 5.19 shows such a merging of two bundles. As a consequence, only few keratin filaments are involved in the establishment of links at the contact point between the original two bundles, which in turn leads to a weaker total interconnectivity.

Our finding of a relatively strong linkage between individual filaments in the majority of KIF bundles is most likely a consequence of a combination of both keratin associated proteins and ionic conditions. Consequently, the bundles and thus also the keratin network build stiff structures, which can be considered as backbones of the cell. This circumstance may be especially relevant to cells under conditions, where actin stress fiber formation is less pronounced and filamentous actin is only present as a gel-like network (e.g. cells on softer substrates [114]). Further studies about the influence of actin on buckling events, particularly with regard to actin stress fibers, may contribute to a deeper understanding of the interaction between keratin and actin. Here, one question that would be interesting to address concerns whether or not the observed buckling events are co-localized with actin stress fibers.

Since stress fibers are mostly located at the cell periphery (compare with figure

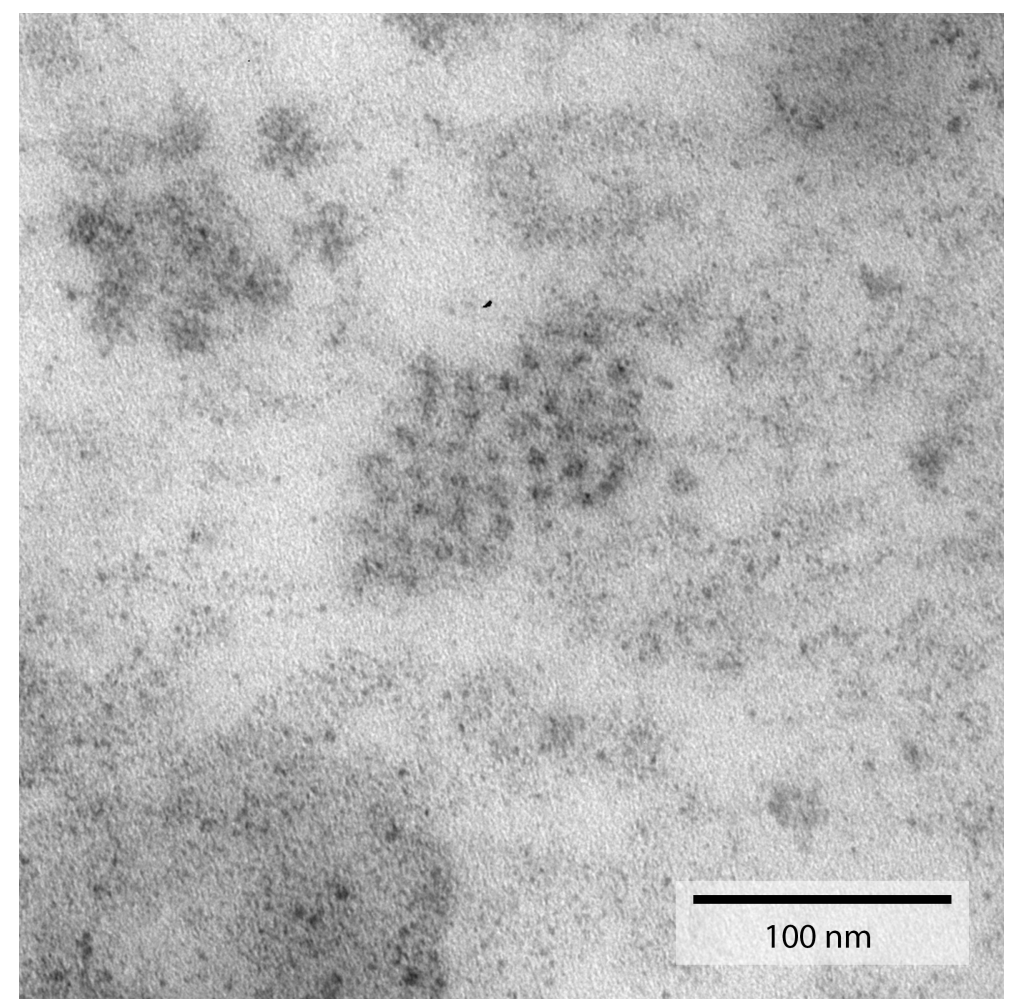

Figure 5.19: TEM image of a keratin K8K18 filament bundle composed of two subbundles. Image was provided by courtesy of Dr. Wiebke Möbius. 
2.1 and 4.17), a special analysis of buckling events in this region may lead to further fruitful insights. Apart from possible interactions of keratin with stress fibers, there is yet another reason why an investigation of buckling events at the cell periphery would be appealing, which is linked to the keratin cycle. As described in section 2.2.3 during the keratin cycle keratin particles are build at the cell periphery and are included into the preexisting keratin network during their transport along actin fibers towards the nucleus. By investigating buckling events of keratin bundles at different positions in the cell, the properties of newly formed bundles could be revealed. In particular, the linkage between freshly arranged filaments may be not as tight yet as the connections of longer established bundles. During our study, we have focused on the region below the nucleus, where the keratin network is very persistent and thus provides good comparability throughout the whole cell population. Nevertheless, 7 out of our 44 data points were extracted from buckling events near the cell periphery (red squares in figure 5.17). So far, we have not been able to determine a unique behavior for these buckling events, which could be simply a result of the lack of adequate statistics. The main challenge which an observation of bundles at the cell periphery entails is the correct identification of buckling events. Since the network is less pronounced, only few nodes restrict the bundle motion and can thus serve as a reference to differentiate between waveforms induced by compressive forces (i.e. buckling) and shapes that are caused by transverse pulling forces.

Our knowledge of the coupling properties between individual KIFs in a bundle could be increased in two ways. First, we suggest the execution of further studies on buckling events in cells. Here, the knockout of different proteins (e.g. filaggrin or members of the plakin protein family) may lead to a deeper understanding of the proteins' function, in particular with regard to their role for bundling. A potential change of the observable buckling wavelengths in knockout cells would indicate an alteration in the coupling strength between individual KIFs inside a bundle. Thus, such experiments would shed light on the origin of the relatively high coupling parameter which we have identified in our study. Secondly, we propose to complement the in vivo studies with an in vitro approach. Since in vitro experiments allow for very controlled environmental conditions, it would be possible to determine the influence of different ion concentration on the strength of the coupling inside a bundle.

\subsubsection{Theoretical Model}

For our analysis of the buckling behavior of keratin bundles in living cells, we have focused on the model of Brangwynne et al. [73]. This model assumes that constrained buckling is defined by the elastic contribution of the buckled rod, a linear elastic 
contribution resulting from the transverse displacement of the surrounding matrix, and a contribution from the compressive forces which cause the buckling event. As described in section 2.3, the model by Brangwynne et al. has been extended by two groups to include first order non-linearities in the elastic response of the surrounding matrix and longitudinal coupling between the rod and its environment [79, 80]. Interestingly, both the critical force which is required to induce a buckling event, as well as the resulting buckling wavelength $\lambda$, are unaffected by these extensions of the theory. However, both research groups have found that the amplitude is altered in their models, which finally leads to a waveform with an overlaid exponential decay.

Since we observe only a single wave peak and we cannot confirm an exponential decay of the buckling waveform within our data, we can neglect both nonlinear elastic properties and longitudinal deformations of the surrounding medium. This is especially valid, because we are solely interested in analyzing the buckling wavelength itself, which is, as described earlier, unaffected by these terms.

In our analysis, we furthermore treat the environment as an elastic material and disregard viscous effects. This neglect of viscous properties of the surrounding matrix is justified by the fact that viscosity solely affects the dynamics of buckling (e.g. the growth rate), but has no influence on the buckling shape itself [81, 82]. Accordingly, the model of Brangwynne et al. is applicable to our data of buckling in keratin structures. We have nevertheless modified it by introducing the interfilament coupling parameter, to take the bundle character of our buckled rods into account (section 5.1.5).

\subsubsection{Buckling in Sheared Cells}

Bridging our two experimental blocks on the cellular response to shear stress and on the mechanics of KIF buckling events, we return to our data of cells under shear flow conditions (see chapter 4) and analyze naturally occurring buckling of keratin bundles in the recorded image stacks. By combining the two approaches, we intend to investigate whether the cellular response to external forces leads to a change in the observed buckling wavelength. It needs to be noted, however, that the images for sheared cells were recorded by epifluorescence microscopy (see section 3.3 , which has a lower resolution as compared to confocal microscopy used during our studies of buckling events. Since the resolution plays a key role in accessing the bundle diameter and thus the number of filaments in a bundle, we abstain from a full quantitative evaluation of buckling events under shear stress and restrict our investigation to a qualitative assertion of the buckling wavelength. 

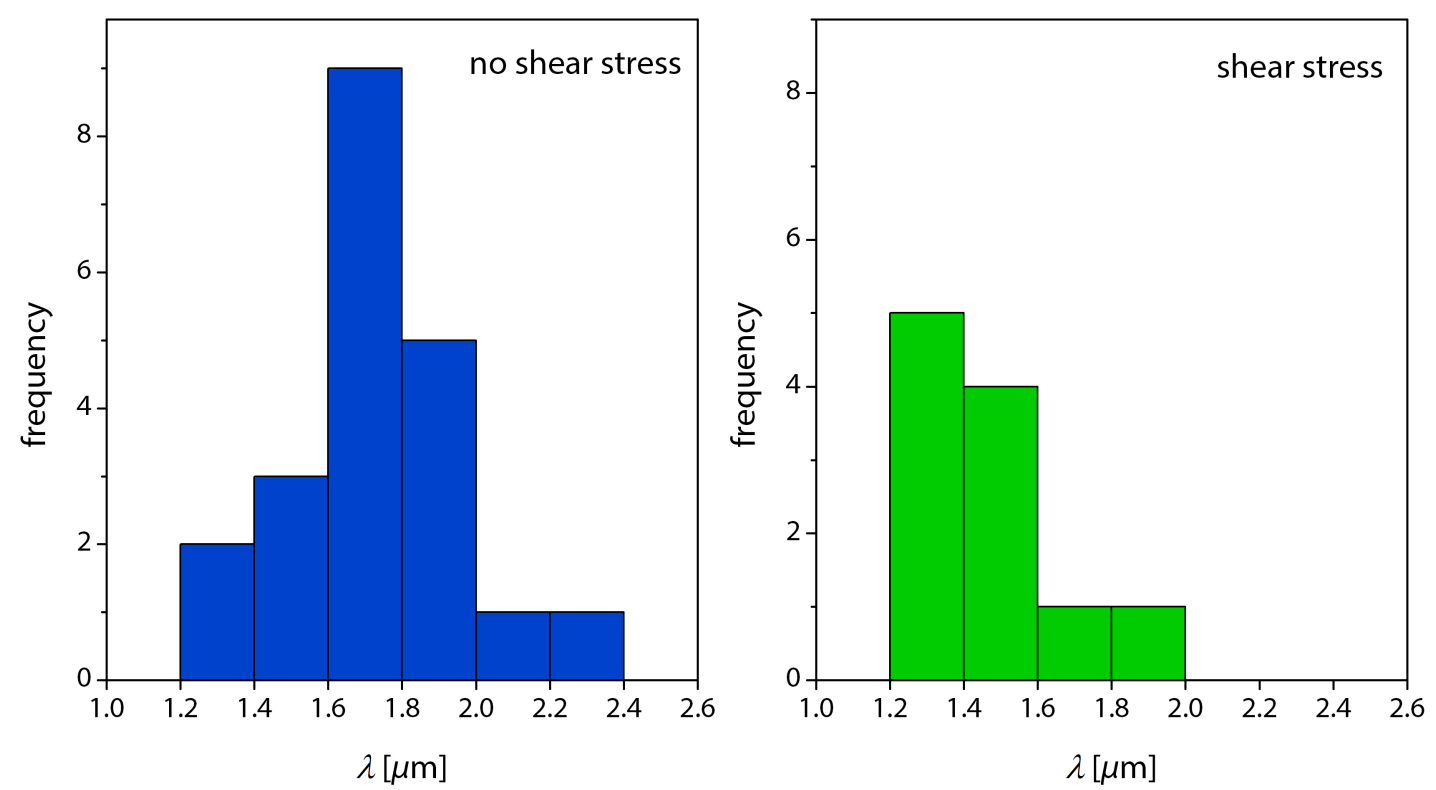

Figure 5.20: Comparison of buckling events in cells under shear and no-shear conditions. Without external forces, the observed buckling wavelengths are larger on average. In addition, the number of buckling events that are assessable is reduced when shear stress is applied to the cell.

In the following, we compare the buckling wavelengths occurring in cells before the start of micro-flow with those encountered within 5 to 20 min after the onset of flow. In total, we find a reduction of the buckling wavelength when shear stress is applied to the cells. Figure 5.20 shows an overview of the determined buckling wavelength in non-sheared (blue) and sheared cells (green).

As described earlier, the wavelength $\lambda$ is defined by the strength of coupling between the KIFs in a bundle, the elastic properties of a single keratin filament and the elastic modulus of the surrounding matrix, i.e. according to section 5.3 .2 .

$$
\lambda=2 \pi\left(\frac{N^{\gamma} \cdot L_{\mathrm{PF}} \cdot k_{\mathrm{B}} T}{\alpha}\right)^{1 / 4} \quad, \gamma \in[1,2] .
$$

Since the elastic properties of a single filament (i.e. $L_{\mathrm{PF}}$ ) are unlikely to change under the influence of shear stress, the reduced wavelength can be explained either by a weaker linkage between the KIFs inside a bundle or by an increase in the elastic modulus of the surrounding matrix. In our study, there have been no indications for a reduction of the coupling strength within a bundle as a consequence of the application of shear forces to cells. Studies from Sivaramakrishnan et al. or Flitney et al. rather support the idea of a continuously strong linkage between the filaments inside a bundle 38,72 under shear conditions. According to the two groups, thicker 
bundles form under the influence of shear stress and there is an enhanced incorporation of free keratin particles into the preexisting keratin network. We therefore conclude upon increased attractive interactions between keratin bundles and keratin filaments/particles, when shear stress is present. This circumstance is in direct opposition to a weakening of bundle inter-linkage, which is why we disregard the idea to explain our data.

By contrast, we do find evidence for a change of the elastic modulus of the cell. As described in chapter 4, we observe a reduction of keratin bundle motion when cells are exposed to shear forces. This behavior is explainable by an increased motor protein activity, which finally enhances the effective cell rigidity. This rise in the elastic modulus results in an increase of $\alpha$, which would account for a reduced buckling wavelength under shear conditions. Our investigation of buckling events in sheared cells thus supports our earlier findings from chapter 4.

Furthermore, our observations indicate that the number of buckling events per time interval is reduced under the influence of shear forces. For our dataset, where we have recorded the same amount of images for each condition with and without shear forces, we find 32 buckling events when the flow is absent and 16 events when shear stress is present. This change agrees with the expected behavior for an increase in the stiffness of the surrounding medium. Since the critical force which is needed to induce buckling is given by $f_{\text {crit }}=2 \sqrt{\kappa \alpha}$ (see section 5.1 .4 for details), the elastic modulus of the surroundings basically defines the critical force. As our observation of the keratin network in sheared cells suggest an enhanced stiffness of the cell, $f_{\text {crit }}$ increases in accordance, so that the number of buckling events observed reduces to those events which are caused by forces above the new and higher threshold $f_{\text {crit }}$.

\subsubsection{Possible Optimizations}

As described earlier, a determination of the persistence length of a freely fluctuating keratin filament would enhance the precision of our findings. It would allow us, in particular, to analyze the local shear modulus of the matrix surrounding the bundle: The constant in equation (5.55) describes an intercept, which is dependent on the persistence length of one filament $L_{\mathrm{PF}}$ and basically the stiffness of the cytoplasm $(\alpha(G))$. By fitting the linear regime of our data and extracting a value for the intercept, we could thus use the exact knowledge of one parameter to conclude upon the other.

Our results could be also improved by an optimization of the spline fit, which was used to access the buckling shape of the bundles. For this purpose, we suggest 


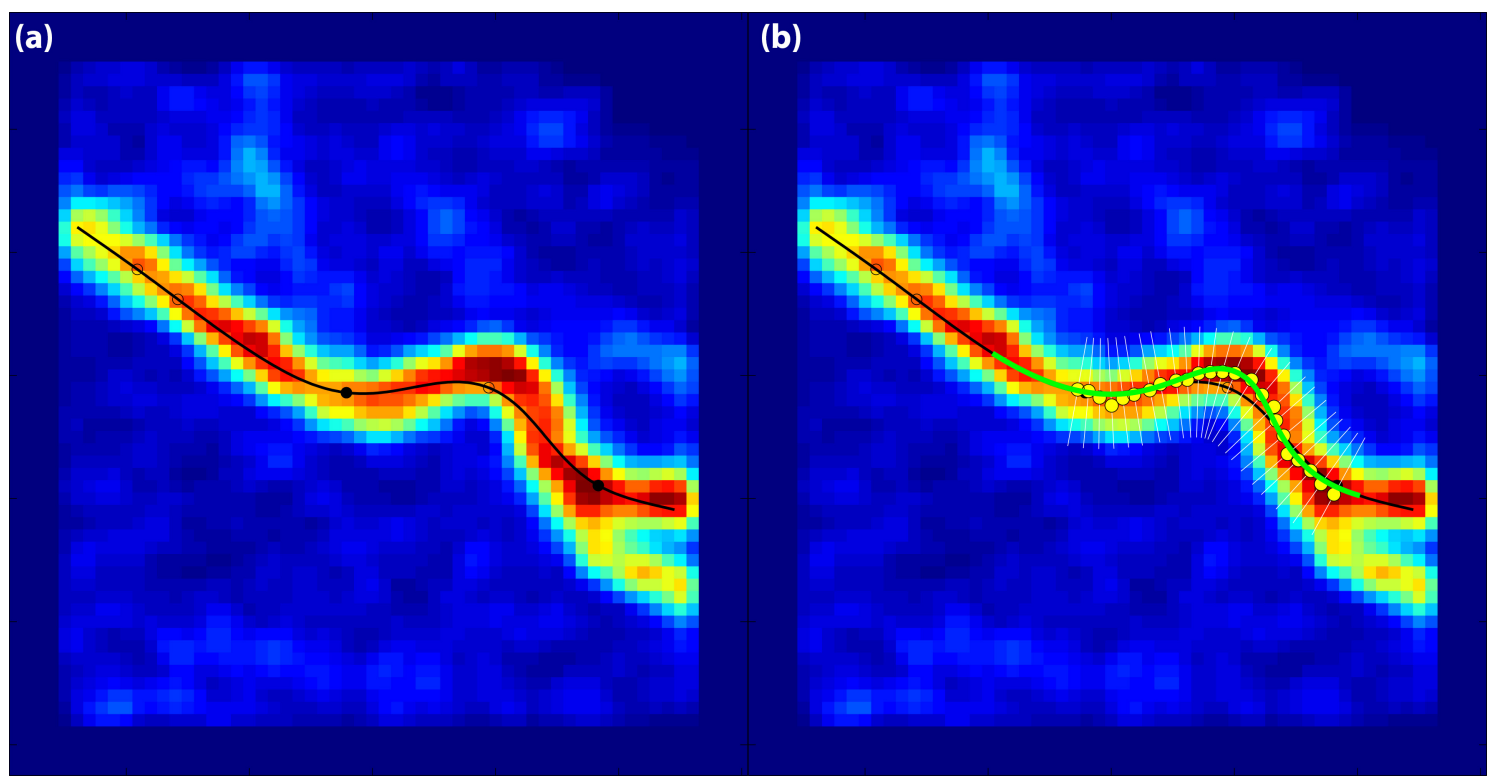

Figure 5.21: An optimization regarding the identification of the shape of the bundle could be achieved by a recursive algorithm which uses the information gained during the determination of the bundle diameter. To estimate the bundle diameter, Gaussian functions are fitted to profiles perpendicular to the bundle contour. The fit parameter specifying the center of the Gaussian functions (yellow dots) could therefore be reused to improve the spline fit of the bundle shape (possible result is sketched as a green line).

taking advantage of the information obtained from the determination of the bundle diameters: As we fit Gaussian functions to lines perpendicular to the identified spline curve, we could use the maximum of the Gaussian to redefine the center of the spline fit. A recursive algorithm could then lead to a spline fit which more precisely follows the form of the bundle. This improvement could also enhance the quality of the identification of points with maximal curvature. An example is sketched as a green curve in figure 5.21 b. However, the determination of the points where the curvature is maximal is sufficiently accurate for the current analysis of buckling wavelengths. The suggested optimization is therefore of greater interest for studies which investigate e.g. the growth rates of buckling events.

Finally, we could enhance image quality by using super-resolution microscopy techniques like STED. In principle, higher resolutions as compared to confocal microscopy are achievable with this technique. To this effect, donut-shaped regions around the focal spot of the normal excitation signal are illuminated with additional light, which depletes the signal from non-centralized fluorophores by stimulated emission. The usage of STED microscopy would allow for a better determination of the bundle diameter and may also improve the quality of the spline fit along the bundle. 


\section{Summary and Conclusion}

We have performed different experiments to shed light on the functionality of keratin networks and the mechanical properties of bundles of keratin intermediate filaments in living cells. One set of experiments aimed for the evaluation of short-termed responses of cells to shear stress with regard to their keratin network. To this end, the cells were placed in a microfluidic channel, where they were exposed to controlled shear stress by applying a micro-flow while they were observed in situ by fluorescence microscopy. We investigated the motion of the network resulting from active motor protein forces in comparison between no-shear and shear conditions.

For the analysis, we opted for different approaches to get access to network motion characteristics. First, we followed a global approach, where the calculation of image-to-image cross-correlations gives answers about the time dependent similarity of the entire network between two points in time. Thus, we were able to determine characteristic time-scales of the network motion. We found that the cross-correlation decays more slowly for sheared cells, meaning that the network is more persistent under shear stress over time. The result was confirmed by our analysis of network node trajectories. This local approach allows for in-detailed analyses of the motion of individual keratin network nodes. By calculating radii of gyration, we found that the keratin network nodes move less after application of shear stress to the cells. This finding is emphasized by the investigation of the mean-square-displacement of the nodes. Here, we calculated an effective diffusion constant to characterize the node displacement over time. We found that this constant decreases upon application of external forces to cells.

Since the keratin network is a sufficiently stiff structure, its motion cannot be explained by thermal forces. As IFs are in general apolar and are thus not suitable for directed motor protein movement along their filamentous structure, we identified a distinct force contribution of actin-associated myosin II by treating cells with 
blebbistatin, which ultimately inhibited the keratin bundle motion. Consequently, we concluded upon a change of the myosin II motor protein activity under shear stress. Indeed, studies have shown that in epithelial cells, which are exposed to shear stress, the concentration of $\mathrm{Ca}^{2+}$-ions is increased. $\mathrm{Ca}^{2+}$-ions are, in turn, known to play a crucial role in regulating the activity of myosin II motor proteins. We conclude that the increase of calcium ion concentrations, as a cellular response to shear forces, leads to an increased myosin II force generation. As a result, the effective rigidity of the actin network seems to increase, which is transmitted to the keratin scaffold by cross-linking proteins and becomes ultimately apparent as a reduced motion of the keratin network. We propose this response of the cell to be a protective mechanism that is triggered by external forces with the aim to help the cell withstand potentially harmful mechanical influences and to guarantee its integrity.

In a comparison with previous research, we have been able to contribute to the overall understanding of keratin-related cellular adaptation to external, mechanical influences: While former investigations have focused on the shear stress induced change of the keratin network morphology within hours and have linked these morphological rearrangements to alterations in the phosphorylation state of keratin, we have investigated the active cellular response to shear stress which occurs within minutes. From prior publications, it is known that on time scales of hours the application of external forces leads to a finally enhanced keratin network structure with thicker bundles and a more homogeneous mesh size distribution. Adding to this knowledge, we have identified in our experiments cellular processes which become effective on the time-scale of minutes and ultimately aim for the same goal of a finally fortified intracellular scaffold.

The other main set of experiments in this thesis aimed for the investigation of the mechanical properties of bundles consisting of keratin filaments. While previous studies of keratin networks have had to rely on experiments in vitro (e.g. rheology) or indirect measurements of the mechanical properties of the whole cell (e.g. AFM or deformation experiments), we were able to analyze the specific properties of keratin bundles in living cell directly. To this end, we have observed naturally occurring buckling events in vivo using confocal fluorescence microscopy. Based on these data, we have calculated the buckling wavelength and inferred the number of constituents of the bundle.

For our investigations, we have adapted the theory of constrained buckling, which was originally developed to describe buckling events of microtubules, and extended it to take the bundle characteristics into account, as individual filaments may slide 
against each other. To this end, we have introduced a coupling parameter, which indicates the strength of linkage between filaments. According to our model, the buckling wavelength and the number of filaments in a bundle are connected by a power law, where the coupling parameter is part of the exponent and the prefactor contains information about the shear modulus of the surrounding matrix in combination with the persistence length of an individual filament.

The evaluation of our data based on the established power law shows that the coupling between filaments within a bundle is primarily strong. Consequently, the movement of filaments against each other is highly limited and a bundle behaves mainly as one single rod. As a result, keratin bundles show high bending rigidities, which are in the same order of magnitude as for microtubules. Likewise, the buckling wavelengths of keratin and microtubules in cells are comparable. The data suggest that the keratin network plays a key role in determining the mechanical properties of the cell, like its stiffness. Interestingly, our data further indicate that filaments, which constitute bundles of larger diameters, have weaker inter-linkage. This feature can be understood when taking into account that in cells the bundle formation and thus also the thickness is regulated by proteins. As the diameter of keratin bundles is restricted by protein intervention, a weakening of the linkage of outer filaments seems plausible. Additionally, we have found evidence from TEM images that multiple bundles tend to attach to each other. The resulting bond between the two cylindrical bundles is relatively small for geometrical reasons and thus potentially weaker in the end.

Finally, we have investigated the influence of shear stress, which is applied to cells, on buckling events of keratin bundles. We have found that the buckling wavelength is reduced when shear forces are affecting the cells. This finding is in accordance with our previous results of a shear stress induced stiffening of the surrounding matrix - in particular the actin network - due to increased motor protein activity. The enhanced rigidity of the surrounding medium of the bundles, in turn, limits the ability of a buckled bundle to form large waveforms and increases the strength of the force which is required to cause a buckling event. Consequently, we have also observed a reduction in the number of buckling events per time in cells which are exposed to shear forces.

Altogether, our findings from both sets of experiments support the idea that keratin acts as a mechanical buffer system in the cell, partly in combination with actin, and that it has a distinct contribution to the mechanical properties of the cell. Future studies will hopefully give new insights into the interactions between actin and keratin networks, for which our experiments have given strong evidence. 


\section{Additional Datasets}

\section{Trajectories of Keratin Network Nodes}

In the following, the trajectories of nodes of two cells are shown as exemplary data sets. The nodes of the first cell lead to an average radius of gyration $R_{\mathrm{g}}$ of $0.165 \pm 0.02 \mu \mathrm{m}$ for no-shear conditions and $0.128 \pm 0.01 \mu \mathrm{m}$ when shear forces are present. For this data set the effective diffusion coefficient $D_{\text {eff }}$ lowers from $(5.78 \pm 2.0) \cdot 10^{-16}\left[\mathrm{~m}^{2} / \mathrm{s}\right]$ to $(2.57 \pm 0.6) \cdot 10^{-16}\left[\mathrm{~m}^{2} / \mathrm{s}\right]$ under shear stress (all error are SEM).

The second example shows a reduction of $R_{g}$ from originally $0.261 \pm 0.02 \mu \mathrm{m}$ to $0.133 \pm 0.01 \mu \mathrm{m}$ when external shear is applied. Here, $D_{\text {eff }}$ is also reduced from $(4.27 \pm 1.2) \cdot 10^{-16}\left[\mathrm{~m}^{2} / \mathrm{s}\right]$ to $(1.71 \pm 0.5) \cdot 10^{-16}\left[\mathrm{~m}^{2} / \mathrm{s}\right]$. 


\section{Example 1}

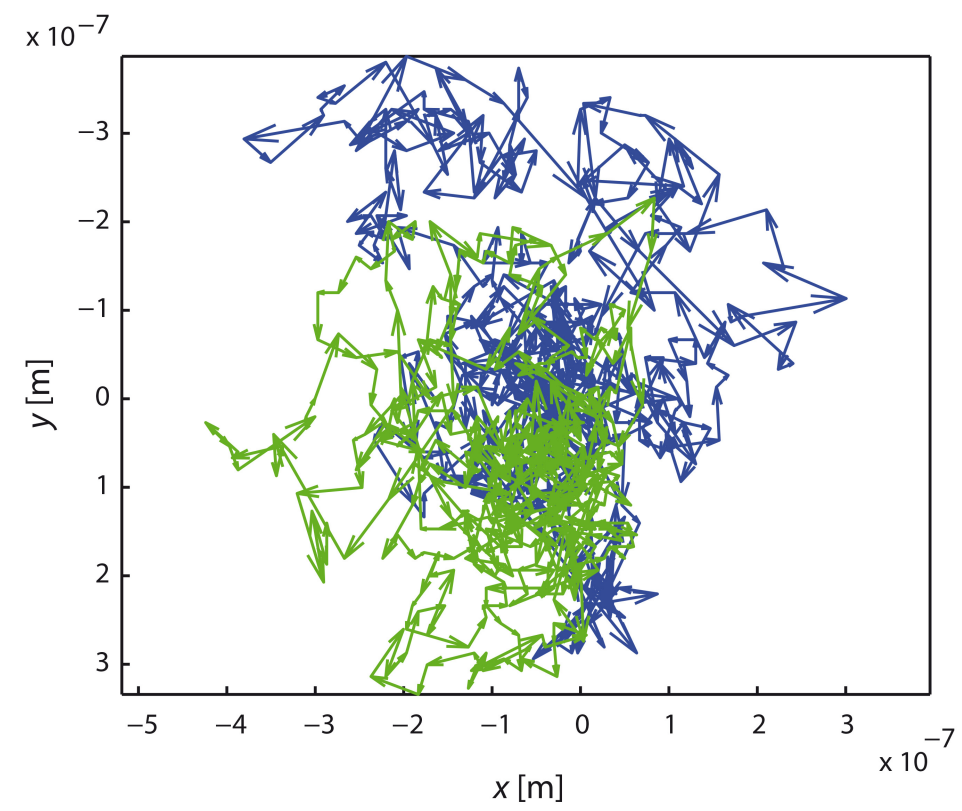

$R_{\mathrm{g}, \text { no-shear }}=1.841 \cdot 10^{-7} \mathrm{~m}, R_{\mathrm{g}, \text { shear }}=1.456 \cdot 10^{-7} \mathrm{~m}$;

$D_{\text {eff,no-shear }}=5.60 \cdot 10^{-16} \mathrm{~m}^{2} / \mathrm{s}, D_{\text {eff,shear }}=2.50 \cdot 10^{-16} \mathrm{~m}^{2} / \mathrm{s}$;

data: 2012_06_01_exp_001 node-id 19511

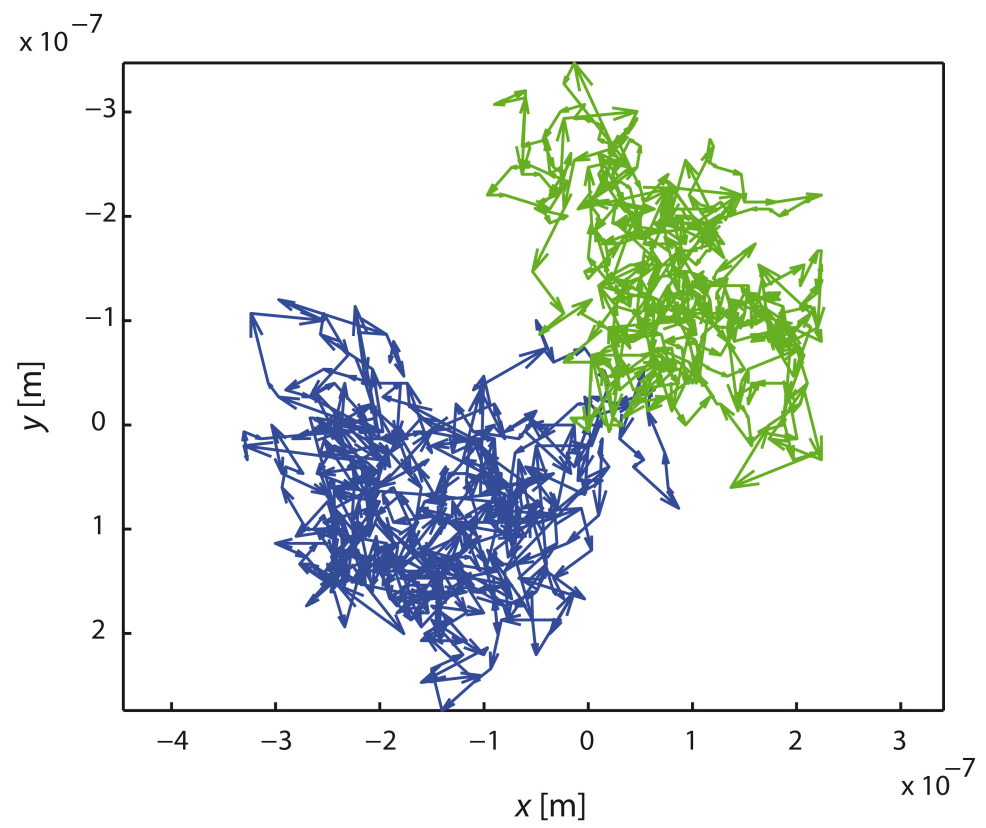

$R_{\mathrm{g}, \text { no-shear }}=1.139 \cdot 10^{-7} \mathrm{~m}, R_{\mathrm{g}, \text { shear }}=1.046 \cdot 10^{-7} \mathrm{~m}$;

$D_{\text {eff,no-shear }}=3.30 \cdot 10^{-16} \mathrm{~m}^{2} / \mathrm{s}, D_{\text {eff,shear }}=1.94 \cdot 10^{-16} \mathrm{~m}^{2} / \mathrm{s}$;

data: 2012_06_01_exp_001 node-id 67362 


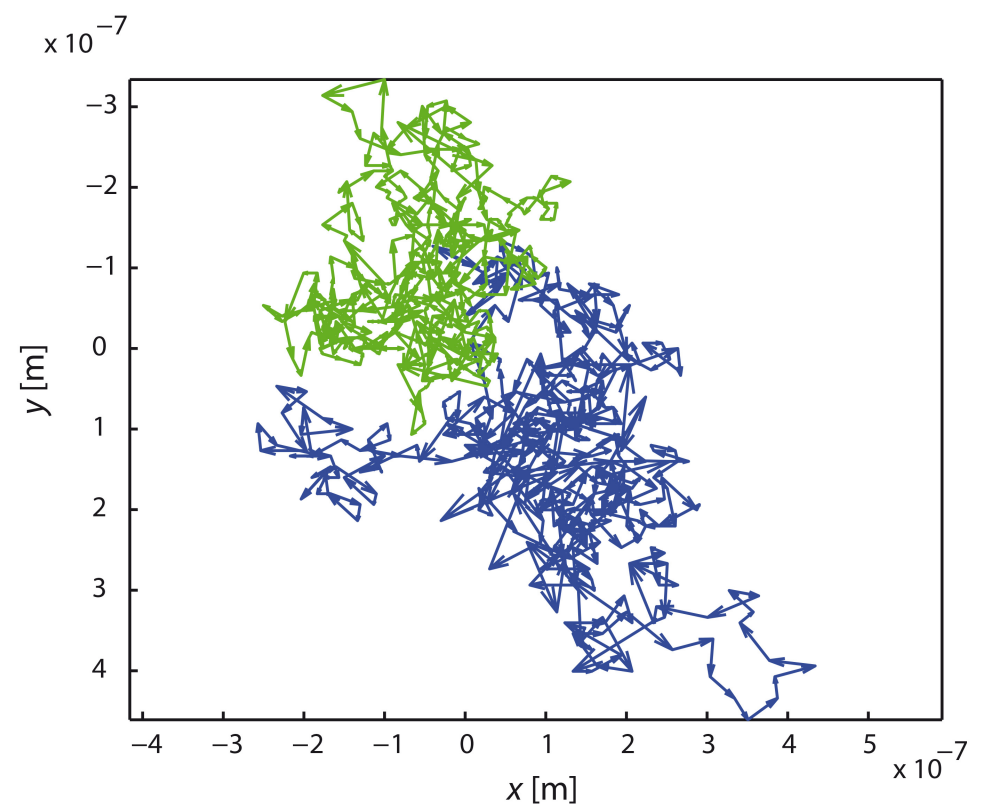

$R_{\mathrm{g}, \text { no-shear }}=1.627 \cdot 10^{-7} \mathrm{~m}, R_{\mathrm{g}, \text { shear }}=1.128 \cdot 10^{-7} \mathrm{~m}$;

$D_{\text {eff,no-shear }}=2.81 \cdot 10^{-16} \mathrm{~m}^{2} / \mathrm{s}, D_{\text {eff,shear }}=1.58 \cdot 10^{-16} \mathrm{~m}^{2} / \mathrm{s}$;

data: 2012_06_01_exp_001 node-id 85064

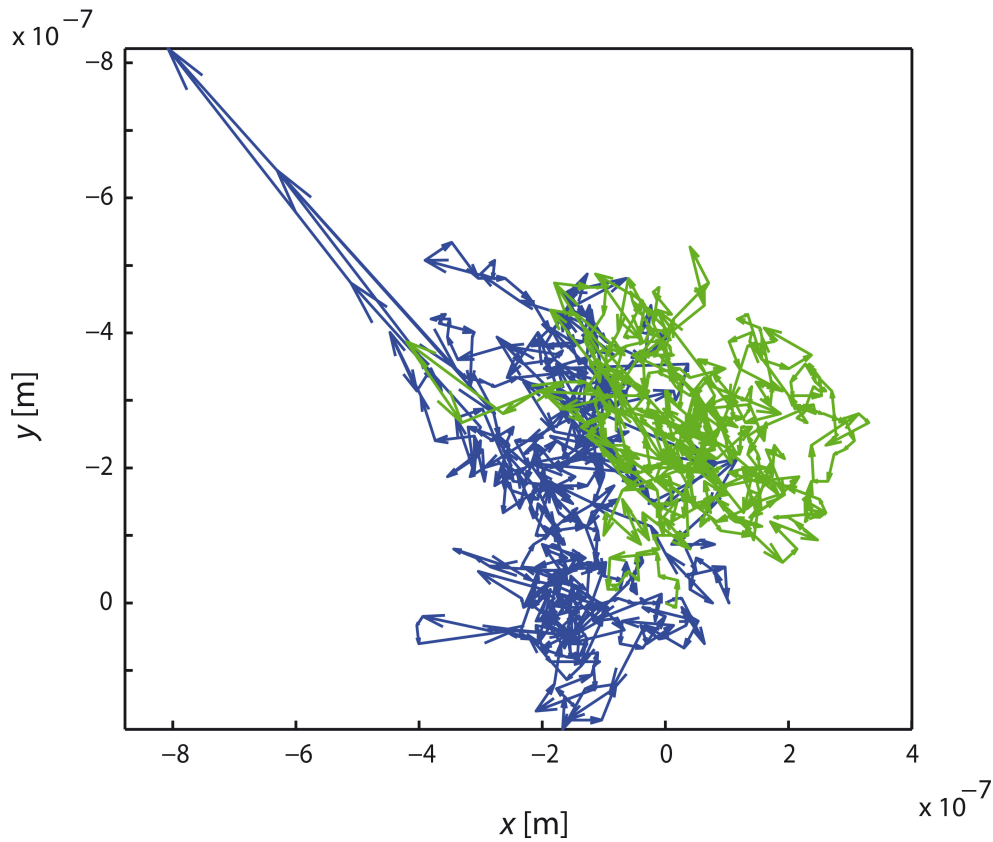

$R_{\mathrm{g}, \text { no-shear }}=1.997 \cdot 10^{-7} \mathrm{~m}, R_{\mathrm{g}, \text { shear }}=1.489 \cdot 10^{-7} \mathrm{~m}$;

$D_{\text {eff,no-shear }}=1.14 \cdot 10^{-15} \mathrm{~m}^{2} / \mathrm{s}, D_{\text {eff,shear }}=4.25 \cdot 10^{-16} \mathrm{~m}^{2} / \mathrm{s}$;

data: 2012_06_01_exp_001 node-id 94829 


\section{Example 2}

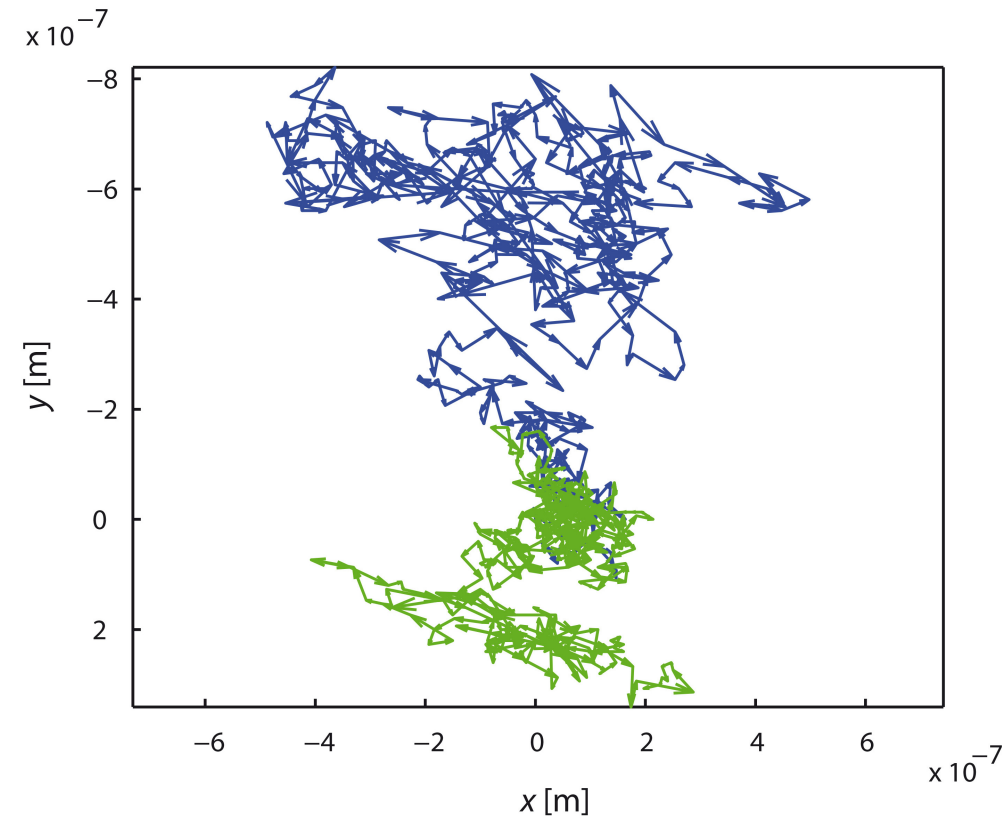

$R_{\mathrm{g}, \text { no-shear }}=2.966 \cdot 10^{-7} \mathrm{~m}, R_{\mathrm{g}, \text { shear }}=1.446 \cdot 10^{-7} \mathrm{~m}$;

$D_{\text {eff,no-shear }}=6.58 \cdot 10^{-16} \mathrm{~m}^{2} / \mathrm{s}, D_{\text {eff,shear }}=2.62 \cdot 10^{-16} \mathrm{~m}^{2} / \mathrm{s}$;

data: 2012_06_01__exp_002 node-id 49542

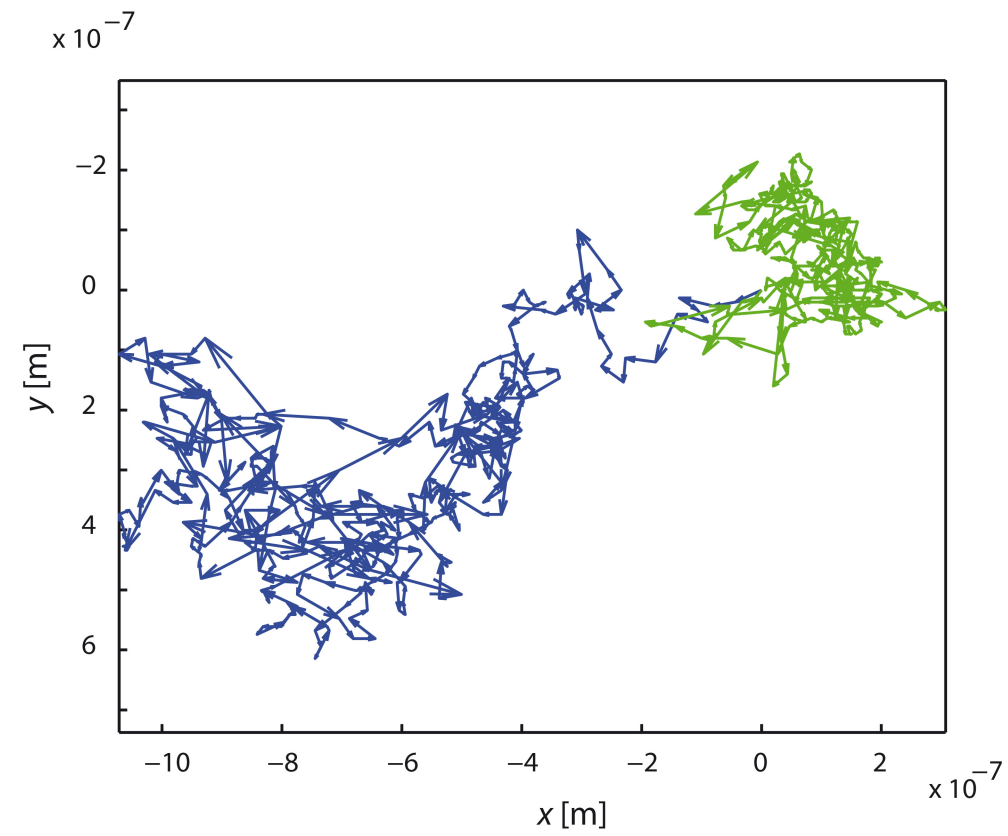

$R_{\mathrm{g}, \text { no-shear }}=2.660 \cdot 10^{-7} \mathrm{~m}, R_{\mathrm{g}, \text { shear }}=1.043 \cdot 10^{-7} \mathrm{~m}$;

$D_{\text {eff,no-shear }}=3.61 \cdot 10^{-16} \mathrm{~m}^{2} / \mathrm{s}, D_{\text {eff,shear }}=1.31 \cdot 10^{-16} \mathrm{~m}^{2} / \mathrm{s}$;

data: 2012_06_01_exp_002 node-id 52279 


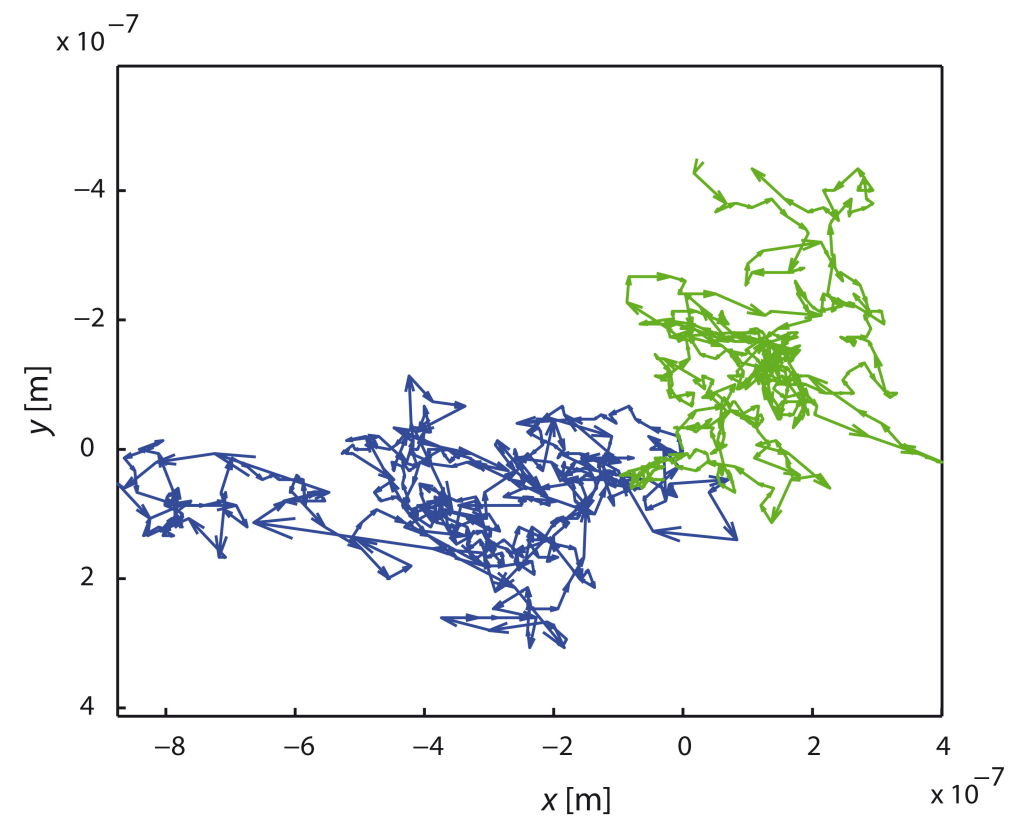

$R_{\mathrm{g}, \text { no-shear }}=2.210 \cdot 10^{-7} \mathrm{~m}, R_{\mathrm{g}, \text { shear }}=1.515 \cdot 10^{-7} \mathrm{~m}$;

$D_{\text {eff,no-shear }}=2.62 \cdot 10^{-16} \mathrm{~m}^{2} / \mathrm{s}, D_{\text {eff,shear }}=1.20 \cdot 10^{-16} \mathrm{~m}^{2} / \mathrm{s}$;

data: 2012_06_01_exp_002 node-id 98419 


\section{TEM Preparation}

The following preparation for transmission electron microscopy was performed and described in the subsequent form by Dr. Wiebke Möbius (personal communication).

SK8/18-2 cells were fixed by adding $2 \mathrm{x}$ concentrated fixative ( $8 \%$ formaldehyde, $5 \%$ glutaraldehyde in $0.1 \mathrm{M}$ phosphate buffer $\mathrm{pH} 7.3$ ) to the culture medium. After removal of the supernatant after 5 min the fixation was continued with $4 \%$ formaldehyde and $2.5 \%$ glutaraldehyde in $0.1 \mathrm{M}$ phosphate buffer $\mathrm{pH} 7.3$ for at least $4 \mathrm{~h}$. Then the cells were postfixed in $1 \%$ OsO4 (ChemPur) in $0.1 \mathrm{M}$ phosphate buffer and embedded in Epon (Serva) after dehydration with ethanol and en bloc staining with $1.5 \%$ uranyl acetate (Merck) and 1.5\% tungstophosphoric acid (Merck) in $70 \%$ ethanol. Ultrathin sections of cultured cells were cut parallel to the substrate using an Ultracut S Ultramicrotome (Leica, Vienna, Austria) and stained with an aqueous solution of $4 \%$ uranyl acetate followed by lead citrate [138. Sections were analyzed with a LEO EM912 Omega (Zeiss, Oberkochen) and digital micrographs were obtained with an on-axis $2048 \times 2048$-CCD camera (TRS, Moorenweis). 


\section{List of Figures}

$2.1 \quad$ Image of parts of the cytoskeleton: keratin and actin . . . . . . . . 4

2.2 Structure of an IF protein $\ldots \ldots \ldots$. . . . . . . . . . . . . 5

2.3 SEM images of a keratin network . . . . . . . . . . . . . . . . . . . . . . . . . . . . . . . . . .

2.4 Keratin cycle in cells . . . . . . . . . . . . . . . . . . . 11

$2.5 \quad$ Buckling in cells . . . . . . . . . . . . . . . . . . . . . . . . 14

$3.1 \quad$ Sketches of an epifluorescence and a confocal microscopy setup . . . . 19

3.2 Jablonski diagram for fluorescence . . . . . . . . . . . . . . . . . . . . . . . . . . . . . . .

3.3 Spectra of U-MNIBA3 filter-cube . . . . . . . . . . . . . . . . . 21

3.4 Fabrication of microfluidic devices from PDMS using photolithography 24

4.1 Definition of shear stress regarding Newtonian fluids . . . . . . . . . . 28

$4.2 \quad 3 \mathrm{D}$ simulation of the velocity and shear stress inside a microfluidic channel . . . . . . . . . . . . . . . . . . . 31

$4.3 \quad 2 \mathrm{D}$ excerpt of the velocity and shear stress distribution depending on the channel height . . . . . . . . . . . . . . . . . . . . 32

4.4 Shear stress distribution at the channel's bottom. . . . . . . . . . . . 33

4.5 Inverted epifluorescence microscopy image of SK8/18-2 cells . . . . . 34

4.6 Comparison between a raw image and a processed image of a SK8/182 cell . . . . . . . . . . . . . . . . . 35 
4.7 Comparison of an automatic node tracking procedure with a manual approach . . . . . . . . . . . . . . . . . . . 38

4.8 Example of image-to-image cross-correlations (sheared vs non-sheared

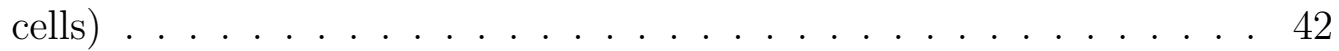

4.9 Overview over all image-to-image cross-correlations (sheared vs nonsheared cells) . . . . . . . . . . . . . . . . . . . . . . 43

4.10 Average and histogram of decay times for cross-correlations (sheared vs non-sheared cells) . . . . . . . . . . . . . . . . . . . . . . . 44 44

4.11 Example of a trajectory of a KIF network node (sheared vs nonsheared cells) . . . . . . . . . . . . . . . . . 4 45

4.12 Average and histogram of the radii of gyration (sheared vs nonsheared cells) . . . . . . . . . . . . . . . . 4 46

4.13 Example mean-square-displacement of a KIF network node (sheared vs non-sheared cells $] \ldots \ldots$. . . . . . . . . . . . . . . . . . 4 47

4.14 Average and histogram of the effective diffusion constants (sheared vs non-sheared cells) . . . . . . . . . . . . . . . . . . 48

4.15 Treatment of SK8/18-2 cells with blebbistatin . . . . . . . . . . . . . 49

4.16 Overview of the response of intracellular keratin to shear stress on different time scales . . . . . . . . . . . . . . . . . . 53

4.17 Actin and keratin networks in SK8/18-2 cells . . . . . . . . . . . . 56

5.1 Sketch of an Euler and a constrained buckling event . . . . . . . . . . 60

5.2 Bending process of a bar . . . . . . . . . . . . . . . . . 61

5.3 Sketch of a bent line . . . . . . . . . . . . . . . . . . . . 62

5.4 Sketch of Euler buckling . . . . . . . . . . . . . . . . . . 63

5.5 Sketch of a filament where the left end is fixed . . . . . . . . . . . . . 65

5.6 The buckling behavior of a bundle is dependent on the coupling between bundle constituents . . . . . . . . . . . . . . . . . 71

5.7 Identification of buckling events . . . . . . . . . . . . . . 74

5.8 Steps during buckling event evaluation . . . . . . . . . . . . . . . 75

$5.9 \quad$ Visualization of spline fitting notations $\ldots \ldots \ldots$. . . . . . . 77

5.10 Visualization of the curvature of the spline fit . . . . . . . . . . . 78

5.11 Evaluation of the buckling wavelength and the bundle diameter . . . 79

5.12 Determination of the FWHM of Gaussians fitted to images of beads . 81

5.13 TEM images of keratin bundles in SK8/18-2 cells . . . . . . . . . . . 83

5.14 Numeric approach to determine the number of filaments inside a bundle with a certain diameter . . . . . . . . . . . . . . . . . . . . 84 
5.15 Histograms of buckling events showing the distribution of bundle diameters and observed buckling wavelengths . . . . . . . . . . . . 8 85

5.16 Buckling wavelength $\lambda$ plotted against the number $N$ of filaments in a bundle . . . . . . . . . . . . . . . . . . . . 86

5.17 Buckling wavelength plot with additional error estimates for the elastic modulus of the surrounding matrix . . . . . . . . . . . . . . 88

5.18 Persistence length $L_{\mathrm{PB}}$ of a bundle as a function of the coupling parameter $\gamma \ldots \ldots \ldots$. . . . . . . . . . . . . . . . . 90

5.19 TEM image of a keratin K8K18 filament bundle composed of two sub-bundles . . . . . . . . . . . . . . . . . . . . . . . . . . 92

5.20 Comparison of buckling events in cells under shear and no-shear conditions . . . . . . . . . . . . . . . . . . 95

5.21 Possible optimization for the identification of the bundle shape . . . . 97 


\title{
Abbreviations and Symbols
}

\section{Abbreviations}

\author{
AFM ....... atomic force microscopy \\ ATP ....... adenosine triphosphate \\ CC ......... cross-correlation \\ DMEM ..... Dulbecco's modified Eagle medium \\ EDTA ...... ethylenediaminetetraacetic acid \\ FCS ....... fetal calf serum \\ FEM ...... finite element method \\ FRAP ...... fluorescence recovery after photobleaching \\ FWHM .... full width at half maximum \\ IF ........ intermediate filament \\ KIF ....... keratin intermediate filament \\ MF ........ microfilament \\ MLCK ..... myosin light-chain kinase \\ MSD ...... mean-square-displacement \\ MT ........ microtubule \\ PBS ....... phosphate buffered saline \\ PSF ....... point spread function \\ PDMS ..... polydimethylsiloxane \\ Pen-Strep ... penicillin-streptomycin \\ PKC ....... protein kinase $\mathrm{C}$ \\ SEM ........ scanning electron microscopy \\ STED ...... stimulated emission depletion
}


TEM ...... transmission electron microscopy

ULF ....... unit-length-filament

\section{Most Important Symbols - Sheared Cells}

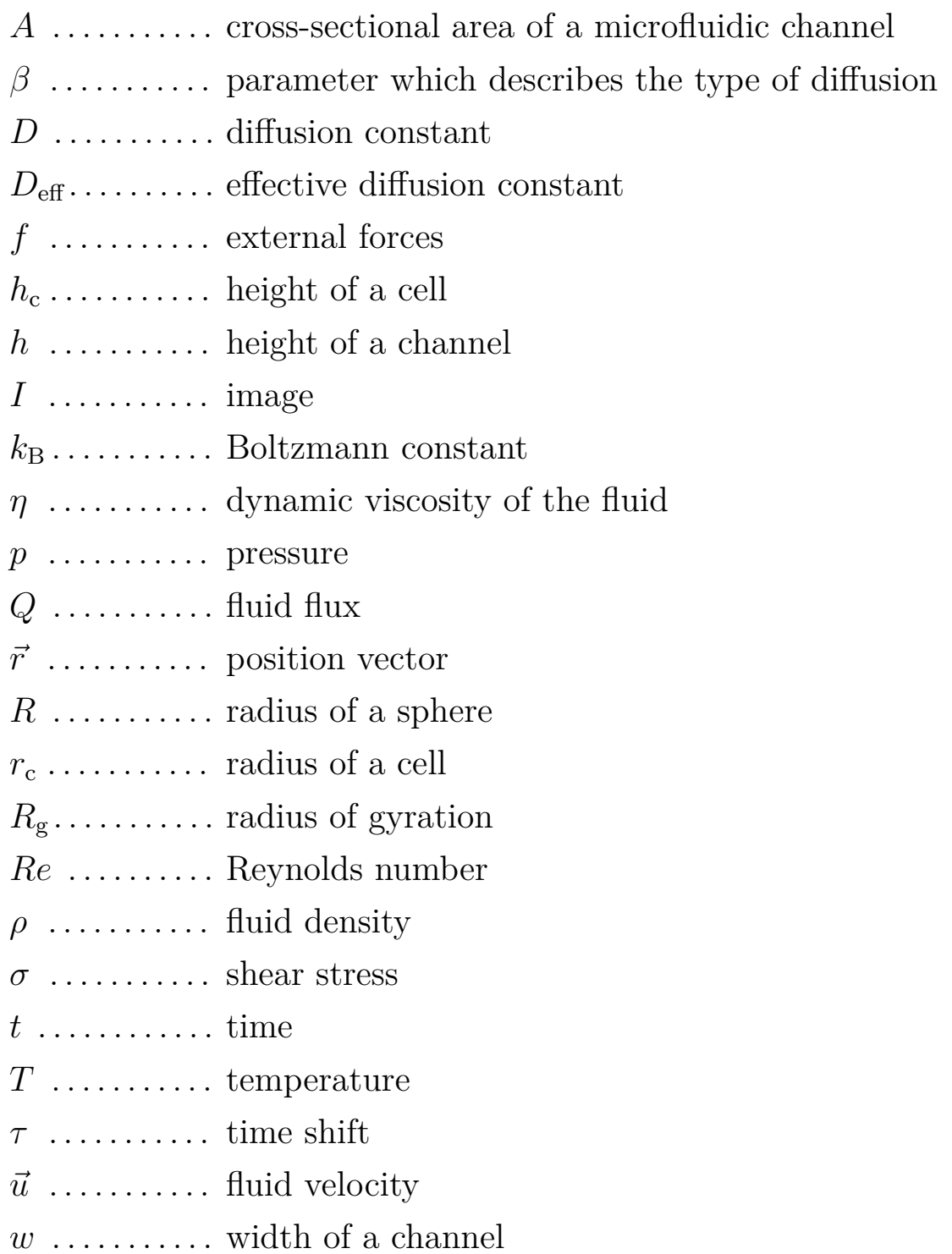

\section{Most Important Symbols - Buckling}

A.......... cross-sectional area of a bar

$\alpha \ldots \ldots \ldots$ parameter of the shear modulus 


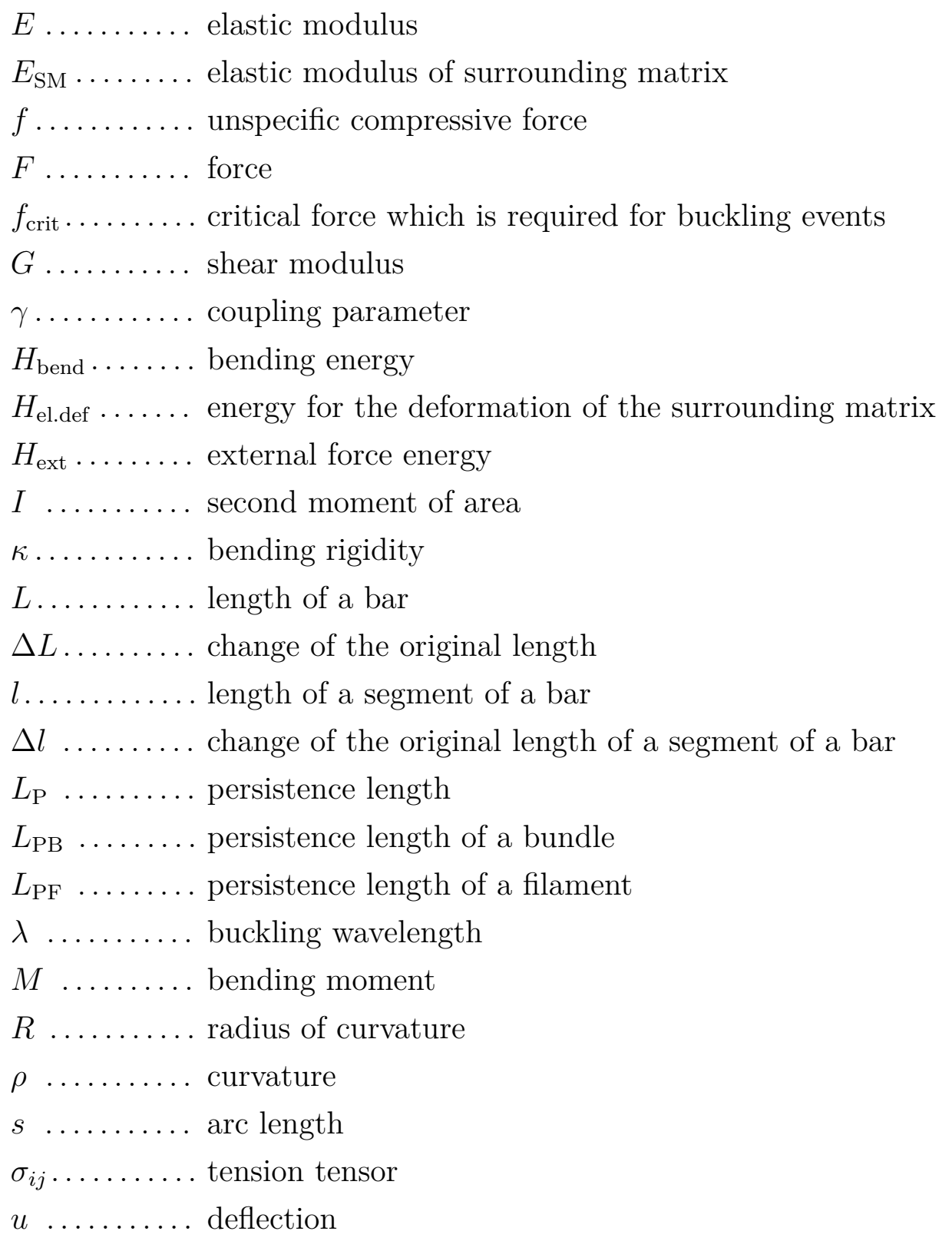




\section{Bibliography}

[1] B. Alberts, A. Johnson, J. Lewis, M. Raff, K . Roberts, and P Walter. Molecular Biology of the Cell. Garland Science, 5th edition, 2008.

[2] D.A. Fletcher and R.D. Mullins. Cell mechanics and the cytoskeleton. Nature, 463(7280):485-492, 2010.

[3] J.S. Kim, C.H. Lee, and P.A. Coulombe. Modeling the self-organization property of keratin intermediate filaments. Biophysical Journal, 99(9):2748-2756, 2010.

[4] L. Chang and R.D. Goldman. Intermediate filaments mediate cytoskeletal crosstalk. Nature Reviews Molecular Cell Biology, 5(8):601-613, 2004.

[5] J.E. Eriksson, T. Dechat, B. Grin, B. Helfand, M. Mendez, H.M. Pallari, and R.D. Goldman. Introducing intermediate filaments: from discovery to disease. The Journal of Clinical Investigation, 119(7):1763, 2009.

[6] H. Herrmann, S.V. Strelkov, P. Burkhard, and U. Aebi. Intermediate filaments: Primary determinants of cell architecture and plasticity. The Journal of Clinical Investigation, 119(7):1772, 2009.

[7] H. Herrmann and U. Aebi. Intermediate filaments: Molecular structure, assembly mechanism, and integration into functionally distinct intracellular scaffolds. Annual Review of Biochemistry, 73(1):749-789, 2004.

[8] J. Schweizer, P.E. Bowden, P.A. Coulombe, L. Langbein, E.B. Lane, T.M. Magin, L. Maltais, M.B. Omary, D.A.D. Parry, M.A. Rogers, and M.W. Wright. New consensus nomenclature for mammalian keratins. The Journal of Cell Biology, 174(2):169-174, 2006. 
[9] J. McKittrick, P.Y. Chen, S.G. Bodde, W. Yang, E.E. Novitskaya, and M.A. Meyers. The structure, functions, and mechanical properties of keratin. JOM, 64(4):449-468, 2012.

[10] S. Yamada, D. Wirtz, and P.A. Coulombe. Pairwise assembly determines the intrinsic potential for self-organization and mechanical properties of keratin filaments. Molecular Biology of the Cell, 13(1):382-391, 2002.

[11] R.L. Haines and E.B. Lane. Keratins and disease at a glance. Journal of Cell Science, 125(17):3923-3928, 2012.

[12] H.H. Bragulla and D.G. Homberger. Structure and functions of keratin proteins in simple, stratified, keratinized and cornified epithelia. Journal of Anatomy, 214(4):516-559, 2009.

[13] H. Herrmann, T. Wedig, R.M. Porter, E.B. Lane, and U. Aebi. Characterization of early assembly intermediates of recombinant human keratins. Journal of Structural Biology, 137(1):82-96, 2002.

[14] T. Lichtenstern, N. Mücke, U. Aebi, M. Mauermann, and H. Herrmann. Complex formation and kinetics of filament assembly exhibited by the simple epithelial keratins K8 and K18. Journal of Structural Biology, 177(1):54-62, 2012 .

[15] Christian Dammann. Personal communication. 022014.

[16] P. Janmey, D. Slochower, Y.H. Wang, Q. Wen, and A. Cebers. Polyelectrolyte properties of filamentous biopolymers and their consequences in biological fluids. Soft Matter, 2014.

[17] J.X. Tang and P.A. Janmey. The polyelectrolyte nature of F-actin and the mechanism of actin bundle formation. Journal of Biological Chemistry, 271(15):8556-8563, 1996.

[18] C. Dammann, B. Nöding, and S. Köster. Vimentin networks at tunable ionconcentration in microfluidic drops. Biomicrofluidics, 6(2):022009, 2012.

[19] L. Ma, S. Yamada, D. Wirtz, and P.A. Coulombe. A 'hot-spot' mutation alters the mechanical properties of keratin filament networks. Nature Cell Biology, 3(5):503-506, 2001.

[20] C.H. Lee and P.A. Coulombe. Self-organization of keratin intermediate filaments into cross-linked networks. The Journal of Cell Biology, 186(3):409-421, 2009. 
[21] J. Kayser, H. Grabmayr, M. Harasim, H. Herrmann, and A.R. Bausch. Assembly kinetics determine the structure of keratin networks. Soft Matter, 8(34):8873-8879, 2012.

[22] A. Leitner, T. Paust, O. Marti, P. Walther, H. Herrmann, and M. Beil. Properties of intermediate filament networks assembled from keratin 8 and 18 in the presence of $\mathrm{Mg}^{2+}$. Biophysical Journal, 103(2):195-201, 2012.

[23] R. Beck, J. Deek, J.B. Jones, and C.R. Safinya. Gel-expanded to gel-condensed transition in neurofilament networks revealed by direct force measurements. Nature Materials, 9(1):40-46, 2010.

[24] J.C. Troncoso, J.L. March, M. Häner, and U. Aebi. Effect of aluminum and other multivalent cations on neurofilaments in vitro: An electron microscopic study. Journal of Structural Biology, 103(1):2-12, 1990.

[25] P.A. Coulombe, O. Bousquet, L. Ma, S. Yamada, and D. Wirtz. The 'ins' and 'outs' of intermediate filament organization. Trends in Cell Biology, 10(10):420-428, 2000.

[26] P.M. Steinert, J.S. Cantieri, D.C. Teller, J.D. Lonsdale-Eccles, and B.A. Dale. Characterization of a class of cationic proteins that specifically interact with intermediate filaments. Proceedings of the National Academy of Sciences, 78(7):4097-4101, 1981.

[27] P. Listwan and J.A. Rothnagel. Keratin bundling proteins. Methods in Cell Biology, 78:817-827, 2004.

[28] S.C. Lee, I.G. Kim, L.N. Marekov, E.J. O'keefe, D.A. Parry, and P.M. Steinert. The structure of human trichohyalin. Potential multiple roles as a functional EF-hand-like calcium-binding protein, a cornified cell envelope precursor, and an intermediate filament-associated (cross-linking) protein. Journal of Biological Chemistry, 268(16):12164-12176, 1993.

[29] P. Krieg, M. Schuppler, R. Koesters, A. Mincheva, P. Lichter, and F. Marks. Repetin (rptn), a new member of the "fused gene" subgroup within the S100 gene family encoding a murine epidermal differentiation protein. Genomics, 43(3):339-348, 1997.

[30] T. Makino, M. Takaishi, M. Morohashi, and N. Huh. Hornerin, a novel profilaggrin-like protein and differentiation-specific marker isolated from mouse skin. Journal of Biological Chemistry, 276(50):47445-47452, 2001. 
[31] J.J. Jefferson, C.L. Leung, and R.K.H. Liem. Plakins: Goliaths that link cell junctions and the cytoskeleton. Nature Reviews Molecular Cell Biology, 5(7):542-553, 2004.

[32] G. Wiche. Role of plectin in cytoskeleton organization and dynamics. Journal of Cell Science, 111(17):2477-2486, 1998.

[33] S. Osmanagic-Myers, M. Gregor, G. Walko, G. Burgstaller, S. Reipert, and G. Wiche. Plectin-controlled keratin cytoarchitecture affects MAP kinases involved in cellular stress response and migration. The Journal of Cell Biology, 174(4):557-568, 2006.

[34] A. Sonnenberg and R.K.H. Liem. Plakins in development and disease. Experimental Cell Research, 313(10):2189-2203, 2007.

[35] D. Spazierer, J. Raberger, K. Groß, P. Fuchs, and G. Wiche. Stress-induced recruitment of epiplakin to keratin networks increases their resistance to hyperphosphorylation-induced disruption. Journal of Cell Science, 121(6):825833, 2008.

[36] M.B. Omary, N.O. Ku, G.Z. Tao, D.M. Toivola, and J. Liao. 'heads and tails' of intermediate filament phosphorylation: Multiple sites and functional insights. Trends in Biochemical Sciences, 31(7):383-394, 2006.

[37] P. Strnad, R. Windoffer, and R.E. Leube. In vivo detection of cytokeratin filament network breakdown in cells treated with the phosphatase inhibitor okadaic acid. Cell and Tissue Research, 306(2):277-293, 2001.

[38] E.W. Flitney, E.R. Kuczmarski, S.A. Adam, and R.D. Goldman. Insights into the mechanical properties of epithelial cells: The effects of shear stress on the assembly and remodeling of keratin intermediate filaments. The FASEB Journal, 23(7):2110-2119, 2009.

[39] P.A. Janmey, U. Euteneuer, P. Traub, and M. Schliwa. Viscoelastic properties of vimentin compared with other filamentous biopolymer networks. The Journal of Cell Biology, 113(1):155-160, 1991.

[40] Y.C. Lin, N.Y. Yao, C.P. Broedersz, H. Herrmann, F.C. MacKintosh, and D.A. Weitz. Origins of elasticity in intermediate filament networks. Physical Review Letters, 104(5):058101, 2010.

[41] C. Storm, J.J. Pastore, F.C. MacKintosh, T.C. Lubensky, and P.A. Janmey. Nonlinear elasticity in biological gels. Nature, 435(7039):191-194, 2005. 
[42] L. Ma, J. Xu, P.A. Coulombe, and D. Wirtz. Keratin filament suspensions show unique micromechanical properties. Journal of Biological Chemistry, 274(27):19145-19151, 1999.

[43] L. Kreplak, H. Bär, J.F. Leterrier, H. Herrmann, and U. Aebi. Exploring the mechanical behavior of single intermediate filaments. Journal of Molecular Biology, 354(3):569-577, 2005.

[44] D. Fudge, D. Russell, D. Beriault, W. Moore, E.B. Lane, and A.W. Vogl. The intermediate filament network in cultured human keratinocytes is remarkably extensible and resilient. PLoS One, 3(6):e2327, 2008.

[45] E. Fuchs and K. Weber. Intermediate filaments: Structure, dynamics, function and disease. Annual Review of Biochemistry, 63(1):345-382, 1994.

[46] T.M. Magin, P. Vijayaraj, and R.E. Leube. Structural and regulatory functions of keratins. Experimental Cell Research, 313(10):2021-2032, 2007.

[47] R. Windoffer, S. Wöll, P. Strnad, and R.E. Leube. Identification of novel principles of keratin filament network turnover in living cells. Molecular Biology of the Cell, 15(5):2436-2448, 2004.

[48] L. Ramms, G. Fabris, R. Windoffer, N. Schwarz, R. Springer, C. Zhou, J. Lazar, S. Stiefel, N. Hersch, U. Schnakenberg, et al. Keratins as the main component for the mechanical integrity of keratinocytes. Proceedings of the National Academy of Sciences, 110(46):18513-18518, 2013.

[49] K. Seltmann, A.W. Fritsch, J.A. Käs, and T.M. Magin. Keratins significantly contribute to cell stiffness and impact invasive behavior. Proceedings of the National Academy of Sciences, 110(46):18507-18512, 2013.

[50] R. Windoffer and R.E. Leube. Detection of cytokeratin dynamics by time-lapse fluorescence microscopy in living cells. Journal of Cell Science, 112(24):45214534, 1999.

[51] K.H. Yoon, M. Yoon, R.D. Moir, S. Khuon, F.W. Flitney, and R.D. Goldman. Insights into the dynamic properties of keratin intermediate filaments in living epithelial cells. The Journal of Cell Biology, 153(3):503-516, 2001.

[52] A. Kölsch, R. Windoffer, T. Würflinger, T. Aach, and R.E. Leube. The keratin-filament cycle of assembly and disassembly. Journal of Cell Science, 123(13):2266-2272, 2010. 
[53] S. Wöll, R. Windoffer, and R.E. Leube. Dissection of keratin dynamics: Different contributions of the actin and microtubule systems. European Journal of Cell Biology, 84(2):311-328, 2005.

[54] R. Windoffer, M. Beil, T.M. Magin, and R.E. Leube. Cytoskeleton in motion: The dynamics of keratin intermediate filaments in epithelia. The Journal of Cell Biology, 194(5):669-678, 2011.

[55] Y.H. Chou, F.W. Flitney, L. Chang, M. Mendez, B. Grin, and R.D. Goldman. The motility and dynamic properties of intermediate filaments and their constituent proteins. Experimental Cell Research, 313(10):2236-2243, 2007.

[56] K.J. Green and C.A. Gaudry. Are desmosomes more than tethers for intermediate filaments? Nature Reviews Molecular Cell Biology, 1(3):208-216, 2000.

[57] L. Borradori and A. Sonnenberg. Structure and function of hemidesmosomes: More than simple adhesion complexes. Journal of Investigative Dermatology, 112(4):411-418, 1999.

[58] K. Wilhelmsen, S.H.M. Litjens, I. Kuikman, N. Tshimbalanga, H. Janssen, I. Van Den Bout, K. Raymond, and A. Sonnenberg. Nesprin-3, a novel outer nuclear membrane protein, associates with the cytoskeletal linker protein plectin. The Journal of Cell Biology, 171(5):799-810, 2005.

[59] X. Pan, R.P. Hobbs, and P.A. Coulombe. The expanding significance of keratin intermediate filaments in normal and diseased epithelia. Current Opinion in Cell Biology, 25(1):47-56, 2013.

[60] P. Vijayaraj, C. Kröger, U. Reuter, R. Windoffer, R.E. Leube, and T.M. Magin. Keratins regulate protein biosynthesis through localization of GLUT1 and-3 upstream of AMP kinase and raptor. The Journal of Cell Biology, 187(2):175-184, 2009.

[61] M. Beil, A. Micoulet, G. von Wichert, S. Paschke, P. Walther, M.B. Omary, P.P. Van Veldhoven, U. Gern, E. Wolff-Hieber, J. Eggermann, et al. Sphingosylphosphorylcholine regulates keratin network architecture and visco-elastic properties of human cancer cells. Nature Cell Biology, 5(9):803-811, 2003.

[62] M.B. Omary, P.A. Coulombe, and W.H.I. McLean. Intermediate filament proteins and their associated diseases. New England Journal of Medicine, 351(20):2087-2100, 2004. 
[63] E. Fuchs and D.W. Cleveland. A structural scaffolding of intermediate filaments in health and disease. Science, 279(5350):514-519, 1998.

[64] V. Karantza. Keratins in health and cancer: More than mere epithelial cell markers. Oncogene, 30(2):127-138, 2011.

[65] J. Uitto, G. Richard, and J.A. McGrath. Diseases of epidermal keratins and their linker proteins. Experimental Cell Research, 313(10):1995-2009, 2007.

[66] Y. Chan, I. Anton-Lamprecht, Q.C. Yu, A. Jäckel, B. Zabel, J.P. Ernst, and E. Fuchs. A human keratin 14 "knockout": The absence of K14 leads to severe epidermolysis bullosa simplex and a function for an intermediate filament protein. Genes \& Development, 8(21):2574-2587, 1994.

[67] M.B. Omary, N.O. Ku, P. Strnad, and S. Hanada. Toward unraveling the complexity of simple epithelial keratins in human disease. The Journal of Clinical Investigation, 119(7):1794, 2009.

[68] P. Strnad, Q. Zhou, S. Hanada, L.C. Lazzeroni, B.H. Zhong, P. So, T.J. Davern, W.M. Lee, and M.B. Omary. Keratin variants predispose to acute liver failure and adverse outcome: Race and ethnic associations. Gastroenterology, 139(3):828-835, 2010.

[69] K.M. Ridge, L. Linz, F.W. Flitney, E.R. Kuczmarski, Y.H. Chou, M.B. Omary, J.I. Sznajder, and R.D. Goldman. Keratin 8 phosphorylation by protein kinase $\mathrm{C} \delta$ regulates shear stress-mediated disassembly of keratin intermediate filaments in alveolar epithelial cells. Journal of Biological Chemistry, 280(34):30400-30405, 2005.

[70] A. Jaitovich, S. Mehta, N. Na, A. Ciechanover, R.D. Goldman, and K.M. Ridge. Ubiquitin-proteasome-mediated degradation of keratin intermediate filaments in mechanically stimulated A549 cells. Journal of Biological Chemistry, 283(37):25348-25355, 2008.

[71] S. Sivaramakrishnan, J.V. DeGiulio, L. Lorand, R.D. Goldman, and K.M. Ridge. Micromechanical properties of keratin intermediate filament networks. Proceedings of the National Academy of Sciences, 105(3):889-894, 2008.

[72] S. Sivaramakrishnan, J.L. Schneider, A. Sitikov, R.D. Goldman, and K.M. Ridge. Shear stress induced reorganization of the keratin intermediate filament network requires phosphorylation by protein kinase $\mathrm{C} \zeta$. Molecular Biology of the Cell, 20(11):2755-2765, 2009. 
[73] C.P. Brangwynne, F.C. MacKintosh, S. Kumar, N.A. Geisse, J. Talbot, L. Mahadevan, K.K. Parker, D.E. Ingber, and D.A. Weitz. Microtubules can bear enhanced compressive loads in living cells because of lateral reinforcement. The Journal of Cell Biology, 173(5):733-741, 2006.

[74] S. Kaech, B. Ludin, and A. Matus. Cytoskeletal plasticity in cells expressing neuronal microtubule-associated proteins. Neuron, 17(6):1189-1199, 1996.

[75] N. Wang, K. Naruse, D. Stamenović, J.J. Fredberg, S.M. Mijailovich, I.M. Tolić-Nørrelykke, T. Polte, R. Mannix, and D.E. Ingber. Mechanical behavior in living cells consistent with the tensegrity model. Proceedings of the National Academy of Sciences, 98(14):7765-7770, 2001.

[76] A.W. Schaefer, N. Kabir, and P. Forscher. Filopodia and actin arcs guide the assembly and transport of two populations of microtubules with unique dynamic parameters in neuronal growth cones. The Journal of Cell Biology, 158(1):139-152, 2002.

[77] C.P. Brangwynne, G.H. Koenderink, F.C. MacKintosh, and D.A. Weitz. Nonequilibrium microtubule fluctuations in a model cytoskeleton. Physical Review Letters, 100(11):118104, 2008.

[78] L. Lu, Y. Feng, W.J. Hucker, S.J. Oswald, G.D. Longmore, and F.C.P. Yin. Actin stress fiber pre-extension in human aortic endothelial cells. Cell Motility and the Cytoskeleton, 65(4):281-294, 2008.

[79] M Das, A.J. Levine, and F.C. MacKintosh. Buckling and force propagation along intracellular microtubules. Europhysics Letters, 84(1):18003, 2008.

[80] W.L. Shan, Z. Chen, C.P. Broedersz, A.A. Gumaste, W.O. Soboyejo, and C.P. Brangwynne. Attenuated short wavelength buckling and force propagation in a biopolymer-reinforced rod. Soft Matter, 9(1):194-199, 2013.

[81] H. Jiang and J. Zhang. Mechanics of microtubule buckling supported by cytoplasm. Journal of Applied Mechanics, 75(6):061019, 2008.

[82] T. Li. A mechanics model of microtubule buckling in living cells. Journal of Biomechanics, 41(8):1722-1729, 2008.

[83] A. Leibovitz, W.M. McCombs 3rd, D. Johnston, C.E. McCoy, and J.C. Stinson. New human cancer cell culture lines. I. SW-13, small-cell carcinoma of the adrenal cortex. Journal of the National Cancer Institute, 51(2):691, 1973. 
[84] P. Strnad, R. Windoffer, and R.E. Leube. Induction of rapid and reversible cytokeratin filament network remodeling by inhibition of tyrosine phosphatases. Journal of Cell Science, 115(21):4133-4148, 2002.

[85] C. Gerthsen and D. Meschede. Gerthsen Physik. Springer-Lehrbuch. Springer, 22 edition, 2003.

[86] J.R. Lakowicz. Principles of Fluorescence Spectroscopy. Springer, 2007.

[87] data sheet olympus; effective 25.02.2014. http://www.olympusamerica.com/ seg_section/uis2/seg_uis2_mr_umniba3.asp.

[88] Fluorescence SpectraViewer; effective 25.02.2014. http:// www.lifetechnologies.com/de/de/home/life-science/cell-analysis/ labeling-chemistry/fluorescence-spectraviewer.html.

[89] J.C. McDonald and G.M. Whitesides. Poly (dimethylsiloxane) as a material for fabricating microfluidic devices. Accounts of Chemical Research, 35(7):491499, 2002.

[90] D.C. Duffy, J.C. McDonald, O.J.A. Schueller, and G.M. Whitesides. Rapid prototyping of microfluidic systems in poly (dimethylsiloxane). Analytical Chemistry, 70(23):4974-4984, 1998.

[91] Y. Xia and G.M. Whitesides. Soft lithography. Annual Review of Materials Science, 28(1):153-184, 1998.

[92] data sheet microchem; effective 03.02.2014. http://microchem.com/pdf/SU$8 \% 203000 \% 20$ Data $\% 20$ Sheet.pdf.

[93] J.F. Nolting and S. Köster. Influence of microfluidic shear on keratin networks in living cells. New Journal of Physics, 15(4):045025, 2013.

[94] H. Bruus. Theoretical microfluidics, volume 18. Oxford University Press, 2008.

[95] E.M. Purcell. Life at low reynolds number. American Journal of Physics, 45(1):3-11, 1977.

[96] J.F. Nolting. Influence of Microfluidic Shear on Keratin Networks in Live Cells. Diplomathesis, Georg-August-Universität Göttingen, 2010.

[97] M. Guizar-Sicairos, S.T. Thurman, and J.R. Fienup. Efficient subpixel image registration algorithms. Optics Letters, 33(2):156-158, 2008. 
[98] I.Y. Wong, M.L. Gardel, D.R. Reichman, E.R. Weeks, M.T. Valentine, A.R. Bausch, and D.A. Weitz. Anomalous diffusion probes microstructure dynamics of entangled F-actin networks. Physical Review Letters, 92(17):178101, 2004.

[99] L. Bruno, V. Levi, M. Brunstein, and M.A. Despósito. Transition to superdiffusive behavior in intracellular actin-based transport mediated by molecular motors. Physical Review E, 80(1):011912, 2009.

[100] H.F. Lodish, A. Berk, S.L. Zipursky, P. Matsudaira, D. Baltimore, and J. Darnell. Molecular cell biology. WH Freeman New York, 2000.

[101] A.F. Straight, A. Cheung, J. Limouze, I. Chen, N.J. Westwood, J.R. Sellers, and T.J. Mitchison. Dissecting temporal and spatial control of cytokinesis with a myosin II inhibitor. Science, 299(5613):1743-1747, 2003.

[102] M. Kovács, J. Tóth, C. Hetényi, A. Málnási-Csizmadia, and J.R. Sellers. Mechanism of blebbistatin inhibition of myosin II. Journal of Biological Chemistry, 279(34):35557-35563, 2004.

[103] J. Kolega. Phototoxicity and photoinactivation of blebbistatin in UV and visible light. Biochemical and Biophysical Research Communications, 320(3):1020-1025, 2004.

[104] R.A.L. Jones. Soft condensed matter, volume 6. Oxford University Press, 2002.

[105] N. Walter, T. Busch, T. Seufferlein, and J.P. Spatz. Elastic moduli of living epithelial pancreatic cancer cells and their skeletonized keratin intermediate filament network. Biointerphases, 6(2):79-85, 2011.

[106] A.J. Ridley, M.A. Schwartz, K. Burridge, R.A. Firtel, M.H. Ginsberg, G. Borisy, J.T. Parsons, and A.R. Horwitz. Cell migration: Integrating signals from front to back. Science, 302(5651):1704-1709, 2003.

[107] K. Woo, A.K. Dutta, V. Patel, C. Kresge, and A.P. Feranchak. Fluid flow induces mechanosensitive ATP release, calcium signalling and Cl- transport in biliary epithelial cells through a PKC $\zeta$-dependent pathway. The Journal of Physiology, 586(11):2779-2798, 2008.

[108] P. Bodin and G. Burnstock. Evidence that release of adenosine triphosphate from endothelial cells during increased shear stress is vesicular. Journal of Cardiovascular Pharmacology, 38(6):900-908, 2001. 
[109] A.I. Masyuk, T.V. Masyuk, P.L. Splinter, B.Q. Huang, A.J. Stroope, and N.F. LaRusso. Cholangiocyte cilia detect changes in luminal fluid flow and transmit them into intracellular Ca2+ and cAMP signaling. Gastroenterology, 131(3):911-920, 2006.

[110] K. Riento and A.J. Ridley. Rocks: Multifunctional kinases in cell behaviour. Nature Reviews Molecular Cell Biology, 4(6):446-456, 2003.

[111] G. Totsukawa, Y. Yamakita, S. Yamashiro, D.J. Hartshorne, Y. Sasaki, and F. Matsumura. Distinct roles of ROCK (Rho-kinase) and MLCK in spatial regulation of MLC phosphorylation for assembly of stress fibers and focal adhesions in 3T3 fibroblasts. The Journal of Cell Biology, 150(4):797-806, 2000.

[112] R.P. Franke, M. Gräfe, H. Schnittler, D. Seiffge, C. Mittermayer, and D. Drenckhahn. Induction of human vascular endothelial stress fibres by fluid shear stress. Nature, 307:648-649, 1984.

[113] A.M. Malek and S. Izumo. Mechanism of endothelial cell shape change and cytoskeletal remodeling in response to fluid shear stress. Journal of Cell Science, 109(4):713-726, 1996.

[114] D.E. Discher, P. Janmey, and Y.I. Wang. Tissue cells feel and respond to the stiffness of their substrate. Science, 310(5751):1139-1143, 2005.

[115] T. Yeung, P.C. Georges, L.A. Flanagan, B. Marg, M. Ortiz, M. Funaki, N. Zahir, W. Ming, V. Weaver, and P.A. Janmey. Effects of substrate stiffness on cell morphology, cytoskeletal structure, and adhesion. Cell Motility and the Cytoskeleton, 60(1):24-34, 2005.

[116] J. Solon, I. Levental, K. Sengupta, P.C. Georges, and P.A. Janmey. Fibroblast adaptation and stiffness matching to soft elastic substrates. Biophysical Journal, 93(12):4453-4461, 2007.

[117] S. Timoshenko. Theory of elastic stability. Engineering societies monographs. McGraw-Hill, 1961.

[118] J. Howard. Mechanics of Motor Proteins and the Cytoskeleton. Sinauer Associates, Incorporated, 2001.

[119] L.D. Landau and E.M. Lifschitz. Band VII Elastizitätstheorie. Akademie Verlag, Berlin, 1989. 
[120] M.B. Amar, A. Goriely, M.M. Müller, and L. Cugliandolo. New Trends in the Physics and Mechanics of Biological Systems: Lecture Notes of the Les Houches Summer School: Volume 92, July 2009. Lecture Notes of the Les Houches Summer School. OUP Oxford, 2011.

[121] M.M. Tirado and J.G. de La Torre. Translational friction coefficients of rigid, symmetric top macromolecules. Application to circular cylinders. The Journal of Chemical Physics, 71(6):2581-2587, 1979.

[122] Bronštejn, Semendjajew, Musiol, and Mühlig. Taschenbuch der Mathematik, volume 5. Springer, 1993.

[123] J. Howard and J.F. Ashmore. Stiffness of sensory hair bundles in the sacculus of the frog. Hearing Research, 23(1):93-104, 1986.

[124] N. Otsu. A threshold selection method from gray-level histograms. Automatica, 11(285-296):23-27, 1975.

[125] L. Lam, S.W. Lee, and C.Y. Suen. Thinning methodologies - A comprehensive survey. IEEE Transactions on Pattern Analysis and Machine Intelligence, 14(9):869-885, 1992.

[126] E. Hecht. Optik. Oldenbourg, 2009.

[127] W.H. Richardson. Bayesian-based iterative method of image restoration. JOSA, 62(1):55-59, 1972.

[128] L.B. Lucy. An iterative technique for the rectification of observed distributions. The Astronomical Journal, 79:745, 1974.

[129] J. Pawley. Handbook of biological confocal microscopy. Springer, 2010.

[130] Matlab exchange script by T. Peng; effective August 2013. http: //www.mathworks.de/matlabcentral/fileexchange/9168-detectcircles-with-various-radii-in-grayscale-image-via-houghtransform/content/CircularHough_Grd.m.

[131] L. Norlén and A. Al-Amoudi. Stratum corneum keratin structure, function, and formation: The cubic rod-packing and membrane templating model. Journal of Investigative Dermatology, 123(4):715-732, 2004.

[132] L. Norlen. Stratum corneum keratin structure, function and formation - A comprehensive review. International Journal of Cosmetic Science, 28(6):397425, 2006 . 
[133] J. Alcaraz, L. Buscemi, M. Grabulosa, X. Trepat, B. Fabry, R. Farré, and D. Navajas. Microrheology of human lung epithelial cells measured by atomic force microscopy. Biophysical Journal, 84(3):2071-2079, 2003.

[134] B. Nöding and S. Köster. Intermediate filaments in small configuration spaces. Physical Review Letters, 108(8):088101, 2012.

[135] Jan Rother. Personal communication. 102013.

[136] F. G Schmidt, F. Ziemann, and E. Sackmann. Shear field mapping in actin networks by using magnetic tweezers. European Biophysics Journal, 24(5):348353, 1996.

[137] F. Gittes, B. Mickey, J. Nettleton, and J. Howard. Flexural rigidity of microtubules and actin filaments measured from thermal fluctuations in shape. The Journal of Cell Biology, 120(4):923-934, 1993.

[138] E.S. Reynolds. The use of lead citrate at high $\mathrm{pH}$ as an electron-opaque stain in electron microscopy. The Journal of Cell Biology, 17(1):208-212, 1963. 


\section{List of Publications}

- J.-F. Nolting and S. Köster.

Influence of microfluidic shear on keratin networks in living cells.

New Journal of Physics, 15, 045025, (2013).

- B. Weinhausen, J.-F. Nolting, C. Olendrowitz, J. Langfahl-Klabes, M. Reynolds, T. Salditt and S. Köster.

X-ray nano-diffraction on cytoskeletal networks.

New Journal of Physics, 14, 085013, (2012).

- M. E. Brennich, J.-F. Nolting, C. Dammann, B. Nöding, S. Bauch, H. Herrmann, T. Pfohl and S. Köster.

Dynamics of intermediate filament assembly followed in micro-flow by small angle x-ray scattering.

Lab on a Chip 11, 708-716, (2011). 


\section{Lebenslauf}

\section{Jens-Friedrich Nolting}

geboren am 03.05.1985 in Hannover, Deutschland

\section{Schulischer und wissenschaftlicher Werdegang}

seit $11 / 2010$

$10 / 2010$

$10 / 2005-10 / 2010$

$06 / 2005$
Promotion im Rahmen der „Göttingen Graduate School for Neurosciences, Biophysics, and Molecular Biosciences (GGNB)“. Wissenschaftlicher Mitarbeiter am „Courant Research Centre“ und am Institut für Röntgenphysik an der Georg-August-Universität Göttingen.

Erlangung des Grades Diplom-Physiker nach Abschluss der Diplomarbeit mit dem Titel „Influence of Microfluidic Shear on Keratin Networks in Live Cells" am „Courant Research Centre" an der Georg-AugustUniversität Göttingen.

Diplomstudiengang Physik an der Georg-AugustUniversität Göttingen

Erlangung der allgemeinen Hochschulreife am Gymnasium Isernhagen 


\section{Danksagung}

Abschließend möchte ich mich bei allen Menschen herzlich bedanken, die zum Gelingen meiner Doktorarbeit beigetragen haben.

An erster Stelle möchte ich Prof. Dr. Sarah Köster danken. Neben der Möglichkeit meine Arbeit bei ihr in der Arbeitsgruppe anzufertigen, gilt ihr auch mein Dank für ihre umfassende Diskussionsbereitschaft und für die Ermöglichung von Besuchen zahlreicher Konferenzen.

Prof. Dr. Andreas Janshoff danke ich sehr für die Übernahme des Koreferats meiner Dissertation und für sein mir entgegengebrachtes Interesse und Engagement bezüglich meiner Arbeit als Mitglied meines Betreuungsausschusses.

Des Weiteren möchte ich mich bei Prof. Dr. Tim Salditt für inspirierende und motivierende Diskussionen im und außerhalb des Rahmens meines Thesis Committees herzlich bedanken.

Bei Dr. Florian Rehfeldt, Ph.D. Thomas Burg und Prof. Dr. Jörg Enderlein möchte ich mich für ihr Interesse und ihre Zeit bedanken, meinem Prüfungskomitee beizuwohnen.

Darüber hinaus gilt mein Dank Prof. Dr. Rudolf Leube, Dr. Dr. Reinhard Windoffer und Dr. Anne Kölsch für die Bereitstellung der Zelllinie und für Diskussionen.

Dr. Wiebke Möbius möchte ich für ihren Einsatz bei der Aufnahme von TEM Bildern von Keratinbündeln in Zellen danken.

Zusätzlich danke ich Florian Schlosser und Dr. Florian Rehfeldt für die Ermöglichung der Transfektionsexperimente.

Jan Rother und Prof. Dr. Andreas Janshoff danke ich für die Weitergabe und Diskussion ihrer AFM-Messungen an den SK8/18-2 Zellen.

Für eine tolle Arbeitsatmosphäre und eine unvergessliche Zeit möchte ich mich bei den Menschen des gesamten Instituts für Röntgenphysik bedanken. Insbesondere 
gilt hierbei mein Dank meinen Bürokollegen Susanne Bauch, Christian Dammann und Bernd Nöding für ein großartiges Umfeld mit sowohl unterhaltsamen als auch zielführenden Diskussionen. Auch danke ich Dr. Britta Weinhausen und Jannick Langfahl-Klabes für die Unterstützung bei Zellkulturarbeiten und ergiebige Diskussionen. Des Weiteren möchte ich mich bei Joachim Herbst und Jan Goeman bedanken, die in technischen Belangen immer eine schnelle und hilfsbereite Antwort hatten.

Für das Korrekturlesen dieser Arbeit danke ich zudem Bernd Nöding, Christian Dammann, Oliva Saldanha, Rabea Sandmann und Viktor Schroeder.

Persönlich möchte ich mich auch bei Andreas, Benedikt, Bashkim, Dominik, Matthias, Matthias und Philipp für eine prägende und unvergessliche Zeit in Göttingen bedanken.

Meinen Eltern Brunhild und Friedrich Nolting gilt mein ganz besonderer Dank. Ihre Unterstützung während meines Studiums und der Promotion hat mir stets viel bedeutet.

Schlussendlich danke ich meiner Freundin Sarah Schwarz von ganzem Herzen für ihre grenzenlose und unentwegt liebevolle Unterstützung. 